\title{
TRAVELING WAVES FOR SPATIALLY DISCRETE SYSTEMS OF FITZHUGH-NAGUMO TYPE WITH PERIODIC COEFFICIENTS*
}

\author{
WILLEM M. SCHOUTEN-STRAATMAN ${ }^{\dagger}$ AND HERMEN JAN HUPKES ${ }^{\ddagger}$
}

\begin{abstract}
We establish the existence and nonlinear stability of traveling wave solutions for a class of lattice differential equations (LDEs) that includes the discrete FitzHugh-Nagumo system with alternating scale-separated diffusion coefficients. In particular, we view such systems as singular perturbations of spatially homogeneous LDEs, for which stable traveling wave solutions are known to exist in various settings. The two-periodic waves considered in this paper are described by singularly perturbed multicomponent functional differential equations of mixed type (MFDEs). In order to analyze these equations, we generalize the spectral convergence technique that was developed by Bates, Chen, and Chmaj to analyze the scalar Nagumo LDE. This allows us to transfer several crucial Fredholm properties from the spatially homogeneous to the spatially periodic setting. Our results hence do not require the use of comparison principles or exponential dichotomies.
\end{abstract}

Key words. lattice differential equations, FitzHugh-Nagumo system, periodic coefficients, singular perturbation

AMS subject classifications. 34A33, 34K08, 34K26, 34K 31

DOI. $10.1137 / 18 \mathrm{M} 1204942$

1. Introduction. In this paper we consider a class of lattice differential equations (LDEs) that includes the FitzHugh-Nagumo system

$$
\begin{aligned}
\dot{u}_{j} & =d_{j}\left(u_{j+1}+u_{j-1}-2 u_{j}\right)+g\left(u_{j} ; a_{j}\right)-w_{j}, \\
\dot{w}_{j} & =\rho_{j}\left[u_{j}-\gamma_{j} w_{j}\right]
\end{aligned}
$$

with cubic nonlinearities

$$
g(u ; a)=u(1-u)(u-a)
$$

and two-periodic coefficients

$$
(0, \infty) \times(0,1) \times(0,1) \times(0, \infty) \ni\left(d_{j}, a_{j}, \rho_{j}, \gamma_{j}\right)=\left\{\begin{array}{r}
\left(\varepsilon^{-2}, a_{o}, \rho_{o}, \gamma_{o}\right) \text { for odd } j \\
\left(1, a_{e}, \rho_{e}, \gamma_{e}\right) \text { for even } j .
\end{array}\right.
$$

We assume that the diffusion coefficients are of different orders in the sense $0<\varepsilon \ll 1$. Building on the results obtained in $[29,30]$ for the spatially homogeneous FitzHughNagumo LDE, we show that (1.1) admits stable traveling pulse solutions with separate waveprofiles for the even and odd lattice sites. The main ingredient in our approach is a spectral convergence argument, which allows us to transfer Fredholm properties between linear operators acting on different spaces.

*Received by the editors August 2, 2018; accepted for publication (in revised form) July 1, 2019; published electronically August 21, 2019.

https://doi.org/10.1137/18M1204942

Funding: The work of the authors was supported by the Netherlands Organization for Scientific Research (NWO) grant 639.032.612.

$\dagger^{\dagger}$ Corresponding author. Mathematisch Instituut-Universiteit Leiden, 2300 RA Leiden, The Netherlands (w.m.schouten@math.leidenuniv.nl).

${ }^{\ddagger}$ Mathematisch Instituut-Universiteit Leiden, 2300 RA Leiden, The Netherlands (hhupkes@ math.leidenuniv.nl). 
Signal propagation. The LDE (1.1) can be interpreted as a spatially inhomogeneous discretization of the FitzHugh-Nagumo partial differential equation (PDE)

$$
\begin{aligned}
u_{t} & =u_{x x}+g(u ; a)-w, \\
w_{t} & =\rho[u-\gamma w],
\end{aligned}
$$

again with $\rho>0$ and $\gamma>0$. This PDE was proposed in the 1960s [21, 22] as a simplification of the four-component system that Hodgkin and Huxley developed to describe the propagation of spike signals through the nerve fibers of giant squids [26]. Indeed, for small $\rho>0(1.4)$ admits isolated pulse solutions of the form

$$
(u, w)(x, t)=\left(\bar{u}_{0}, \bar{w}_{0}\right)\left(x+c_{0} t\right),
$$

in which $c_{0}$ is the wavespeed and the waveprofile $\left(\bar{u}_{0}, \bar{w}_{0}\right)$ satisfies the limits

$$
\lim _{|\xi| \rightarrow \infty}\left(\bar{u}_{0}, \bar{w}_{0}\right)(\xi)=0
$$

Such solutions were first observed numerically by FitzHugh [23], but the rigorous analysis of these pulses turned out to be a major mathematical challenge that is still ongoing. Many techniques have been developed to obtain the existence and stability of such pulse solutions in various settings, including geometric singular perturbation theory $[8,25,34,33]$, Lin's method [36, 10, 9], the variational principle [11], and the Maslov index $[13,14]$.

It turns out that electrical signals can only reach feasible speeds when traveling through nerve fibers that are insulated by a myelin coating. Such coatings are known to admit regularly spaced gaps at the nodes of Ranvier [41], where propagating signals can be chemically reinforced. In fact, the action potentials effectively jump from one node to the next through a process caused saltatory conduction [37]. In order to include these effects, it is natural [35] to replace (1.4) by the FitzHugh-Nagumo LDE

$$
\begin{aligned}
\dot{u}_{j} & =\frac{1}{\varepsilon^{2}}\left(u_{j+1}+u_{j-1}-2 u_{j}\right)+g\left(u_{j} ; a\right)-w_{j}, \\
\dot{w}_{j} & =\rho\left[u_{j}-\gamma w_{j}\right] .
\end{aligned}
$$

In this equation the variable $u_{j}$ describes the potential at the node $j \in \mathbb{Z}$ node, while $w_{j}$ describes the dynamics of the recovery variables. We remark that this LDE arises directly from (1.4) by using the nearest-neighbor discretization of the Laplacian on a grid with spacing $\varepsilon>0$.

In [30, 29], Hupkes and Sandstede studied (1.7) and showed that for $a$ sufficiently far from $\frac{1}{2}$ and small $\rho>0$, there exists a stable locally unique traveling pulse solution

$$
\left(u_{j}, w_{j}\right)(t)=(\bar{u}, \bar{w})(j+c t) .
$$

The techniques relied on exponential dichotomies and Lin's method to develop an infinite-dimensional analogue of the exchange lemma. In [20] the existence part of these results was generalized to versions of (1.7) that feature infinite-range discretizations of the Laplacian that involve all neighbors instead of only the nearest-neighbors. The stability results were also recently generalized to this setting [44], but only for small $\varepsilon>0$ at present. Such systems with infinite-range interactions play an important role in neural field models $[4,3,40,45]$, which aim to describe the dynamics of large networks of neurons. 


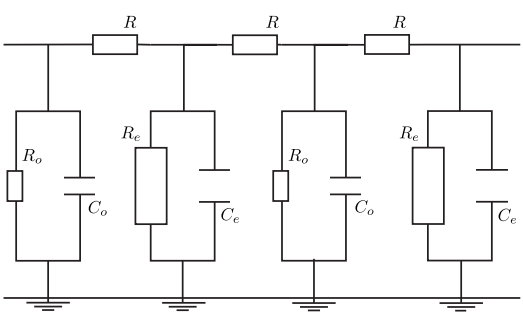

(a)

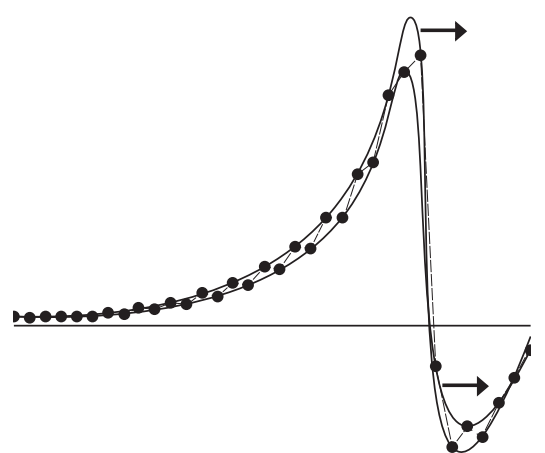

(b)

FIG. 1.1. (a) Simplified representation of the system (1.1) as an electrical circuit in a nerve fiber, analogous to [4, Fig. 1.11]. In this paper, the resistances $R_{o}$ and $R_{e}$, as well as the capacitances $C_{o}$ and $C_{e}$ in the cell membrane, alternate between the even and odd membranes. The resistivity of the intracellular fluid $R$ is constant. (b) Schematic representation of the $u$-component of a traveling pulse for the system (1.1), which alternates between two waveprofiles.

Our motivation here for studying the 2-periodic version (1.1) of the FitzHughNagumo LDE (1.7) comes from recent developments in optical nanoscopy. Indeed, the results in $[49,15,16]$ clearly show that certain proteins in the cytoskeleton of nerve fibers are organized periodically. This periodicity turns out to be a universal feature of all nerve systems, not just those which are insulated with a myelin coating. Since it also manifests itself at the nodes of Ranvier, it is natural to allow the parameters in (1.7) to vary in a periodic fashion. This can be understood by considering the generic circuit-models that are typically used to model nerve axons; see Figure 1.1(a).

The results in this paper are a first step in this direction. The restriction on the diffusion parameters is rather severe, but the absence of a comparison principle forces us to take a perturbative approach. We emphasize that the scale separation in the diffusion coefficients means that there is no natural continuum limit for (1.9) that can be recovered by sending the node separation to zero.

Periodicity. Periodic patterns are frequently encountered when studying the behavior of physical systems that have a discrete underlying spatial structure. Examples include the presence of twinning microstructures in shape memory alloys [2] and the formation of domain-wall microstructures in dielectric crystals [46].

At present, however, the mathematical analysis of such models has predominantly focused on one-component systems. For example, the results in [12] cover the bistable Nagumo LDE

$$
\dot{u}_{j}=d_{j}\left(u_{j+1}+u_{j-1}-2 u_{j}\right)+g\left(u_{j} ; a_{j}\right)
$$

with spatially periodic coefficients $\left(d_{j}, a_{j}\right) \in(0, \infty) \times(0,1)$. Exploiting the comparison principle, the authors were able to establish the existence of stable traveling wave solutions. Similar results were obtained in [24] for monostable versions of (1.9).

Let us also mention the results in $[18,19,27]$, where the authors consider chains of alternating masses connected by identical springs (and vice versa). The dynamical behavior of such systems can be modeled by LDEs of Fermi-Pasta-Ulam type with periodic coefficients. In certain limiting cases the authors were able to construct so-called nanopterons, which are multicomponent wave solutions that have low-amplitude oscillations in their tails. 
In the examples above the underlying periodicity is built into the spatial system itself. However, periodic patterns also arise naturally as solutions to spatially homogeneous discrete systems. As an example, systems of the form (1.9) with homogeneous but negative diffusion coefficients $d_{j}=d<0$ have been used to describe phase transitions for grids of particles that have visco-elastic interactions [6, 7, 47]. Upon introducing separate scalings for the odd and even lattice sites, this one-component LDE can be turned into a 2-periodic system of the form

$$
\begin{aligned}
\dot{v}_{j} & =d_{e}\left(w_{j}+w_{j-1}-2 v_{j}\right)-f_{e}\left(v_{j}\right), \\
\dot{w}_{j} & =d_{o}\left(v_{j+1}+v_{j}-2 w_{j}\right)-f_{o}\left(w_{j}\right)
\end{aligned}
$$

with positive coefficients $d_{e}>0$ and $d_{o}>0$. Systems of this type have been analyzed in considerable detail in $[5,48]$, where the authors establish the co-existence of patterns that can be both monostable and bistable in nature.

As a final example, let us mention that the LDE (1.9) with positive spatially homogeneous diffusion coefficients $d_{j}=d>0$ can admit many periodic equilibria [38]. In [28] the authors construct bichromatic traveling waves that connect spatially homogeneous rest-states with such 2-periodic equilibria. Such waves can actually travel in parameter regimes where the standard monochromatic waves that connect zero to one are trapped. This presents a secondary mechanism by which the stable states zero and one can spread throughout the spatial domain.

Wave equations. Returning to the 2-periodic FitzHugh-Nagumo LDE (1.1), we use the traveling wave Ansatz

$$
(u, w)_{j}(t)=\left\{\begin{array}{l}
\left(\bar{u}_{o}, \bar{w}_{o}\right)(j+c t) \text { when } j \text { is odd } \\
\left(\bar{u}_{e}, \bar{w}_{e}\right)(j+c t) \text { when } j \text { is even }
\end{array}\right.
$$

illustrated in Figure 1.1(b) to arrive at the coupled system

$$
\begin{aligned}
c \bar{u}_{o}^{\prime}(\xi) & =\frac{1}{\varepsilon^{2}}\left(\bar{u}_{e}(\xi+1)+\bar{u}_{e}(\xi-1)-2 \bar{u}_{o}(\xi)\right)+g\left(\bar{u}_{o}(\xi) ; a_{o}\right)-\bar{w}_{o}(\xi), \\
c \bar{w}_{o}^{\prime}(\xi) & =\rho_{o}\left[\bar{u}_{o}(\xi)-\gamma_{o} \bar{w}_{o}(\xi)\right], \\
c \bar{u}_{e}^{\prime}(\xi) & =\left(\bar{u}_{o}(\xi+1)+\bar{u}_{o}(\xi-1)-2 \bar{u}_{e}(\xi)\right)+g\left(\bar{u}_{e}(\xi) ; a_{e}\right)-\bar{w}_{e}(\xi), \\
c \bar{w}_{e}^{\prime}(\xi) & =\rho_{e}\left[\bar{u}_{e}(\xi)-\gamma_{e} \bar{w}_{e}(\xi)\right] .
\end{aligned}
$$

Multiplying the first line by $\varepsilon^{2}$ and then taking $\varepsilon \downarrow 0$, we obtain the direct relation

$$
\bar{u}_{o}(\xi)=\frac{1}{2}\left[\bar{u}_{e}(\xi+1)+\bar{u}_{e}(\xi-1)\right],
$$

which can be substituted into the last two lines to yield

$$
\begin{aligned}
c \bar{u}_{e}^{\prime}(\xi) & =\frac{1}{2}\left(\bar{u}_{e}(\xi+2)+\bar{u}_{e}(\xi-2)-2 \bar{u}_{e}(\xi)\right)+g\left(\bar{u}_{e}(\xi) ; a_{e}\right)-\bar{w}_{e}(\xi), \\
c \bar{w}_{e}^{\prime}(\xi) & =\rho_{e}\left[\bar{u}_{e}(\xi)-\gamma_{e} \bar{w}_{e}(\xi)\right] .
\end{aligned}
$$

All the odd variables have been eliminated from this last equation, which in fact describes pulse solutions to the spatially homogeneous FitzHugh-Nagumo LDE (1.7). Plugging these pulses into the remaining equation we arrive at

$$
c \bar{w}_{o}^{\prime}(\xi)+\rho_{o} \gamma_{o} \bar{w}_{o}(\xi)=\frac{1}{2} \rho_{o}\left[\bar{u}_{e}(\xi+1)+\bar{u}_{e}(\xi-1)\right] .
$$


This can be solved to yield the remaining second component of a singular pulse solution that we denote by

$$
\bar{U}_{0}=\left(\bar{u}_{o ; 0}, \bar{w}_{o ; 0}, \bar{u}_{e ; 0}, \bar{w}_{e ; 0}\right) .
$$

The main task in this paper is to construct stable traveling wave solutions to (1.1) by continuing this singular pulse into the regime $0<\varepsilon \ll 1$. We use a functional analytic approach to handle this singular perturbation, focusing on the linear operator associated to the linearization of (1.12) with $\varepsilon>0$ around the singular pulse. We show that this operator inherits several crucial Fredholm properties that were established in [30] for the linearization of (1.14) around the even pulse $\left(\bar{u}_{e ; 0}, \bar{w}_{e ; 0}\right)$.

Our results are not limited to the two-component system (1.1). Indeed, we consider general $(n+k)$-dimensional reaction diffusion systems with 2-periodic coefficients, where $n \geq 1$ is the number of components with a nonzero diffusion term and $k \geq 0$ is the number of components that do not diffuse. We can handle both traveling fronts and traveling pulses, but do impose conditions on the end-states that are stronger than the usual temporal stability requirements. Indeed, at times we will require (submatrices of) the corresponding Jacobians to be negative definite instead of merely spectrally stable. We emphasize that these distinctions disappear for scalar problems. In particular, our framework also covers the Nagumo LDE (1.9), but does not involve the use of a comparison principle.

Spectral convergence. The main inspiration for our approach is the spectral convergence technique that was developed in [1] to establish the existence of traveling wave solutions to the homogeneous Nagumo $\operatorname{LDE}^{1}$ (1.9) with diffusion coefficients $d_{j}=1 / \varepsilon^{2} \gg 1$. The linear operator

$$
\mathcal{L}_{\varepsilon} v(\xi)=c_{0} v^{\prime}(\xi)-\frac{1}{\varepsilon^{2}}[v(\xi+\varepsilon)+v(\xi-\varepsilon)-2 v(\xi)]-g_{u}\left(\bar{u}_{0}(\xi) ; a\right) v(\xi)
$$

plays a crucial role in this approach, where the pair $\left(c_{0}, \bar{u}_{0}\right)$ is the traveling front solution of the Nagumo PDE

$$
u_{t}=u_{x x}+g(u ; a)
$$

This front solutions satisfies the system

$$
c_{0} \bar{u}_{0}^{\prime}(\xi)=\bar{u}_{0}^{\prime \prime}(\xi)+g(\bar{u}(\xi) ; a), \quad \bar{u}_{0}(-\infty)=0, \quad \bar{u}_{0}(+\infty)=1,
$$

to which we can associate the linear operator

$$
\left[\mathcal{L}_{0} v\right](\xi)=c_{0} v^{\prime}(\xi)-v^{\prime \prime}(\xi)-g_{u}(\bar{u}(\xi) ; a) v(\xi),
$$

which can be interpreted as the formal $\varepsilon \downarrow 0$ limit of (1.17). It is well-known that $\mathcal{L}_{0}+\delta: H^{2} \rightarrow L^{2}$ is invertible for all $\delta>0$. By considering sequences

$$
w_{j}=\left(\mathcal{L}_{\varepsilon_{j}}+\delta\right) v_{j}, \quad\left\|v_{j}\right\|_{H^{1}}=1, \quad \varepsilon_{j} \rightarrow 0
$$

that converge weakly to a pair

$$
w_{0}=\left(\mathcal{L}_{0}+\delta\right) v_{0},
$$

\footnotetext{
${ }^{1}$ The power of the results in [1] is that they also apply to variants of (1.9) with infinite-range interactions. We describe their ideas here in a finite-range setting for notational clarity.
} 
the authors show that also $\mathcal{L}_{\varepsilon}+\delta: H^{1} \rightarrow L^{2}$ is invertible. To this end one needs to establish a lower bound for $\left\|w_{0}\right\|_{L^{2}}$, which can be achieved by exploiting inequalities of the form

$$
\langle v(\cdot+\varepsilon)+v(\cdot-\varepsilon)-2 v(\cdot), v(\cdot)\rangle_{L^{2}} \leq 0, \quad\left\langle v^{\prime}, v\right\rangle_{L^{2}}=0,
$$

and using the bistable structure of the nonlinearity $g$.

In [44] we showed that these ideas can be generalized to infinite-range versions of the FitzHugh-Nagumo LDE (1.7). The key issue there, which we must also face in this paper, is that problematic cross terms arise that must be kept under control when taking inner products. We are aided in this respect by the fact that the off-diagonal terms in the linearization of (1.1) are constant multiples of each other.

A second key complication that we encounter here is that the scale separation in the diffusion terms prevents us from using the direct multicomponent analogue of the inequality (1.23). We must carefully include $\varepsilon$-dependent weights into our inner products to compensate for these imbalances. This complicates the fixed-point argument used to control the nonlinear terms during the construction of the traveling waves. In fact, it forces us to take an additional spatial derivative of the traveling wave equations.

This latter situation was also encountered in [31], where the spectral convergence method was used to construct traveling wave solutions to adaptive-grid discretizations of the Nagumo PDE (1.18). Further applications of this technique can be found in $[32,42]$, where full spatial-temporal discretizations of the Nagumo PDE (1.18) and the FitzHugh-Nagumo PDE (1.4) are considered.

Overview. After stating our main results in section 2 we apply the spectral convergence method discussed above to the system of traveling wave equations (1.12) in sections 3 and 4 . This allows us to follow the spirit of [1, Thm. 1] to establish the existence of traveling waves in section 5. In particular, we use a fixed point argument that mimics the proof of the standard implicit function theorem.

We follow the approach developed in [44] to analyze the spectral stability of these traveling waves in section 6 . In particular, we recycle the spectral convergence argument to analyze the linear operators $\overline{\mathcal{L}}_{\varepsilon}$ that arise after linearizing (1.12) around the newfound waves, instead of around the singular pulse $\bar{U}_{0}$ defined in (1.16). The key complication here is that for fixed small values of $\varepsilon>0$ we need results on the invertibility of $\overline{\mathcal{L}}_{\varepsilon}+\lambda$ for all $\lambda$ in a half-strip. By contrast, the spectral convergence method gives a range of admissible values for $\varepsilon>0$ for each fixed $\lambda$. Switching between these two points of view is a delicate task, but fortunately the main ideas from [44] can be transferred to this setting.

The nonlinear stability of the traveling waves can be inferred from their spectral stability in a relatively straightforward fashion by appealing to the theory developed in [30] for discrete systems with finite range interactions. A more detailed description of this procedure in an infinite-range setting can be found in [43, sections 7-8].

2. Main results. Our main results concern the LDE

$$
\begin{aligned}
& \dot{u}_{j}(t)=d_{j} \mathcal{D}\left[u_{j+1}(t)+u_{j-1}(t)-2 u_{j}(t)\right]+f_{j}\left(u_{j}(t), w_{j}(t)\right), \\
& \dot{w}_{j}(t)=g_{j}\left(u_{j}(t), w_{j}(t)\right)
\end{aligned}
$$

posed on the one-dimensional lattice $j \in \mathbb{Z}$, where we take $u_{j} \in \mathbb{R}^{n}$ and $w_{j} \in \mathbb{R}^{k}$ for some pair of integers $n \geq 1$ and $k \geq 0$. We assume that the system is 2-periodic in 
the sense that there exists a set of four nonlinearities

$$
f_{o}: \mathbb{R}^{n+k} \rightarrow \mathbb{R}^{n}, \quad f_{e}: \mathbb{R}^{n+k} \rightarrow \mathbb{R}^{n}, \quad g_{o}: \mathbb{R}^{n+k} \rightarrow \mathbb{R}^{k}, \quad g_{e}: \mathbb{R}^{n+k} \rightarrow \mathbb{R}^{k}
$$

for which we may write

$$
\left(d_{j}, f_{j}, g_{j}\right)=\left\{\begin{array}{r}
\left(\varepsilon^{-2}, f_{o}, g_{o}\right) \text { for odd } j \\
\left(1, f_{e}, g_{e}\right) \text { for even } j .
\end{array}\right.
$$

Introducing the shorthand notation

$$
F_{o}(u, w)=\left(f_{o}(u, w), g_{o}(u, w)\right), \quad F_{e}(u, w)=\left(f_{e}(u, w), g_{e}(u, w)\right),
$$

we impose the following structural condition on our system that concerns the roots of the nonlinearities $F_{o}$ and $F_{e}$. These roots correspond with temporal equilibria of (2.1) that have a spatially homogeneous $u$-component. On the other hand, the $w$-component of these equilibria is allowed to be 2-periodic.

Assumption (HN1). The matrix $\mathcal{D} \in \mathbb{R}^{n \times n}$ is a diagonal matrix with strictly positive diagonal entries. In addition, the nonlinearities $F_{o}$ and $F_{e}$ are $C^{3}$-smooth and there exist four vectors

$$
U_{e}^{ \pm}=\left(u_{e}^{ \pm}, w_{e}^{ \pm}\right) \in \mathbb{R}^{n+k}, \quad U_{o}^{ \pm}=\left(u_{o}^{ \pm}, w_{o}^{ \pm}\right) \in \mathbb{R}^{n+k},
$$

for which we have the identities $u_{o}^{-}=u_{e}^{-}$and $u_{o}^{+}=u_{e}^{+}$, together with

$$
F_{o}\left(U_{o}^{ \pm}\right)=F_{e}\left(U_{e}^{ \pm}\right)=0 .
$$

We emphasize that any subset of the four vectors $U_{o}^{ \pm}$and $U_{e}^{ \pm}$is allowed to be identical. In order to address the temporal stability of these equilibria, we introduce two separate auxiliary conditions on triplets

$$
\left(G, U^{-}, U^{+}\right) \in C^{1}\left(\mathbb{R}^{n+k} ; \mathbb{R}^{n+k}\right) \times \mathbb{R}^{n+k} \times \mathbb{R}^{n+k},
$$

which are both stronger ${ }^{2}$ than the requirement that all the eigenvalues of $D G\left(U^{ \pm}\right)$ have strictly negative real parts. As can be seen, the block structure of this matrix plays an important role in $(\mathrm{h} \beta)$, which is why we have chosen to state our results for arbitrary values of $n \geq 1$ and $k \geq 0$.

Assumption $(\mathrm{H} \alpha)$. The matrices $-D G\left(U^{-}\right)$and $-D G\left(U^{+}\right)$are positive definite.

Assumption $(\mathrm{H} \beta)$. For any $U \in \mathbb{R}^{n+k}$, write $D G(U)$ in the block form

$$
D G(U)=\left(\begin{array}{ll}
G_{1,1}(U) & G_{1,2}(U) \\
G_{2,1}(U) & G_{2,2}(U)
\end{array}\right)
$$

with $G_{1,1}(U) \in \mathbb{R}^{n \times n}$. Then the matrices $-G_{1,1}\left(U^{-}\right),-G_{1,1}\left(U^{+}\right),-G_{2,2}\left(U^{-}\right)$, and $-G_{2,2}\left(U^{+}\right)$are positive definite. In addition, there exists a constant $\Gamma>0$ so that $G_{1,2}(U)=-\Gamma G_{2,1}(U)^{T}$ holds for all $U \in \mathbb{R}^{n \times k}$.

As an illustration, we pick $0<a<1$ and write

$$
G_{\text {ngm }}(u)=u(1-u)(u-a)
$$

\footnotetext{
${ }^{2}$ See the proof of Lemma 4.6 for details.
} 
for the nonlinearity associated with the Nagumo equation, together with

$$
G_{\mathrm{fhn} ; \rho, \gamma}(u, w)=\left(\begin{array}{l}
u(1-u)(u-a)-w \\
\rho[u-\gamma w]
\end{array}\right)
$$

for its counterpart corresponding to the FitzHugh-Nagumo system. It can be easily verified that the triplet $\left(G_{\mathrm{ngm}}, 0,1\right)$ satisfies $\left((\mathrm{h} \alpha)\right.$, while the triplet $\left(G_{\mathrm{fhn} ; \rho, \gamma}, 0,0\right)$ satisfies $(\mathrm{h} \beta)$ for $\rho>0$ and $\gamma>0$ with $\Gamma=\rho^{-1}$. When $a>0$ is sufficiently small, the Jacobian $D G_{\text {fhn; } \rho, \gamma}(0,0)$ has a pair of complex eigenvalues with negative real part. In this case $(\mathrm{h} \alpha)$ may fail to hold.

The following assumption states that the even and odd subsystems must both satisfy one of the two auxiliary conditions above. We emphasize, however, that this does not necessarily need to be the same condition for both systems.

Assumption (HN2). The triplet $\left(F_{o}, U_{o}^{-}, U_{o}^{+}\right)$satisfies either $(h \alpha)$ or $(h \beta)$. The same holds for the triplet $\left(F_{e}, U_{e}^{-}, U_{e}^{+}\right)$.

We intend to find functions

$$
\left(u_{\varepsilon}, w_{\varepsilon}\right): \mathbb{R} \rightarrow \ell^{\infty}\left(\mathbb{Z} ; \mathbb{R}^{n}\right) \times \ell^{\infty}\left(\mathbb{Z} ; \mathbb{R}^{k}\right)
$$

that take the form

$$
\left(u_{\varepsilon}, w_{\varepsilon}\right)_{j}(t)= \begin{cases}\left(\bar{u}_{o ; \varepsilon}, \bar{w}_{o ; \varepsilon}\right)\left(j+c_{\varepsilon} t\right) & \text { for odd } j \\ \left(\bar{u}_{e ; \varepsilon}, \bar{w}_{e ; \varepsilon}\right)\left(j+c_{\varepsilon} t\right) & \text { for even } j\end{cases}
$$

and satisfy (2.1) for all $t \in \mathbb{R}$. The waveprofiles are required to be $C^{1}$-smooth and satisfy the limits

$$
\lim _{\xi \rightarrow \pm \infty}\left(\bar{u}_{o}(\xi), \bar{w}_{o}(\xi)\right)=\left(u_{o}^{ \pm}, w_{o}^{ \pm}\right), \quad \quad \lim _{\xi \rightarrow \pm \infty}\left(\bar{u}_{e}(\xi), \bar{w}_{e}(\xi)\right)=\left(u_{e}^{ \pm}, w_{e}^{ \pm}\right) .
$$

Substituting the traveling wave Ansatz (2.12) into the LDE (2.1) yields the coupled system

$$
\begin{aligned}
c_{\varepsilon} \bar{u}_{o ; \varepsilon}^{\prime}(\xi) & =\frac{1}{\varepsilon^{2}} \mathcal{D} \Delta_{\text {mix }}\left[\bar{u}_{o ; \varepsilon}, \bar{u}_{e ; \varepsilon}\right](\xi)+f_{o}\left(\bar{u}_{o ; \varepsilon}(\xi), \bar{w}_{o ; \varepsilon}(\xi)\right), \\
c_{\varepsilon} \bar{w}_{o ; \varepsilon}^{\prime}(\xi) & =g_{o}\left(\bar{u}_{o ; \varepsilon}(\xi), \bar{w}_{o ; \varepsilon}(\xi)\right), \\
c_{\varepsilon} \bar{u}_{e ; \varepsilon}^{\prime}(\xi) & =\mathcal{D} \Delta_{\text {mix }}\left[\bar{u}_{e ; \varepsilon}, \bar{u}_{o ; \varepsilon}\right](\xi)+f_{e}\left(\bar{u}_{e ; \varepsilon}(\xi), \bar{w}_{e ; \varepsilon}(\xi)\right), \\
c_{\varepsilon} \bar{w}_{e ; \varepsilon}^{\prime}(\xi) & =g_{e}\left(\bar{u}_{e ; \varepsilon}(\xi), \bar{w}_{e ; \varepsilon}(\xi)\right)
\end{aligned}
$$

in which we have introduced the shorthand

$$
\Delta_{\text {mix }}[\phi, \psi](\xi)=\psi(\xi+1)+\psi(\xi-1)-2 \phi(\xi) .
$$

Multiplying the first line of (2.14) by $\varepsilon^{2}$ and taking the formal limit $\varepsilon \downarrow 0$, we obtain the identity

$$
0=\mathcal{D} \Delta_{\text {mix }}\left[\bar{u}_{o ; 0}, \bar{u}_{e ; 0}\right](\xi)
$$

which can be explicitly solved to yield

$$
\bar{u}_{o ; 0}(\xi)=\frac{1}{2} \bar{u}_{e ; 0}(\xi+1)+\frac{1}{2} \bar{u}_{e ; 0}(\xi-1) .
$$

Copyright (C) by SIAM. Unauthorized reproduction of this article is prohibited. 
In the $\varepsilon \downarrow 0$ limit, the even subsystem of (2.14) hence decouples and becomes

$$
\begin{aligned}
c_{0} \bar{u}_{e ; 0}^{\prime}(\xi) & =\frac{1}{2} \mathcal{D}\left[\bar{u}_{e ; 0}(\xi+2)+\bar{u}_{e ; 0}(\xi-2)-2 \bar{u}_{e ; 0}(\xi)\right]+f_{e}\left(\bar{u}_{e ; 0}(\xi), \bar{w}_{e ; 0}(\xi)\right), \\
c_{0} \bar{w}_{e ; 0}^{\prime}(\xi) & =g_{e}\left(\bar{u}_{e ; 0}(\xi), \bar{w}_{e ; 0}(\xi)\right) .
\end{aligned}
$$

We require this limiting even system to have a traveling wave solution that connects $U_{e}^{-}$to $U_{e}^{+}$.

Assumption (HW1). There exists $c_{0} \neq 0$ for which the system (2.18) has a $C^{1}$-smooth solution $\bar{U}_{e ; 0}=\left(\bar{u}_{e ; 0}, \bar{w}_{e ; 0}\right)$ that satisfies the limits

$$
\lim _{\xi \rightarrow \pm \infty}\left(\bar{u}_{e ; 0}(\xi), \bar{w}_{e ; 0}(\xi)\right)=\left(u_{e}^{ \pm}, w_{e}^{ \pm}\right)
$$

Finally, taking $\varepsilon \downarrow 0$ in the second line of (2.14) and applying (2.17), we obtain the identity

$$
c_{0} \bar{w}_{o ; 0}^{\prime}(\xi)=g_{o}\left(\frac{1}{2} \bar{u}_{e ; 0}(\xi+1)+\frac{1}{2} \bar{u}_{e ; 0}(\xi-1), \bar{w}_{o ; 0}(\xi)\right)
$$

in which $\bar{w}_{o ; 0}$ is the only remaining unknown. We impose the following compatibility condition on this system.

Assumption (HW2). Equation (2.20) has a $C^{1}$-smooth solution $\bar{w}_{o ; 0}$ that satisfies the limits

$$
\lim _{\xi \rightarrow \pm \infty} \bar{w}_{o ; 0}(\xi)=w_{o}^{ \pm}
$$

Upon writing

$$
\bar{U}_{0}=\left(\bar{U}_{o ; 0}, \bar{U}_{e ; 0}\right)=\left(\bar{u}_{o ; 0}, \bar{w}_{o ; 0}, \bar{u}_{e ; 0}, \bar{w}_{e ; 0}\right),
$$

we intend to seek a branch of solutions to (2.14) that bifurcates off the singular traveling wave $\left(\bar{U}_{0}, c_{0}\right)$. In view of the limits

$$
\lim _{\xi \rightarrow \pm \infty}\left(\bar{U}_{o ; 0}, \bar{U}_{e ; 0}\right)(\xi)=\left(U_{o}^{ \pm}, U_{e}^{ \pm}\right)
$$

we introduce the spaces

$$
\mathbf{H}_{e}^{1}=\mathbf{H}_{o}^{1}=H^{1}\left(\mathbb{R} ; \mathbb{R}^{n}\right) \times H^{1}\left(\mathbb{R} ; \mathbb{R}^{k}\right), \quad \mathbf{L}_{e}^{2}=\mathbf{L}_{o}^{2}=L^{2}\left(\mathbb{R} ; \mathbb{R}^{n}\right) \times L^{2}\left(\mathbb{R} ; \mathbb{R}^{k}\right)
$$

to analyze the perturbations from $\bar{U}_{0}$.

Linearizing (2.18) around the solution $\bar{U}_{e ; 0}$, we obtain the linear operator $\bar{L}_{e}$ : $\mathbf{H}_{e}^{1} \rightarrow \mathbf{L}_{e}^{2}$ that acts as

$$
\bar{L}_{e}=c_{0} \frac{d}{d \xi}-D F_{e}\left(\bar{U}_{e ; 0}\right)-\frac{1}{2}\left(\begin{array}{ll}
\mathcal{D}\left(S_{2}-2\right) & 0 \\
0 & 0
\end{array}\right),
$$

in which we have introduced the notation

$$
\left[S_{2} \phi\right](\xi)=\phi(\xi+2)+\phi(\xi-2) .
$$

Our perturbation argument to construct solutions of (2.14) requires $\bar{L}_{e}$ to have an isolated simple eigenvalue at the origin.

Copyright $@$ ( ) by SIAM. Unauthorized reproduction of this article is prohibited. 
Assumption (HS1). There exists $\delta_{e}>0$ so that the operator $\bar{L}_{e}+\delta$ is a Fredholm operator with index 0 for each $0 \leq \delta<\delta_{e}$. It has a simple eigenvalue in $\delta=0$, i.e., we have $\operatorname{Ker}\left(\bar{L}_{e}\right)=\operatorname{span}\left(\bar{U}_{e ; 0}^{\prime}\right)$ and $\bar{U}_{e ; 0}^{\prime} \notin \operatorname{Range}\left(\bar{L}_{e}\right)$.

We are now ready to formulate our first main result, which states that (2.14) admits a branch of solutions for small $\varepsilon>0$ that converges to the singular wave $\left(\bar{U}_{0}, c_{0}\right)$ as $\varepsilon \downarrow 0$. Notice that the $\varepsilon$-scalings on the norms of $\Phi_{\varepsilon}^{\prime}$ and $\Phi_{\varepsilon}^{\prime \prime}$ are considerably better than those suggested by a direct inspection of (2.14).

Theorem 2.1 (see section 5). Assume that (HN1), (HN2), (HW1), (HW2), and (HS1) are satisfied. There exists a constant $\varepsilon_{*}>0$ so that for each $0<\varepsilon<\varepsilon_{*}$, there exist $c_{\varepsilon} \in \mathbb{R}$ and $\Phi_{\varepsilon}=\left(\Phi_{o ; \varepsilon}, \Phi_{e ; \varepsilon}\right) \in \mathbf{H}_{o}^{1} \times \mathbf{H}_{e}^{1}$ for which the function

$$
\bar{U}_{\varepsilon}=\bar{U}_{0}+\Phi_{\varepsilon}
$$

is a solution of the traveling wave system (2.14) with wave speed $c=c_{\varepsilon}$. In addition, we have the limit

$$
\lim _{\varepsilon \downarrow 0}\left[\left\|\varepsilon \Phi_{o ; \varepsilon}^{\prime \prime}\right\|_{\mathbf{L}_{o}^{2}}+\left\|\Phi_{e ; \varepsilon}^{\prime \prime}\right\|_{\mathbf{L}_{e}^{2}}+\left\|\Phi_{\varepsilon}^{\prime}\right\|_{\mathbf{L}_{o}^{2} \times \mathbf{L}_{e}^{2}}+\left\|\Phi_{\varepsilon}\right\|_{\mathbf{L}_{o}^{2} \times \mathbf{L}_{e}^{2}}+\left|c_{\varepsilon}-c_{0}\right|\right]=0
$$

and the function $\bar{U}_{\varepsilon}$ is locally unique up to translation.

In order to show that our newfound traveling wave solution is stable under the flow of the LDE (2.1), we need to impose the following extra assumption on the operator $\bar{L}_{e}$. To understand the restriction on $\lambda$, we recall that the spectrum of $\bar{L}_{e}$ admits the periodicity $\lambda \mapsto \lambda+2 \pi i c_{0}$.

Assumption (HS2). There exists a constant $\lambda_{e}>0$ so that the operator $\bar{L}_{e}+\lambda$ : $\mathbf{H}_{e}^{1} \rightarrow \mathbf{L}_{e}^{2}$ is invertible for all $\lambda \in \mathbb{C} \backslash 2 \pi i c_{0} \mathbb{Z}$ that have $\operatorname{Re} \lambda \geq-\lambda_{e}$.

Together with (HS1) this condition states that the wave $\left(\bar{U}_{e ; 0}, c_{0}\right)$ for the limiting even system (2.18) is spectrally stable. Our second main theorem shows that this can be generalized to a nonlinear stability result for the wave solutions (2.12) of the full system (2.1).

TheOREm 2.2 (see section 6). Assume that (HN1), (HN2), (HW1), (HW2), (HS1), and (HS2) are satisfied and pick a sufficiently small $\varepsilon>0$. Then there exist constants $\delta>0, C>0$, and $\beta>0$ so that for all $1 \leq p \leq \infty$ and all initial conditions

$$
\left(u^{0}, w^{0}\right) \in \ell^{p}\left(\mathbb{Z} ; \mathbb{R}^{n}\right) \times \ell^{p}\left(\mathbb{Z} ; \mathbb{R}^{k}\right)
$$

that admit the bound

$$
E_{0}:=\left\|u^{0}-u_{\varepsilon}(0)\right\|_{\ell^{p}\left(\mathbb{Z} ; \mathbb{R}^{n}\right)}+\left\|w^{0}-w_{\varepsilon}(0)\right\|_{\ell^{p}\left(\mathbb{Z} ; \mathbb{R}^{k}\right)}<\delta,
$$

there exists an asymptotic phase shift $\tilde{\theta} \in \mathbb{R}$ such that the solution $(u, w)$ of (2.1) with the initial condition $(u, w)(0)=\left(u^{0}, w^{0}\right)$ satisfies the estimate

$$
\left\|u(t)-u_{\varepsilon}(t+\tilde{\theta})\right\|_{\ell^{p}\left(\mathbb{Z} ; \mathbb{R}^{n}\right)}+\left\|w(t)-w_{\varepsilon}(t+\tilde{\theta})\right\|_{\ell^{p}\left(\mathbb{Z} ; \mathbb{R}^{k}\right)} \leq C e^{-\beta t} E_{0}
$$

for all $t>0$.

Our final result shows that our framework is broad enough to cover the twoperiodic FitzHugh-Nagumo system (1.1). We remark that the condition on $\gamma_{e}$ ensures that $(0,0)$ is the only spatially homogeneous equilibrium for the limiting even subsystem (1.14). This allows us to apply the spatially homogeneous results obtained in $[29,30]$. 
Corollary 2.3. Consider the LDE (1.1) and suppose that $\gamma_{o}>0$ and $\rho_{o}>0$ both hold. Suppose furthermore that $a_{e}$ is sufficiently far away from $\frac{1}{2}$, that $0<\gamma_{e}<$ $4\left(1-a_{e}\right)^{-2}$, and that $\rho_{e}>0$ is sufficiently small. Then for each sufficiently small $\varepsilon>0$, there exists a nonlinearly stable traveling pulse solution of the form (2.12) that satisfies the limits

$$
\lim _{\xi \rightarrow \pm \infty}\left(\bar{u}_{o}(\xi), \bar{w}_{o}(\xi)\right)=(0,0), \quad \quad \lim _{\xi \rightarrow \pm \infty}\left(\bar{u}_{e}(\xi), \bar{w}_{e}(\xi)\right)=(0,0) .
$$

Proof. Assumption (HN1) can be verified directly, while (HN2) follows from the discussion above concerning the nonlinearity $G_{\mathrm{fhn} ; \rho, \gamma}$ defined in (2.10). Assumption (HW1) follows from the existence theory developed in [29], while (HS1) and (HS2) follow from the spectral analysis in [30]. The remaining condition (HW2) can be verified by noting that the nonlinearity $g_{o}$ is in fact linear and invertible with respect to $\bar{w}_{o ; 0}$ on account of Lemma 3.5 below.

3. The limiting system. In this section we analyze the linear operator that is associated to the limiting system that arises by combining (2.18) and (2.20). In order to rewrite this system in a compact fashion, we introduce the notation

$$
\left[S_{i} \phi\right](\xi)=\phi(\xi+i)+\phi(\xi-i)
$$

together with the $(n+k) \times(n+k)$-matrix $J_{\mathcal{D}}$ that has the block structure

$$
J_{\mathcal{D}}=\left(\begin{array}{ll}
\mathcal{D} & 0 \\
0 & 0
\end{array}\right) .
$$

This allows us to recast (2.25) in the shortened form

$$
\bar{L}_{e}=c_{0} \frac{d}{d \xi}-\frac{1}{2} J_{\mathcal{D}}\left(S_{2}-2\right)-D F_{e}\left(\bar{U}_{e ; 0}\right) .
$$

One can associate a formal adjoint $\bar{L}_{e}^{\text {adj }}: \mathbf{H}_{e}^{1} \rightarrow \mathbf{L}_{e}^{2}$ to this operator by writing

$$
\bar{L}_{e}^{\mathrm{adj}}=-c_{0} \frac{d}{d \xi}-\frac{1}{2} J_{\mathcal{D}}\left(S_{2}-2\right)-D F_{e}\left(\bar{U}_{e ; 0}\right)^{T} .
$$

Assumption (HS1) together with the Fredholm theory developed in [39] implies that

$$
\operatorname{ind}\left(\bar{L}_{e}\right)=-\operatorname{ind}\left(\bar{L}_{e}^{\mathrm{adj}}\right)
$$

holds for the Fredholm indices of these operators, which are defined as

$$
\operatorname{ind}(L)=\operatorname{dim}(\operatorname{ker}(L))-\operatorname{codim}(\operatorname{Range}(L)) .
$$

In particular, (HS1) implies that there exists a function

$$
\bar{\Phi}_{e ; 0}^{\mathrm{adj}} \in \operatorname{Ker}\left(\bar{L}_{e}^{\mathrm{adj}}\right) \subset \mathbf{H}_{e}^{1}
$$

that can be normalized to have

$$
\left\langle\bar{U}_{e ; 0}^{\prime}, \bar{\Phi}_{e ; 0}^{\mathrm{adj}}\right\rangle_{\mathbf{L}_{e}^{2}}=1 .
$$

We also introduce the operator $\bar{L}_{o}: H^{1}\left(\mathbb{R} ; \mathbb{R}^{k}\right) \rightarrow L^{2}\left(\mathbb{R} ; \mathbb{R}^{k}\right)$ associated to the linearization of (2.20) around $\bar{U}_{o ; 0}$, which acts as

$$
\bar{L}_{o}=c_{0} \frac{d}{d \xi}-D_{2} g_{o}\left(\bar{U}_{o ; 0}\right) .
$$

Copyright $@$ by SIAM. Unauthorized reproduction of this article is prohibited. 
In order to couple this operator with $\bar{L}_{e}$, we introduce the spaces

$$
\mathbf{H}_{\diamond}^{1}=H^{1}\left(\mathbb{R} ; \mathbb{R}^{k}\right) \times \mathbf{H}_{e}^{1}, \quad \mathbf{L}_{\diamond}^{2}=L^{2}\left(\mathbb{R} ; \mathbb{R}^{k}\right) \times \mathbf{L}_{e}^{2},
$$

together with the operator

$$
\mathcal{L}_{\diamond ; \delta}: \mathbf{H}_{\diamond}^{1} \rightarrow \mathbf{L}_{\diamond}^{2}
$$

that acts as

$$
\mathcal{L}_{\diamond ; \delta}=\left(\begin{array}{cc}
\bar{L}_{o}+\delta & 0 \\
0 & \bar{L}_{e}+\delta
\end{array}\right) .
$$

Our first main result shows that $\mathcal{L}_{\diamond ; \delta}$ inherits several properties of $\bar{L}_{e}+\delta$.

Proposition 3.1. Assume that (HN1), (HN2), (HW1), (HW2), and (HS1) are satisfied. Then there exist constants $\delta_{\diamond}>0$ and $C_{\diamond}>0$ so that the following holds true:

(i) For every $0<\delta<\delta_{\diamond}$, the operator $\mathcal{L}_{\diamond, \delta}$ is invertible as a map from $\mathbf{H}_{\diamond}^{1}$ to $\mathbf{L}_{\diamond}^{2}$.

(ii) For any $\Theta_{\diamond} \in \mathbf{L}_{\diamond}^{2}$ and $0<\delta<\delta_{\diamond}$ the function $\Phi_{\diamond}=\mathcal{L}_{\diamond, \delta}^{-1} \Theta_{\diamond} \in \mathbf{H}_{\diamond}^{1}$ satisfies the bound

$$
\left\|\Phi_{\diamond}\right\|_{\mathbf{H}_{\diamond}^{1}} \leq C_{\diamond}\left[\left\|\Theta_{\diamond}\right\|_{\mathbf{L}_{\diamond}^{2}}+\frac{1}{\delta}\left|\left\langle\Theta_{\diamond},\left(0, \bar{\Phi}_{e ; 0}^{\mathrm{adj}}\right)\right\rangle_{\mathbf{L}_{\diamond}^{2}}\right|\right] .
$$

If (HS2) also holds, then we can consider compact sets $\lambda \in M \subset \mathbb{C}$ that avoid the spectrum of $\bar{L}_{e}$. To formalize this, we impose the following assumption on $M$ and state our second main result.

Assumption $\left(\mathrm{HM}_{\lambda_{0}}\right)$. The set $M \subset \mathbb{C}$ is compact with $2 \pi i c_{0} \mathbb{Z} \cap M=\emptyset$. In addition, we have $\operatorname{Re} \lambda \geq-\lambda_{0}$ for all $\lambda \in M$.

Proposition 3.2. Assume that (HN1), (HN2), (HW1), (HW2), (HS1), and (HS2) are all satisfied and pick a sufficiently small constant $\lambda_{\diamond}>0$. Then for any set $M \subset \mathbb{C}$ that satisfies $\left(h M_{\lambda_{0}}\right)$ for $\lambda_{0}=\lambda_{\diamond}$ there exists a constant $C_{\diamond ; M}>0$ so that the following holds true:

(i) For every $\lambda \in M$, the operator $\mathcal{L}_{\diamond, \lambda}$ is invertible as a map from $\mathbf{H}_{\diamond}^{1}$ to $\mathbf{L}_{\diamond}^{2}$.

(ii) For any $\Theta_{\diamond} \in \mathbf{L}_{\diamond}^{2}$ and $\lambda \in M$, the function $\Phi_{\diamond}=\mathcal{L}_{\diamond, \lambda}^{-1} \Theta_{\diamond} \in \mathbf{H}_{\diamond}^{1}$ satisfies the bound

$$
\left\|\Phi_{\diamond}\right\|_{\mathbf{H}_{\diamond}^{1}} \leq C_{\diamond ; M}\left\|\Theta_{\diamond}\right\|_{\mathbf{L}_{\diamond}^{2}}
$$

3.1. Properties of $\bar{L}_{o}$. The assumptions (HS1) and (HS2) already contain the information on $\bar{L}_{e}$ that we require to establish Propositions 3.1 and 3.2. Our task here is, therefore, to understand the operator $\bar{L}_{o}$. As a preparation, we show that the top-left and bottom-right corners of the limiting Jacobians $D F_{o}\left(U_{o}^{ \pm}\right)$are both negative definite, which will help us to establish useful Fredholm properties.

Lemma 3.3. Assume that (HN1) and (HN2) are both satisfied. Then the matrices $D_{1} f_{\#}\left(U_{\#}^{ \pm}\right)$and $D_{2} g_{\#}\left(U_{\#}^{ \pm}\right)$are all negative definite for each $\# \in\{o, e\}$.

Proof. Note first that $D_{1} f_{\#}$ and $D_{2} g_{\#}$ correspond with $G_{1,1}$, respectively, $G_{2 ; 2}$, in the block structure (2.8) for $D F_{\#}$. We hence see that the matrices $D_{1} f_{\#}\left(U_{\#}^{ \pm}\right)$and $D_{2} g_{\#}\left(U_{\#}^{ \pm}\right)$are negative definite, either directly by $(\mathrm{h} \beta)$ or by the fact that they are principal submatrices of $D F_{\#}\left(U_{\#}^{ \pm}\right)$, which are negative definite if $(\mathrm{h} \alpha)$ holds.

Copyright $\odot$ by SIAM. Unauthorized reproduction of this article is prohibited. 
Lemma 3.4. Assume that (HN1), (HN2), (HW1), and (HW2) are satisfied. Then there exists $\lambda_{o}>0$ so that the operator $\bar{L}_{o}+\lambda$ is Fredholm with index zero for each $\lambda \in \mathbb{C}$ with $\operatorname{Re} \lambda \geq-\lambda_{o}$.

Proof. For any $0 \leq \rho \leq 1$ and $\lambda \in \mathbb{C}$ we introduce the constant coefficient linear operator $L_{\rho, \lambda}: H^{1}\left(\mathbb{R} ; \mathbb{R}^{k}\right) \rightarrow L^{2}\left(\mathbb{R} ; \mathbb{R}^{k}\right)$ that acts as

$$
L_{\rho, \lambda}=c_{0} \frac{d}{d \xi}-\rho D_{2} g_{o}\left(U_{o}^{-}\right)-(1-\rho) D_{2} g_{o}\left(U_{o}^{+}\right)+\lambda
$$

and has the characteristic function

$$
\Delta_{L_{\rho, \lambda}}(z)=c_{0} z-\rho D_{2} g_{o}\left(U_{o}^{-}\right)-(1-\rho) D_{2} g_{o}\left(U_{o}^{+}\right)+\lambda .
$$

Upon introducing the matrix

$$
B_{\rho}=-\rho D_{2} g_{o}\left(U_{o}^{-}\right)-(1-\rho) D_{2} g_{o}\left(U_{o}^{+}\right)-\rho D_{2} g_{o}\left(U_{o}^{-}\right)^{T}-(1-\rho) D_{2} g_{o}\left(U_{o}^{+}\right)^{T},
$$

which is positive definite by Lemma 3.3 , we pick $\lambda_{o}>0$ in such a way that $B_{\rho}-2 \lambda_{o}$ remains positive definite for each $0 \leq \rho \leq 1$. It is easy to check that the identity

$$
\Delta_{L_{\rho, \lambda}}(i y)+\Delta_{L_{\rho, \lambda}}(i y)^{\dagger}=B_{\rho}+2 \operatorname{Re} \lambda
$$

holds for any $y \in \mathbb{R}$. Here we use the symbol $\dagger$ for the conjugate transpose matrix. In particular, if we assume that $\operatorname{Re} \lambda \geq-\lambda_{o}$ and that $\Delta_{L_{\rho, \lambda}}(i y) v_{o}=0$ for some nonzero $v_{o} \in \mathbb{C}^{k}, y \in \mathbb{R}$, and $0 \leq \rho \leq 1$, then we obtain the contradiction

$$
\begin{aligned}
0 & =\operatorname{Re}\left[v_{o}^{\dagger}\left[\Delta_{L_{\rho}}(i y)+\Delta_{L_{\rho}}(i y)^{\dagger}\right] v_{o}\right] \\
& =\operatorname{Re} v_{o}^{\dagger}\left[B_{\rho}+2 \operatorname{Re} \lambda\right] v_{o} \\
& >0 .
\end{aligned}
$$

Using [39, Thm. A] together with the spectral flow principle in [39, Thm. C], this implies that $\bar{L}_{o}+\lambda$ is a Fredholm operator with index zero.

Lemma 3.5. Assume that (HN1), (HN2), (HW1), and (HW2) are satisfied and pick a sufficiently small constant $\lambda_{o}>0$. Then for any $\lambda \in \mathbb{C}$ with $\operatorname{Re} \lambda \geq-\lambda_{o}$ the operator $\bar{L}_{o}+\lambda$ is invertible as a map from $H^{1}\left(\mathbb{R} ; \mathbb{R}^{k}\right)$ into $L^{2}\left(\mathbb{R} ; \mathbb{R}^{k}\right)$. In addition, for each compact set

$$
M \subset\left\{\lambda: \operatorname{Re} \lambda \geq-\lambda_{o}\right\} \subset \mathbb{C}
$$

there exists a constant $K_{M}>0$ so that the uniform bound

$$
\left\|\left[\bar{L}_{o}+\lambda\right]^{-1} \chi_{o}\right\|_{H^{1}\left(\mathbb{R} ; \mathbb{R}^{k}\right)} \leq K_{M}\left\|\chi_{o}\right\|_{L^{2}\left(\mathbb{R} ; \mathbb{R}^{k}\right)}
$$

holds for any $\chi_{o} \in L^{2}\left(\mathbb{R} ; \mathbb{R}^{k}\right)$ and any $\lambda \in M$.

Proof. Recall the constant $\lambda_{o}$ defined in Lemma 3.4 and pick any $\lambda \in \mathbb{C}$ with $\operatorname{Re} \lambda \geq-\lambda_{o}$. On account of Lemma 3.4 it suffices to show that $\bar{L}_{o}+\lambda$ is injective. Consider therefore any nontrivial $\psi \in \operatorname{Ker}\left(\bar{L}_{o}+\lambda\right)$, which necessarily satisfies the ordinary differential equation $(\mathrm{ODE})^{3}$

$$
\psi^{\prime}(\xi)=\frac{1}{c_{0}} D_{2} g_{o}\left(\bar{U}_{o ; 0}(\xi)\right) \psi(\xi)-\frac{\lambda}{c_{0}} \psi(\xi)
$$

posed on $\mathbb{C}^{k}$. Without loss of generality we may assume that $c_{0}>0$.

\footnotetext{
${ }^{3}$ The discussion at https://math.stackexchange.com/questions/2668795/bounded-solution-togeneral-nonautonomous-ode gave us the inspiration for this approach.
}

Copyright (c) by SIAM. Unauthorized reproduction of this article is prohibited. 
Since $\bar{U}_{o ; 0}(\xi) \rightarrow U_{o}^{ \pm}$as $\xi \rightarrow \pm \infty$, Lemma 3.3 allows us to pick a constant $m \gg 1$ in such a way that the matrix $-D_{2} g_{o}\left(\bar{U}_{o ; 0}(\xi)\right)-2 \lambda_{o}$ is positive definite for each $|\xi| \geq m$, possibly after decreasing the size of $\lambda_{o}>0$. Assuming that $\operatorname{Re} \lambda \geq-\lambda_{o}$ and picking any $\xi \leq-m$, we may hence compute

$$
\begin{aligned}
\frac{d}{d \xi}|\psi(\xi)|^{2} & =2 \operatorname{Re}\left\langle\psi^{\prime}(\xi), \psi(\xi)\right\rangle_{\mathbb{C}^{k}} \\
& =\frac{2}{c_{0}} \operatorname{Re}\left\langle D_{2} g_{o}\left(\bar{U}_{o ; 0}(\xi)\right) \psi(\xi), \psi(\xi)\right\rangle_{\mathbb{C}^{k}}-\frac{2 \operatorname{Re} \lambda}{c_{0}}\langle\psi(\xi), \psi(\xi)\rangle_{\mathbb{C}^{k}} \\
& \leq-\frac{2 \lambda_{o}}{c_{0}}|\psi(\xi)|^{2}
\end{aligned}
$$

which implies that

$$
\left(e^{\frac{2 \lambda_{o}}{c_{0}} \xi}|\psi(\xi)|^{2}\right)^{\prime} \leq 0
$$

Since $\psi$ cannot vanish anywhere as a nontrivial solution to a linear ODE, we have

$$
|\psi(\xi)|^{2} \geq e^{-\frac{2 \lambda_{o}}{c_{0}}(m+\xi)}|\psi(-m)|^{2}>0
$$

for $\xi \leq-m$, which means that $\psi(\xi)$ is unbounded. In particular, we see that $\psi \notin$ $H^{1}\left(\mathbb{R} ; \mathbb{R}^{k}\right)$, which leads to the desired contradiction. The uniform bound (3.21) follows easily from continuity considerations.

Proof of Proposition 3.1. Since the operator $\bar{L}_{e}$ defined in (2.25) has a simple eigenvalue in zero, we can follow the approach of [44, Lem. 3.1(5)] to pick two constants $\delta_{\diamond}>0$ and $C>0$ in such a way that $\bar{L}_{e}+\delta: \mathbf{H}_{e}^{1} \rightarrow \mathbf{L}_{e}^{2}$ is invertible with the bound

$$
\left\|\left[\bar{L}_{e}+\delta\right]^{-1}\left(\theta_{e}, \chi_{e}\right)\right\|_{\mathbf{H}_{e}^{1}} \leq C\left[\left\|\left(\theta_{e}, \chi_{e}\right)\right\|_{\mathbf{L}_{e}^{2}}+\frac{1}{\delta}\left|\left\langle\left(\theta_{e}, \chi_{e}\right), \bar{\Phi}_{e ; 0}^{\mathrm{adj}}\right\rangle_{\mathbf{L}_{e}^{2}}\right|\right]
$$

for any $0<\delta<\delta_{\diamond}$ and $\left(\theta_{e}, \chi_{e}\right) \in \mathbf{L}_{e}^{2}$. Combining this estimate with Lemma 3.5 directly yields the desired properties.

Proof of Proposition 3.2. These properties can be established in a fashion analogous to the proof of Proposition 3.1.

4. Transfer of Fredholm properties. Our goal in this section is to lift the bounds obtained in section 3 to the operators associated to the linearization of the full wave equation (2.14) around suitable functions. In particular, the arguments we develop here will be used in several different settings. In order to accommodate this, we introduce the following condition.

Assumption (hFAm). For each $\varepsilon>0$ there is a function $\tilde{U}_{\varepsilon}=\left(\tilde{U}_{o ; \varepsilon}, \tilde{U}_{e ; \varepsilon}\right) \in$ $\mathbf{H}_{o}^{1} \times \mathbf{H}_{e}^{1}$ and a constant $\tilde{c}_{\varepsilon} \neq 0$ such that $\tilde{U}_{\varepsilon}-\bar{U}_{0} \rightarrow 0$ in $\mathbf{H}_{o}^{1} \times \mathbf{H}_{e}^{1}$ and $\tilde{c}_{\varepsilon} \rightarrow c_{0}$ as $\varepsilon \downarrow 0$. In addition, there exists a constant $\tilde{K}_{\mathrm{fam}}>0$ so that

$$
\left|\tilde{c}_{\varepsilon}\right|+\left|\tilde{c}_{\varepsilon}^{-1}\right|+\left\|\tilde{U}_{\varepsilon}\right\|_{\infty} \leq \tilde{K}_{\mathrm{fam}}
$$

holds for all $\varepsilon>0$.

In section 5 we will pick $\tilde{U}_{\varepsilon}=\bar{U}_{0}$ and $\tilde{c}_{\varepsilon}=c_{0}$ in (hFam) for all $\varepsilon>0$. On the other hand, in section 6 we will use the traveling wave solutions described in 
Theorem 2.1 to write $\tilde{U}_{\varepsilon}=\bar{U}_{\varepsilon}$ and $\tilde{c}_{\varepsilon}=c_{\varepsilon}$. We remark that (4.1) implies that there exists a constant $\tilde{K}_{F}>0$ for which the bound

$$
\left\|D F_{o}\left(\tilde{U}_{o ; \varepsilon}\right)\right\|_{\infty}+\left\|D^{2} F_{o}\left(\tilde{U}_{o ; \varepsilon}\right)\right\|_{\infty}+\left\|D F_{e}\left(\tilde{U}_{e ; \varepsilon}\right)\right\|_{\infty}+\left\|D^{2} F_{e}\left(\tilde{U}_{e ; \varepsilon}\right)\right\|_{\infty} \leq \tilde{K}_{F}
$$

holds for all $\varepsilon>0$.

For notational convenience, we introduce the product spaces

$$
\mathbf{H}^{1}=\mathbf{H}_{o}^{1} \times \mathbf{H}_{e}^{1}, \quad \mathbf{L}^{2}=\mathbf{L}_{o}^{2} \times \mathbf{L}_{e}^{2} .
$$

Since we will need to consider complex-valued functions during our spectral analysis, we also introduce the spaces

$$
\begin{aligned}
\mathbf{L}_{\mathbb{C}}^{2} & =\left\{\Phi+i \Psi: \Phi, \Psi \in \mathbf{L}^{2}\right\}, \\
\mathbf{H}_{\mathbb{C}}^{1} & =\left\{\Phi+i \Psi: \Phi, \Psi \in \mathbf{H}^{1}\right\}
\end{aligned}
$$

and remark that any $L \in \mathcal{L}\left(\mathbf{H}^{1} ; \mathbf{L}^{2}\right)$ can be interpreted as an operator in $\mathcal{L}\left(\mathbf{H}_{\mathbb{C}}^{1} ; \mathbf{L}_{\mathbb{C}}^{2}\right)$ by writing

$$
L(\Phi+i \Psi)=L \Phi+i L \Psi
$$

It is well-known that taking the complexification of an operator preserves injectivity, invertibility, and other Fredholm properties.

Recall the family $\left(\tilde{U}_{\varepsilon}, \tilde{c}_{\varepsilon}\right)$ introduced in (hFam). For any $\varepsilon>0$ and $\lambda \in \mathbb{C}$ we introduce the linear operator

$$
\tilde{\mathcal{L}}_{\varepsilon, \lambda}: \mathbf{H}_{\mathbf{C}}^{1} \rightarrow \mathbf{L}_{\mathbf{C}}^{2}
$$

that acts as

$$
\tilde{\mathcal{L}}_{\varepsilon, \lambda}=\left(\begin{array}{ll}
\tilde{c}_{\varepsilon} \frac{d}{d \xi}+\frac{2}{\varepsilon^{2}} J_{\mathcal{D}}-D F_{o}\left(\tilde{U}_{o ; \varepsilon}\right)+\lambda & -\frac{1}{\varepsilon^{2}} J_{\mathcal{D}} S_{1} \\
-J_{\mathcal{D}} S_{1} & \tilde{c}_{\varepsilon} \frac{d}{d \xi}+2 J_{\mathcal{D}}-D F_{e}\left(\tilde{U}_{e ; \varepsilon}\right)+\lambda
\end{array}\right) .
$$

In order to simplify our notation, we introduce the diagonal matrices

$$
\begin{aligned}
\mathcal{M}_{\varepsilon}^{1} & =\operatorname{diag}(\varepsilon, 1,1,1), \\
\mathcal{M}_{\varepsilon}^{2} & =\operatorname{diag}(1, \varepsilon, 1,1), \\
\mathcal{M}_{\varepsilon}^{1,2} & =\operatorname{diag}(\varepsilon, \varepsilon, 1,1) .
\end{aligned}
$$

In addition, we recall the sum $S_{1}$ defined in (3.1) and introduce the operator

$$
J_{\text {mix }}=\left(\begin{array}{cc}
-2 J_{\mathcal{D}} & J_{\mathcal{D}} S_{1} \\
J_{\mathcal{D}} S_{1} & -2 J_{\mathcal{D}}
\end{array}\right)
$$

which allows us to restate (4.7) as

$$
\tilde{\mathcal{L}}_{\varepsilon, \lambda}=\tilde{c}_{\varepsilon} \frac{d}{d \xi}-\mathcal{M}_{1 / \varepsilon^{2}}^{1} J_{\text {mix }}-D F\left(\tilde{U}_{\varepsilon}\right)+\lambda .
$$

Our two main results generalize the bounds in Propositions 3.1 and 3.2 to the current setting. The scalings on the odd variables allow us to obtain certain key estimates that are required by the spectral convergence approach.

Copyright (c) by SIAM. Unauthorized reproduction of this article is prohibited. 
Proposition 4.1. Assume that (hFam), (HN1), (HN2), (HW1), (HW2), and (HS1) are satisfied. Then there exist positive constants $C_{0}>0$ and $\delta_{0}>0$ together with a strictly positive function $\varepsilon_{0}:\left(0, \delta_{0}\right) \rightarrow \mathbb{R}_{>0}$, so that for each $0<\delta<\delta_{0}$ and $0<\varepsilon<\varepsilon_{0}(\delta)$ the operator $\tilde{\mathcal{L}}_{\varepsilon, \delta}$ is invertible and satisfies the bound

$$
\left\|\mathcal{M}_{\varepsilon}^{1,2} \Phi\right\|_{\mathbf{H}^{1}} \leq C_{0}\left[\left\|\mathcal{M}_{\varepsilon}^{1,2} \Theta\right\|_{\mathbf{L}^{2}}+\frac{1}{\delta}\left|\left\langle\Theta,\left(0, \bar{\Phi}_{e ; 0}^{\mathrm{adj}}\right)\right\rangle_{\mathbf{L}^{2}}\right|\right]
$$

for any $\Phi \in \mathbf{H}^{1}$ and $\Theta=\tilde{\mathcal{L}}_{\varepsilon, \delta} \Phi$.

Proposition 4.2. Assume that (hFam), (HN1), (HN2), (HW1), (HW2), (HS1), and (HS2) are all satisfied and pick a sufficiently small constant $\lambda_{0}>0$. Then for any set $M \subset \mathbb{C}$ that satisfies $\left(h M_{\lambda_{0}}\right)$, there exist positive constants $C_{M}>0$ and $\varepsilon_{M}>0$ so that for each $\lambda \in M$ and $0<\varepsilon<\varepsilon_{M}$ the operator $\tilde{\mathcal{L}}_{\varepsilon, \lambda}$ is invertible and satisfies the bound

$$
\left\|\mathcal{M}_{\varepsilon}^{1,2} \Phi\right\|_{\mathbf{H}_{\mathbb{C}}^{1}} \leq C_{M}\left\|\mathcal{M}_{\varepsilon}^{1,2} \Theta\right\|_{\mathbf{L}_{\mathbb{C}}^{2}}
$$

for any $\Phi \in \mathbf{H}_{\mathbb{C}}^{1}$ and $\Theta=\tilde{\mathcal{L}}_{\varepsilon, \lambda} \Phi$.

By using bootstrapping techniques it is possible to obtain variants of the estimate in Proposition 4.1. Indeed, it is possible to remove the scaling on the first component of $\Phi$ (but not on the first component of $\Phi^{\prime}$ ).

Corollary 4.3. Consider the setting of Proposition 4.1. Then for each $0<\delta<$ $\delta_{0}$ and $0<\varepsilon<\varepsilon_{0}(\delta)$, the operator $\tilde{\mathcal{L}}_{\varepsilon, \delta}$ satisfies the bound

$$
\left\|\mathcal{M}_{\varepsilon}^{1,2} \Phi^{\prime}\right\|_{\mathbf{L}^{2}}+\left\|\mathcal{M}_{\varepsilon}^{2} \Phi\right\|_{\mathbf{L}^{2}} \leq C_{0}\left[\left\|\mathcal{M}_{\varepsilon}^{1,2} \Theta\right\|_{\mathbf{L}^{2}}+\frac{1}{\delta}\left|\left\langle\Theta,\left(0, \bar{\Phi}_{e ; 0}^{\mathrm{adj}}\right)\right\rangle_{\mathbf{L}^{2}}\right|\right]
$$

for any $\Phi \in \mathbf{H}^{1}$ and $\Theta=\tilde{\mathcal{L}}_{\varepsilon, \delta} \Phi$, possibly after increasing $C_{0}>0$.

Proof. Write $\Phi=\left(\phi_{o}, \psi_{o}, \phi_{e}, \psi_{e}\right)$ and $\Theta=\left(\theta_{o}, \chi_{o}, \theta_{e}, \chi_{e}\right)$. Note that the first component of the equation $\Theta=\tilde{\mathcal{L}}_{\varepsilon, \delta} \Phi$ yields

$$
2 \mathcal{D} \phi_{o}=\mathcal{D} S_{1} \phi_{e}-\varepsilon^{2} \tilde{c}_{\varepsilon} \phi_{o}^{\prime}+\varepsilon^{2} D_{1} f_{o}\left(\tilde{U}_{o ; \varepsilon}\right) \phi_{o}+\varepsilon^{2} D_{2} f_{o}\left(\tilde{U}_{o ; \varepsilon}\right) \psi_{o}-\delta \varepsilon^{2} \phi_{o}+\varepsilon^{2} \theta_{o} .
$$

Recall the constants $\tilde{K}_{\text {fam }}$ and $\tilde{K}_{F}$ from (4.1) and (4.2), respectively, and write

$$
d_{\min }=\min _{1 \leq i \leq n} \mathcal{D}_{i, i}, \quad \quad d_{\max }=\max _{1 \leq i \leq n} \mathcal{D}_{i, i}
$$

We can now estimate

$$
\begin{aligned}
2 d_{\min }\left\|\phi_{o}\right\|_{L^{2}\left(\mathbb{R} ; \mathbb{R}^{n}\right) \leq} \leq & \left\|\mathcal{D} \phi_{o}\right\|_{L^{2}\left(\mathbb{R} ; \mathbb{R}^{n}\right)} \\
\leq \| & \left\|\mathcal{D} S_{1} \phi_{e}\right\|_{L^{2}\left(\mathbb{R} ; \mathbb{R}^{n}\right)}+\varepsilon \mid \tilde{c}_{\varepsilon}\left\|\varepsilon \phi_{o}^{\prime}\right\|_{L^{2}\left(\mathbb{R} ; \mathbb{R}^{n}\right)} \\
& \quad+\varepsilon\left\|D_{1} f_{o}\left(\bar{U}_{o ; \varepsilon}\right)\right\|_{\infty}\left\|\varepsilon \phi_{o}\right\|_{L^{2}\left(\mathbb{R} ; \mathbb{R}^{n}\right)} \\
& \quad+\varepsilon\left\|D_{2} f_{o}\left(\bar{U}_{o ; \varepsilon}\right)\right\|_{\infty}\left\|\varepsilon \psi_{o}\right\|_{L^{2}\left(\mathbb{R} ; \mathbb{R}^{k}\right)} \\
& \quad+\varepsilon \delta\left\|\varepsilon \phi_{o}\right\|_{L^{2}\left(\mathbb{R} ; \mathbb{R}^{n}\right)}+\varepsilon\left\|\varepsilon \theta_{o}\right\|_{L^{2}\left(\mathbb{R} ; \mathbb{R}^{n}\right)} \\
\leq & \left.2 d_{\max }+\varepsilon\left(\tilde{K}_{\mathrm{fam}}+2 \tilde{K}_{F}+\delta_{0}\right)\right]\left\|\mathcal{M}_{\varepsilon}^{1,2} \Phi\right\|_{\mathbf{H}^{1}}+\varepsilon\left\|\mathcal{M}_{\varepsilon}^{1,2} \Theta\right\| .
\end{aligned}
$$

The desired bound hence follows directly from Proposition 4.1.

Copyright (c) by SIAM. Unauthorized reproduction of this article is prohibited. 
The scaling on the second components of $\Phi$ and $\Phi^{\prime}$ can be removed in a similar fashion. However, in this case one also needs to remove the corresponding scaling on $\Theta$.

Corollary 4.4. Consider the setting of Proposition 4.1. Then for each $0<\delta<$ $\delta_{0}$ and $0<\varepsilon<\varepsilon_{0}(\delta)$, the operator $\tilde{\mathcal{L}}_{\varepsilon, \delta}$ satisfies the bound

$$
\left\|\mathcal{M}_{\varepsilon}^{1} \Phi^{\prime}\right\|_{\mathbf{L}^{2}}+\|\Phi\|_{\mathbf{L}^{2}} \leq C_{0}\left[\left\|\mathcal{M}_{\varepsilon}^{1} \Theta\right\|_{\mathbf{L}^{2}}+\frac{1}{\delta}\left|\left\langle\Theta,\left(0, \bar{\Phi}_{e ; 0}^{\mathrm{adj}}\right)\right\rangle_{\mathbf{L}^{2}}\right|\right]
$$

for any $\Phi \in \mathbf{H}^{1}$ and $\Theta=\tilde{\mathcal{L}}_{\varepsilon, \delta} \Phi$, possibly after increasing $C_{0}>0$.

Proof. Writing $\Phi_{o}=\left(\phi_{o}, \psi_{o}\right)$ and $\Theta_{o}=\left(\theta_{o}, \chi_{o}\right)$, we can inspect the definitions (4.7) and (3.12) to obtain

$$
\left(\bar{L}_{o}+\delta\right) \psi_{o}=D_{1} g_{o}\left(\tilde{U}_{o ; \varepsilon}\right) \phi_{o}+\chi_{o}
$$

Using Lemma 3.5 we hence obtain the estimate

$$
\left\|\psi_{o}\right\|_{H^{1}\left(\mathbb{R} ; \mathbb{R}^{k}\right)} \leq C_{1}^{\prime}\left[\left\|D_{1} g_{o}\left(\tilde{U}_{o ; \varepsilon}\right)\right\|_{\infty}\left\|\phi_{o}\right\|_{L^{2}\left(\mathbb{R} ; \mathbb{R}^{n}\right)}+\left\|\chi_{o}\right\|_{L^{2}\left(\mathbb{R} ; \mathbb{R}^{k}\right)}\right]
$$

for some $C_{1}^{\prime}>0$. Combining this with (4.13) yields the desired bound (4.17).

Our final result here provides information on the second derivatives of $\Phi$, in the setting where $\Theta$ is differentiable. In particular, we introduce the spaces

$$
\mathbf{H}_{o}^{2}=\mathbf{H}_{e}^{2}=H^{2}\left(\mathbb{R} ; \mathbb{R}^{n}\right) \times H^{2}\left(\mathbb{R} ; \mathbb{R}^{k}\right), \quad \mathbf{H}^{2}=\mathbf{H}_{o}^{2} \times \mathbf{H}_{e}^{2} .
$$

We remark here that we have chosen to keep the scalings on the second components of $\Phi^{\prime \prime}$ and $\Theta^{\prime}$ because this will be convenient in section 5. Note also that the stated bound on $\|\Phi\|_{\mathbf{H}^{1}}$ can actually be obtained by treating $\tilde{\mathcal{L}}_{\varepsilon, \delta}$ as a regular perturbation of $\mathcal{L}_{\diamond, \delta}$. The point here is that we gain an order of regularity, which is crucial for the nonlinear estimates.

Corollary 4.5. Consider the setting of Proposition 4.1 and assume furthermore that $\left\|\tilde{U}_{\varepsilon}^{\prime}\right\|_{\infty}$ is uniformly bounded for $\varepsilon>0$. Then for each $0<\delta<\delta_{0}$ and any $0<\varepsilon<\varepsilon_{0}(\delta)$, the operator $\tilde{\mathcal{L}}_{\varepsilon, \delta}: \mathbf{H}^{2} \rightarrow \mathbf{H}^{1}$ is invertible and satisfies the bound

$$
\left\|\mathcal{M}_{\varepsilon}^{1,2} \Phi^{\prime \prime}\right\|_{\mathbf{L}^{2}}+\|\Phi\|_{\mathbf{H}^{1}} \leq C_{0}\left[\left\|\mathcal{M}_{\varepsilon}^{1} \Theta\right\|_{\mathbf{L}^{2}}+\left\|\mathcal{M}_{\varepsilon}^{1,2} \Theta^{\prime}\right\|_{\mathbf{L}^{2}}+\frac{1}{\delta}\left|\left\langle\Theta,\left(0, \bar{\Phi}_{e ; 0}^{\mathrm{adj}}\right)\right\rangle_{\mathbf{L}^{2}}\right|\right]
$$

for any $\Phi \in \mathbf{H}^{2}$ and $\Theta=\tilde{\mathcal{L}}_{\varepsilon, \delta} \Phi$, possibly after increasing $C_{0}>0$.

Proof. Pick two constants $0<\delta<\delta_{0}$ and $0<\varepsilon<\varepsilon_{0}(\delta)$ together with a function $\Phi=\left(\Phi_{o}, \Phi_{e}\right) \in \mathbf{H}^{1}$ and write $\Theta=\tilde{\mathcal{L}}_{\varepsilon, \delta} \Phi \in \mathbf{L}^{2}$. If in fact $\Phi \in \mathbf{H}^{2}$, then a direct differentiation shows that

$$
\Theta^{\prime}=\tilde{\mathcal{L}}_{\varepsilon, \delta} \Phi^{\prime}-D^{2} F\left(\tilde{U}_{\varepsilon}\right)\left[\tilde{U}_{\varepsilon}^{\prime}, \Phi\right],
$$

which due to the boundedness of $\Phi$ implies that $\Theta \in \mathbf{H}^{1}$. In particular, $\tilde{\mathcal{L}}_{\varepsilon, \delta}$ maps $\mathbf{H}^{2}$ into $\mathbf{H}^{1}$. Reversely, suppose that we know that $\Theta \in \mathbf{H}^{1}$. Rewriting (4.22) yields

$$
\tilde{c}_{\varepsilon} \Phi^{\prime \prime}=\Theta^{\prime}-\delta \Phi^{\prime}+\mathcal{M}_{1 / \varepsilon^{2}}^{1} J_{\text {mix }} \Phi^{\prime}+D F\left(\tilde{U}_{\varepsilon}\right) \Phi^{\prime}+D^{2} F\left(\tilde{U}_{\varepsilon}\right)\left[\tilde{U}_{\varepsilon}^{\prime}, \Phi\right] .
$$

Since $\Phi$ is bounded, this allows us to conclude that $\Phi \in \mathbf{H}^{2}$. On account of Proposition 4.1 we hence see that $\tilde{\mathcal{L}}_{\varepsilon, \delta}$ is invertible as a map from $\mathbf{H}^{2}$ to $\mathbf{H}^{1}$. 
Fixing $\delta_{\text {ref }}=\frac{1}{2} \delta_{0}$, a short computation shows that

$$
\tilde{\mathcal{L}}_{\varepsilon, \delta_{\text {ref }}} \Phi^{\prime}=\Theta^{\prime}+D^{2} F\left[\tilde{U}_{\varepsilon}^{\prime}, \Phi\right]+\left(\delta_{\text {ref }}-\delta\right) \Phi^{\prime} .
$$

By (4.17) we obtain the bound

$$
\left\|\mathcal{M}_{\varepsilon}^{1} \Phi^{\prime}\right\|_{\mathbf{L}^{2}}+\|\Phi\|_{\mathbf{L}^{2}} \leq C_{0}\left[\left\|\mathcal{M}_{\varepsilon}^{1} \Theta\right\|_{\mathbf{L}^{2}}+\frac{1}{\delta}\left|\left\langle\Theta,\left(0, \bar{\Phi}_{e ; 0}^{\mathrm{adj}}\right)\right\rangle_{\mathbf{L}^{2}}\right|\right] .
$$

On the other hand, (4.13) yields the estimate (4.26)

$$
\begin{aligned}
\left\|\mathcal{M}_{\varepsilon}^{1,2} \Phi^{\prime \prime}\right\|_{\mathbf{L}^{2}}+\left\|\mathcal{M}_{\varepsilon}^{2} \Phi^{\prime}\right\|_{\mathbf{L}^{2}} \leq & C_{0}\left[\left\|\mathcal{M}_{\varepsilon}^{1,2} \Theta^{\prime}\right\|_{\mathbf{L}^{2}}+\left\|\mathcal{M}_{\varepsilon}^{1,2} D^{2} F\left[\tilde{U}_{\varepsilon}^{\prime}, \Phi\right]\right\|_{\mathbf{L}^{2}}\right. \\
& \left.+\left\|\mathcal{M}_{\varepsilon}^{1,2}\left(\delta_{\text {ref }}-\delta\right) \Phi^{\prime}\right\|_{\mathbf{L}^{2}}\right] \\
& +\frac{C_{0}}{\delta_{\text {ref }}}\left|\left\langle\Theta^{\prime}-D^{2} F\left(\tilde{U}_{\varepsilon}\right)\left[\tilde{U}_{\varepsilon}^{\prime}, \Phi\right]-\left(\delta_{\text {ref }}-\delta\right) \Phi^{\prime},\left(0, \bar{\Phi}_{e ; 0}^{\text {adj }}\right)\right\rangle_{\mathbf{L}^{2}}\right|
\end{aligned}
$$

Since $\tilde{U}_{\varepsilon}$ and $\tilde{U}_{\varepsilon}^{\prime}$ are uniformly bounded by assumption, we readily see that

$$
\left\|\mathcal{M}_{\varepsilon}^{1,2} D^{2} F\left(\tilde{U}_{\varepsilon}\right)\left[\tilde{U}_{\varepsilon}^{\prime}, \Phi\right]\right\|_{\mathbf{L}^{2}} \leq\left\|D^{2} F\left(\tilde{U}_{\varepsilon}\right)\left[\tilde{U}_{\varepsilon}^{\prime}, \Phi\right]\right\|_{\mathbf{L}^{2}} \leq C_{1}^{\prime}\|\Phi\|_{\mathbf{L}^{2}}
$$

for some $C_{1}^{\prime}>0$. In particular, we find

$$
\begin{gathered}
\left\|\mathcal{M}_{\varepsilon}^{1,2} \Phi^{\prime \prime}\right\|_{\mathbf{L}^{2}}+\left\|\mathcal{M}_{\varepsilon}^{2} \Phi^{\prime}\right\|_{\mathbf{L}^{2}} \leq C_{2}^{\prime}\left[\left\|\mathcal{M}_{\varepsilon}^{1,2} \Theta^{\prime}\right\|_{\mathbf{L}^{2}}+\|\Phi\|_{\mathbf{L}^{2}}+\left\|\mathcal{M}_{\varepsilon}^{1,2} \Phi^{\prime}\right\|_{\mathbf{L}^{2}}\right. \\
\left.+\left\|\Theta_{e}^{\prime}\right\|_{\mathbf{L}_{e}^{2}}+\left\|\Phi_{e}^{\prime}\right\|_{\mathbf{L}_{e}^{2}}\right]
\end{gathered}
$$

for some $C_{2}^{\prime}>0$. Exploiting the estimates

$$
\left\|\Phi_{e}^{\prime}\right\|_{\mathbf{L}_{e}^{2}} \leq\left\|\mathcal{M}_{\varepsilon}^{1,2} \Phi^{\prime}\right\|_{\mathbf{L}^{2}} \leq\left\|\mathcal{M}_{\varepsilon}^{1} \Phi^{\prime}\right\|_{\mathbf{L}^{2}}, \quad \quad\left\|\Theta_{e}^{\prime}\right\|_{\mathbf{L}_{e}^{2}} \leq\left\|\mathcal{M}_{\varepsilon}^{1,2} \Theta^{\prime}\right\|_{\mathbf{L}^{2}},
$$

together with

$$
\left\|\Phi^{\prime}\right\|_{\mathbf{L}^{2}} \leq\left\|\mathcal{M}_{\varepsilon}^{1} \Phi^{\prime}\right\|_{\mathbf{L}^{2}}+\left\|\mathcal{M}_{\varepsilon}^{2} \Phi^{\prime}\right\|_{\mathbf{L}^{2}},
$$

the bounds (4.25) and (4.28) can be combined to arrive at the desired inequality (4.21).

4.1. Strategy. In this subsection we outline our broad strategy to establish Propositions 4.1 and 4.2. As a first step, we compute the Fredholm index of the operators $\tilde{\mathcal{L}}_{\varepsilon, \lambda}$ for $\lambda$ in a right half-plane that includes the imaginary axis.

LEMma 4.6. Assume that (hFam), (HN1), (HN2), (HW1), and (HW2) are satisfied. Then there exists a constant $\lambda_{0}>0$ so that the operators $\tilde{\mathcal{L}}_{\varepsilon, \lambda}$ are Fredholm with index zero whenever $\operatorname{Re} \lambda \geq-\lambda_{0}$ and $\varepsilon>0$.

Proof. Upon writing

$$
\begin{aligned}
& F_{o ; \rho}^{(1)}=\rho D F_{o}\left(U_{o}^{-}\right)+(1-\rho) D F_{o}\left(U_{o}^{+}\right), \\
& F_{e ; \rho}^{(1)}=\rho D F_{e}\left(U_{e}^{-}\right)+(1-\rho) D F_{e}\left(U_{e}^{+}\right)
\end{aligned}
$$

for any $0 \leq \rho \leq 1$, we introduce the constant coefficient operator $L_{\rho ; \varepsilon, \lambda}: \mathbf{H}_{\mathbb{C}}^{1} \rightarrow \mathbf{L}_{\mathbb{C}}^{2}$ that acts as

$$
L_{\rho ; \varepsilon, \lambda}=\left(\begin{array}{ll}
\tilde{c}_{\varepsilon} \frac{d}{d \xi}+\frac{2}{\varepsilon^{2}} J_{\mathcal{D}}-F_{o ; \rho}^{(1)}+\lambda & -\frac{1}{\varepsilon^{2}} J_{\mathcal{D}} S_{1} \\
-J_{\mathcal{D}} S_{1} & \tilde{c}_{\varepsilon} \frac{d}{d \xi}+2 J_{\mathcal{D}}-F_{e ; \rho}^{(1)}+\lambda
\end{array}\right)
$$

Copyright $@$ by SIAM. Unauthorized reproduction of this article is prohibited. 
and has the associated characteristic function

$$
\Delta_{L_{\rho ; \varepsilon, \lambda}}(z)=\left(\begin{array}{ll}
\tilde{c}_{\varepsilon} z+\frac{2}{\varepsilon^{2}} J_{\mathcal{D}}-F_{o ; \rho}^{(1)}+\lambda & -\frac{1}{\varepsilon^{2}} J_{\mathcal{D}}\left[e^{z}+e^{-z}\right] \\
-J_{\mathcal{D}}\left[e^{z}+e^{-z}\right] & \tilde{c}_{\varepsilon} z+2 J_{\mathcal{D}}-F_{e ; \rho}^{(1)}+\lambda
\end{array}\right) .
$$

Upon writing

$$
F_{\rho}^{(1)}=\left(\begin{array}{ll}
F_{o ; \rho}^{(1)} & 0 \\
0 & F_{e ; \rho}^{(1)}
\end{array}\right)
$$

together with

$$
A(y)=\left(\begin{array}{ll}
J_{\mathcal{D}} & -J_{\mathcal{D}} \cos (y) \\
-J_{\mathcal{D}} \cos (y) & J_{\mathcal{D}}
\end{array}\right)
$$

we see that

$$
\mathcal{M}_{\varepsilon^{2}}^{1,2} \Delta_{L_{\rho ; \varepsilon, \lambda}}(i y)=\left(\tilde{c}_{\varepsilon} i y+\lambda\right) \mathcal{M}_{\varepsilon^{2}}^{1,2}+2 A(y)-\mathcal{M}_{\varepsilon^{2}}^{1,2} F_{\rho}^{(1)} .
$$

For any $y \in \mathbb{R}$ and $V \in \mathbb{C}^{2(n+k)}$ we have

$$
\operatorname{Re} V^{\dagger} \tilde{c}_{\varepsilon} i y \mathcal{M}_{\varepsilon^{2}}^{1,2} V=0
$$

together with

$$
\operatorname{Re} V^{\dagger} A(y) V \geq 0
$$

In particular, we see that

$$
\operatorname{Re} V^{\dagger} \mathcal{M}_{\varepsilon^{2}}^{1,2} \Delta_{L_{\rho ; \varepsilon, \lambda}}(i y) V \geq-\varepsilon^{2} \operatorname{Re}\left[V_{o}^{\dagger}\left(F_{o ; \rho}^{(1)}-\lambda\right) V_{o}\right]-\operatorname{Re}\left[V_{e}^{\dagger}\left(F_{e ; \rho}^{(1)}-\lambda\right) V_{e}\right] .
$$

Let us pick an arbitrary $\lambda_{0}>0$ and suppose that $\Delta_{L_{\rho ; \varepsilon, \lambda}}(i y) V=0$ holds for some $V \in \mathbb{C}^{2(n+k)} \backslash\{0\}$ and $\operatorname{Re} \lambda \geq-\lambda_{0}$. We claim that there exist constants $\vartheta_{1}>0$ and $\vartheta_{2}>0$ that do not depend on $\lambda_{0}$, so that

$$
-\operatorname{Re} V_{\#}^{\dagger}\left(F_{\# ; \rho}^{(1)}-\lambda\right) V_{\#} \geq\left(\vartheta_{2}-\vartheta_{1} \lambda_{0}\right)\left|V_{\#}\right|^{2}
$$

for $\# \in\{o, e\}$. Assuming that this is indeed the case, we pick $\lambda_{0}=\frac{\vartheta_{2}}{2 \vartheta_{1}}$ and obtain the contradiction

$$
\begin{aligned}
0 & =\operatorname{Re} V^{\dagger} \mathcal{M}_{\varepsilon^{2}}^{1,2} \Delta_{L_{\rho ; \varepsilon, \lambda}}(i y) V \\
& \geq \frac{1}{2} \vartheta_{2}\left[\varepsilon^{2}\left|V_{o}\right|^{2}+\left|V_{e}\right|^{2}\right] \\
& >0 .
\end{aligned}
$$

The desired Fredholm properties then follow directly from [39, Thm. C].

In order to establish the claim (4.40), we first assume that $F_{\#}$ satisfies (h $\left.\alpha\right)$. The negative-definiteness of $F_{\# ; \rho}^{(1)}$ then directly yields the bound

$$
\operatorname{Re} V_{\#}^{\dagger}\left(F_{\# ; \rho}^{(1)}-\lambda\right) V_{\#} \leq\left(\lambda_{0}-\vartheta_{2}\right)\left|V_{\#}\right|^{2}
$$

for some $\vartheta_{2}>0$.

Copyright (c) by SIAM. Unauthorized reproduction of this article is prohibited. 
On the other hand, if $F_{\#}$ satisfies $(\mathrm{h} \beta)$, then we can use the identity

$$
\left(\tilde{c}_{\varepsilon} i y+\lambda\right) w_{\#}-\left[F_{\# ; \rho}^{(1)}\right]_{2,2} w_{\#}=\left[F_{\# ; \rho}^{(1)}\right]_{2,1} v_{\#}
$$

to compute

$$
\begin{aligned}
& \operatorname{Re} V_{\#}^{\dagger}\left(\begin{array}{ll}
0 & {\left[F_{\# ; \rho}^{(1)}\right]_{1,2}} \\
{\left[F_{\# ; \rho}^{(1)}\right]_{2,1}} & 0
\end{array}\right) V_{\#} \\
& =\operatorname{Re} V_{\#}^{\dagger}\left(\begin{array}{ll}
0 & -\Gamma\left[F_{\# ; \rho}^{(1)}\right]_{2,1}^{\dagger} \\
{\left[F_{\# ; \rho}^{(1)}\right]_{2,1}} & 0
\end{array}\right) V_{\#} \\
& =\operatorname{Re}\left[-\Gamma v_{\#}^{\dagger}\left[F_{\# ; \rho}^{(1)}\right]_{2,1}^{\dagger} w_{\#}+w_{\#}^{\dagger}\left[F_{\# ; \rho}^{(1)}\right]_{2,1} v_{\#}\right] \\
& =(1-\Gamma) \operatorname{Re} w_{\#}^{\dagger}\left[F_{\# ; \rho}^{(1)}\right]_{2,1} v_{\#} \\
& =(1-\Gamma) \operatorname{Re} w_{\#}^{\dagger}\left[\tilde{c}_{\varepsilon} i y+\lambda\right] w_{\#}-(1-\Gamma) \operatorname{Re} w_{\#}^{\dagger}\left[F_{\# ; \rho}^{(1)}\right]_{2,2} w_{\#} \\
& =(1-\Gamma) \operatorname{Re} \lambda\left|w_{\#}\right|^{2}-(1-\Gamma) \operatorname{Re} w_{\#}^{\dagger}\left[F_{\# ; \rho}^{(1)}\right]_{2,2} w_{\#} \text {. }
\end{aligned}
$$

In particular, Lemma 3.3 allows us to obtain the estimate

$$
\begin{aligned}
\operatorname{Re} V_{\#}^{\dagger}\left(F_{\# ; \rho}^{(1)}-\lambda\right) V_{\#}= & -\Gamma \operatorname{Re} \lambda\left|w_{\#}\right|^{2}+\Gamma \operatorname{Re} w_{\#}^{\dagger}\left[F_{\# ; \rho}^{(1)}\right]_{2,2} w_{\#} \\
& -\operatorname{Re} \lambda\left|v_{\#}\right|^{2}+\operatorname{Re} v_{\#}^{\dagger}\left[F_{\# ; \rho}^{(1)}\right]_{2,2} v_{\#} \\
\leq & (\Gamma+1) \lambda_{0}\left|V_{\#}\right|^{2}-\vartheta_{2}\left|V_{\#}\right|^{2}
\end{aligned}
$$

for some $\vartheta_{2}>0$, as desired.

For any $\varepsilon>0$ and $0<\delta<\delta_{\diamond}$ we introduce the quantity

$$
\Lambda(\varepsilon, \delta)=\inf _{\Phi \in \mathbf{H}^{1},\left\|\mathcal{M}_{\varepsilon}^{1,2} \Phi\right\|_{\mathbf{H}^{1}}=1}\left[\left\|\mathcal{M}_{\varepsilon}^{1,2} \tilde{\mathcal{L}}_{\varepsilon, \delta} \Phi\right\|_{\mathbf{L}^{2}}+\frac{1}{\delta}\left|\left\langle\tilde{\mathcal{L}}_{\varepsilon, \delta} \Phi,\left(0, \bar{\Phi}_{e ; 0}^{\mathrm{adj}}\right)\right\rangle_{\mathbf{L}^{2}}\right|\right],
$$

which allows us to define

$$
\Lambda(\delta)=\liminf _{\varepsilon \downarrow 0} \Lambda(\varepsilon, \delta) .
$$

Similarly, for any $\varepsilon>0$ and any subset $M \subset \mathbb{C}$ we write

$$
\Lambda(\varepsilon, M)=\inf _{\Phi \in \mathbf{H}^{1}, \lambda \in M,\left\|\mathcal{M}_{\varepsilon}^{1,2} \Phi\right\|_{\mathbf{H}^{1}}=1}\left\|\mathcal{M}_{\varepsilon}^{1,2} \tilde{\mathcal{L}}_{\varepsilon, \lambda} \Phi\right\|_{\mathbf{L}^{2}}
$$

together with

$$
\Lambda(M)=\liminf _{\varepsilon \downarrow 0} \Lambda(\varepsilon, M) .
$$

The following proposition forms the key ingredient for proving Propositions 4.1 and 4.2. It is the analogue of [1, Lem. 6].

Proposition 4.7. Assume that (hFam), (HN1), (HN2), (HW1), (HW2), and (HS1) are satisfied. Then there exist constants $\delta_{0}>0$ and $C_{0}>0$ so that

$$
\Lambda(\delta) \geq \frac{2}{C_{0}}
$$

holds for all $0<\delta<\delta_{0}$.

Assume furthermore that (HS2) holds and pick a sufficiently small $\lambda_{0}>0$. Then for any subset $M \subset \mathbb{C}$ that satisfies $\left(h M_{\lambda_{0}}\right)$, there exists a constant $C_{M}$ so that

$$
\Lambda(M) \geq \frac{2}{C_{M}} .
$$

Copyright $@$ by SIAM. Unauthorized reproduction of this article is prohibited. 
Proof of Proposition 4.1. Fix $0<\delta<\delta_{0}$. Proposition 4.7 implies that we can pick $\varepsilon_{0}(\delta)>0$ in such a way that $\Lambda(\varepsilon, \delta) \geq \frac{1}{C_{0}}$ for each $0<\varepsilon<\varepsilon_{0}(\delta)$. This means that $\tilde{\mathcal{L}}_{\varepsilon, \delta}$ is injective for each such $\varepsilon$ and that the bound (4.11) holds for any $\Phi \in \mathbf{H}^{1}$. Since $\tilde{\mathcal{L}}_{\varepsilon, \delta}$ is also a Fredholm operator with index zero by Lemma 4.6, it must be invertible.

Proof of Proposition 4.2. The result can be established by repeating the arguments used in the proof of Proposition 4.1.

4.2. Proof of Proposition 4.7. We now set out to prove Proposition 4.7. In Lemmas 4.8 and 4.9 we construct weakly converging sequences that realize the infima in (4.46)-(4.49). In Lemmas 4.10-4.15 we exploit the structure of our operator (4.10) to recover lower bounds on the norms of the derivatives of these sequences that are typically lost when taking weak limits. First recall the constant $\delta_{\diamond}$ from Proposition 3.1.

Lemma 4.8. Consider the setting of Proposition 4.7 and pick $0<\delta<\delta$. Then there exists a sequence

$$
\left\{\left(\varepsilon_{j}, \Phi_{j}, \Theta_{j}\right)\right\}_{j \geq 1} \subset(0,1) \times \mathbf{H}^{1} \times \mathbf{L}^{2}
$$

together with a pair of functions

$$
\Phi \in \mathbf{H}^{1}, \quad \Theta \in \mathbf{L}^{2}
$$

that satisfy the following properties:

(i) We have $\lim _{j \rightarrow \infty} \varepsilon_{j}=0$ together with

$$
\lim _{j \rightarrow \infty}\left[\left\|\mathcal{M}_{\varepsilon_{j}}^{1,2} \Theta_{j}\right\|_{\mathbf{L}^{2}}+\frac{1}{\delta}\left|\left\langle\Theta_{j},\left(0, \bar{\Phi}_{e ; 0}^{\mathrm{adj}}\right)\right\rangle_{\mathbf{L}^{2}}\right|\right]=\Lambda(\delta) .
$$

(ii) For every $j \geq 1$ we have the identity

$$
\tilde{\mathcal{L}}_{\varepsilon_{j}, \delta} \Phi_{j}=\Theta_{j}
$$

together with the normalization

$$
\left\|\mathcal{M}_{\varepsilon_{j}}^{1,2} \Phi_{j}\right\|_{\mathbf{H}^{1}}=1 .
$$

(iii) Writing $\Phi=\left(\phi_{o}, \psi_{o}, \phi_{e}, \psi_{e}\right)$, we have $\phi_{o}=0$.

(iv) The sequence $\mathcal{M}_{\varepsilon_{j}}^{1,2} \Phi_{j}$ converges to $\Phi$ strongly in $\mathbf{L}_{\text {loc }}^{2}$ and weakly in $\mathbf{H}^{1}$. In addition, the sequence $\mathcal{M}_{\varepsilon_{j}}^{1,2} \Theta_{j}$ converges weakly to $\Theta$ in $\mathbf{L}^{2}$.

Proof. Items (i) and (ii) follow directly from the definition of $\Lambda(\delta)$. The normalization (4.56) and the limit (4.54) ensure that $\left\|\mathcal{M}_{\varepsilon_{j}}^{1,2} \Phi_{j}\right\|_{\mathbf{H}^{1}}$ and $\left\|\mathcal{M}_{\varepsilon_{j}}^{1,2} \Theta_{j}\right\|_{\mathbf{L}^{2}}$ are bounded, which allows us to obtain the weak limits (iv) after passing to a subsequence.

In order to obtain (iii), we write $\Phi_{j}=\left(\phi_{o, j}, \psi_{o, j}, \phi_{e, j}, \psi_{e, j}\right)$ together with $\Theta_{j}=$ $\left(\theta_{o, j}, \chi_{o, j}, \theta_{e, j}, \chi_{e, j}\right)$ and note that the first component of (4.55) yields

$$
\begin{aligned}
2 \mathcal{D} \phi_{o, j}-\mathcal{D} S_{1} \phi_{e, j}= & -\varepsilon_{j}^{2} \tilde{c}_{\varepsilon_{j}} \phi_{o, j}^{\prime}+\varepsilon_{j}^{2} D_{1} f_{o}\left(\tilde{U}_{o ; \varepsilon_{j}}\right) \phi_{o, j} \\
& +\varepsilon_{j}^{2} D_{2} f_{o}\left(\tilde{U}_{o ; \varepsilon_{j}}\right) \psi_{o, j}-\delta \varepsilon_{j}^{2} \phi_{o, j}+\varepsilon_{j}^{2} \theta_{o, j} .
\end{aligned}
$$

The normalization condition (4.56) and the limit (4.54) hence imply that

$$
\lim _{j \rightarrow \infty}\left\|2 \mathcal{D} \phi_{o ; j}-\mathcal{D} S_{1} \phi_{e, j}\right\|_{L^{2}\left(\mathbb{R} ; \mathbb{R}^{n}\right)}=0 .
$$

In particular, we see that $\left\{\phi_{o ; j}\right\}_{j \geq 1}$ is a bounded sequence. This yields the desired identity $\phi_{o}=\lim _{j \rightarrow \infty} \varepsilon_{j} \phi_{o, j}=0$. 
Lemma 4.9. Consider the setting of Proposition 4.7 and pick a sufficiently small $\lambda_{0}>0$. Then for any $M \subset \mathbb{C}$ that satisfies $\left(h M_{\lambda_{0}}\right)$, there exists a sequence

$$
\left\{\left(\lambda_{j}, \varepsilon_{j}, \Phi_{j}, \Theta_{j}\right)\right\}_{j \geq 1} \subset M \times(0,1) \times \mathbf{H}^{1} \times \mathbf{L}^{2}
$$

together with a triplet

$$
\Phi \in \mathbf{H}^{1}, \quad \Theta \in \mathbf{L}^{2}, \quad \lambda \in M
$$

that satisfy the limits

$$
\varepsilon_{j} \rightarrow 0, \quad \lambda_{j} \rightarrow \lambda, \quad\left\|\mathcal{M}_{\varepsilon_{j}}^{1,2} \Theta_{j}\right\|_{\mathbf{L}^{2}} \rightarrow \Lambda(M)
$$

as $j \rightarrow \infty$, together with the properties (ii)-(iv) from Lemma 4.8, with $\delta$ replaced by $\lambda_{j}$ in (4.55).

Proof. These properties can be obtained by following the proof of Lemma 4.8 in an almost identical fashion.

In the remainder of this section we will often treat the settings of Lemmas 4.8 and 4.9 in a parallel fashion. In order to streamline our notation, we use the value $\lambda_{0}$ stated in Lemma 4.6 and interpret $\left\{\lambda_{j}\right\}_{j \geq 1}$ as the constant sequence $\lambda_{j}=\delta$ when working in the context of Lemma 4.8. In addition, we write $\lambda_{\max }=\delta_{\diamond}$ in the setting of Lemma 4.8 or $\lambda_{\max }=\max \{|\lambda|: \lambda \in M\}$ in the setting of Lemma 4.9.

Lemma 4.10. Consider the setting of 4.8 or 4.9 . Then the function $\Phi$ from Lemma 4.8 satisfies

$$
\|\Phi\|_{\mathbf{H}^{1}} \leq C_{\diamond} \Lambda(\delta),
$$

while the function $\Phi$ from Lemma 4.9 satisfies

$$
\|\Phi\|_{\mathbf{H}^{1}} \leq C_{\diamond ; M} \Lambda(M) .
$$

Proof. In order to take the $\varepsilon \downarrow 0$ limit in a controlled fashion, we introduce the operator

$$
\tilde{L}_{0 ; \lambda}=\lim _{j \rightarrow \infty} \mathcal{M}_{\varepsilon_{j}^{2}}^{1} \tilde{\mathcal{L}}_{\varepsilon_{j}, \lambda_{j}}
$$

Upon introducing the top-left block

$$
\left[\tilde{L}_{0 ; \lambda}\right]_{1,1}=\left(\begin{array}{cc}
2 \mathcal{D} & 0 \\
-D_{1} g_{o}\left(\bar{U}_{o ; 0}\right) & \bar{L}_{o}+\lambda
\end{array}\right),
$$

we can explicitly write

$$
\tilde{L}_{0 ; \lambda}=\left(\begin{array}{ll}
{\left[\tilde{L}_{0 ; \lambda}\right]_{1,1}} & -J \mathcal{D} S_{1} \\
-J \mathcal{D} S_{1} & c_{0} \frac{d}{d \xi}+2 J \mathcal{D}-D F_{e}\left(\bar{U}_{e ; 0}\right)+\lambda
\end{array}\right) .
$$

Note that $\tilde{L}_{0 ; \lambda}$ and its adjoint $\tilde{L}_{0 ; \lambda}^{\text {adj }}$ are both bounded operators from $\mathbf{H}^{1}$ to $\mathbf{L}^{2}$.

In addition, we introduce the commutators

$$
B_{j}=\tilde{\mathcal{L}}_{\varepsilon_{j}, \lambda_{j}} M_{\varepsilon_{j}}^{1,2}-M_{\varepsilon_{j}}^{1,2} \tilde{\mathcal{L}}_{\varepsilon_{j}, \lambda_{j}} .
$$

Copyright (c) by SIAM. Unauthorized reproduction of this article is prohibited. 
A short computation shows that

$$
B_{j}=\left(\begin{array}{cc}
{\left[B_{j}\right]_{1,1}} & \left(\frac{1}{\varepsilon_{j}}-\frac{1}{\varepsilon_{j}^{2}}\right) J_{\mathcal{D}} S_{1} \\
\left(1-\varepsilon_{j}\right) J_{\mathcal{D}} S_{1} & 0
\end{array}\right),
$$

in which the top-left block is given by

$$
\left[B_{j}\right]_{1,1}=\left(1-\varepsilon_{j}\right)\left(\begin{array}{cc}
0 & D_{2} f_{o}\left(\tilde{U}_{o ; \varepsilon_{j}}\right) \\
-D_{1} g_{o}\left(\tilde{U}_{o ; \varepsilon_{j}}\right) & 0
\end{array}\right) .
$$

Pick any test-function $Z \in C^{\infty}\left(\mathbb{R} ; \mathbb{R}^{2 n+2 k}\right)$ and write

$$
\mathcal{I}_{j}=\left\langle\mathcal{M}_{\varepsilon_{j}^{2}}^{1} \tilde{\mathcal{L}}_{\varepsilon_{j}, \lambda_{j}} \mathcal{M}_{\varepsilon_{j}}^{1,2} \Phi_{j}, Z\right\rangle_{\mathbf{L}^{2}} .
$$

Using the strong convergence

$$
\tilde{\mathcal{L}}_{\varepsilon_{j}, \lambda_{j}}^{\text {adj }} \mathcal{M}_{\varepsilon_{j}^{2}}^{1} Z \rightarrow \tilde{L}_{0 ; \lambda}^{\mathrm{adj}} Z \in \mathbf{L}^{2},
$$

we obtain the limit

$$
\begin{aligned}
\mathcal{I}_{j} & =\left\langle\mathcal{M}_{\varepsilon_{j}}^{1,2} \Phi_{j}, \tilde{\mathcal{L}}_{\varepsilon_{j}, \lambda_{j}}^{\text {adj }} \mathcal{M}_{\varepsilon_{j}^{2}}^{1} Z\right\rangle_{\mathbf{L}^{2}} \\
& \rightarrow\left\langle\Phi, \tilde{L}_{0 ; \lambda}^{\text {adj }} Z\right\rangle_{\mathbf{L}^{2}} \\
& =\left\langle\tilde{L}_{0 ; \lambda} \Phi, Z\right\rangle_{\mathbf{L}^{2}}
\end{aligned}
$$

as $j \rightarrow \infty$.

In particular, we see that

$$
\begin{aligned}
\mathcal{I}_{j} & =\left\langle\mathcal{M}_{\varepsilon_{j}^{2}}^{1} \mathcal{M}_{\varepsilon_{j}}^{1,2} \tilde{\mathcal{L}}_{\varepsilon_{j}, \lambda_{j}} \Phi_{j}, Z\right\rangle_{\mathbf{L}^{2}}+\left\langle\mathcal{M}_{\varepsilon_{j}^{2}}^{1} B_{j} \Phi_{j}, Z\right\rangle_{\mathbf{L}^{2}} \\
& =\left\langle\mathcal{M}_{\varepsilon_{j}^{2}}^{1} \mathcal{M}_{\varepsilon_{j}}^{1,2} \Theta_{j}, Z\right\rangle_{\mathbf{L}^{2}}+\left\langle\mathcal{M}_{\varepsilon_{j}^{2}}^{1} B_{j} \Phi_{j}, Z\right\rangle_{\mathbf{L}^{2}} \\
& \rightarrow\left\langle\mathcal{M}_{0}^{1} \Theta, Z\right\rangle_{\mathbf{L}^{2}}+\left\langle\left(-\mathcal{D} S_{1} \phi_{e},-D_{1} g_{o}\left(\bar{U}_{o ; 0}\right) \phi_{o}, \mathcal{D} S_{1} \phi_{o}, 0\right), Z\right\rangle_{\mathbf{L}^{2}} .
\end{aligned}
$$

It hence follows that

$$
\tilde{L}_{0 ; \delta} \Phi=\mathcal{M}_{0}^{1} \Theta+\left(-\mathcal{D} S_{1} \phi_{e},-D_{1} g_{o}\left(\bar{U}_{o ; 0}\right) \phi_{o}, \mathcal{D} S_{1} \phi_{o}, 0\right) .
$$

Introducing the functions

$$
\Phi_{\diamond}=\left(\psi_{0}, \phi_{e}, \psi_{e}\right), \quad \Theta_{\diamond}=\left(\chi_{o}, \theta_{e}, \chi_{e}\right),
$$

the identity $\phi_{o}=0$ implies that

$$
\mathcal{L}_{\diamond, \lambda} \Phi_{\diamond}=\Theta_{\diamond}
$$

In the setting of Lemma 4.8, we may hence use Proposition 3.1 to compute

$$
\begin{aligned}
\left\|\Phi_{\diamond}\right\|_{\mathbf{H}_{\diamond}^{1}} & \leq C_{\diamond}\left[\left\|\Theta_{\diamond}\right\|_{\mathbf{L}_{\diamond}^{2}}+\frac{1}{\delta}\left|\left\langle\Theta_{\diamond},\left(0, \bar{\Phi}_{e ; 0}^{\mathrm{adj}}\right)\right\rangle_{\mathbf{L}_{\diamond}^{2}}\right|\right] \\
& \leq C_{\diamond}\left[\|\Theta\|_{\mathbf{L}^{2}}+\frac{1}{\delta}\left|\left\langle\Theta,\left(0, \bar{\Phi}_{e ; 0}^{\mathrm{adj}}\right)\right\rangle_{\mathbf{L}^{2}}\right|\right] .
\end{aligned}
$$

The lower semicontinuity of the $L^{2}$-norm and the convergence in (iv) of Lemma 4.8 
imply that

$$
\|\Theta\|_{\mathbf{L}^{2}}+\frac{1}{\delta}\left|\left\langle\Theta,\left(0, \bar{\Phi}_{e ; 0}^{\mathrm{adj}}\right)\right\rangle_{\mathbf{L}^{2}}\right| \leq \Lambda(\delta) .
$$

In particular, we find

$$
\|\Phi\|_{\mathbf{H}^{1}}=\left\|\Phi_{\diamond}\right\|_{\mathbf{H}_{\diamond}^{1}} \leq C_{\diamond} \Lambda(\delta),
$$

as desired. In the setting of Lemma 4.9 the bound follows in a similar fashion.

We note that

$$
\mathcal{M}_{\varepsilon_{j}^{2}}^{1,2} \Theta_{j}=\tilde{c}_{\varepsilon_{j}} \mathcal{M}_{\varepsilon_{j}^{2}}^{1,2} \Phi_{j}^{\prime}+\mathcal{M}_{\varepsilon_{j}^{2}}^{1,2}\left(-D F\left(\tilde{U}_{\varepsilon_{j}}\right)+\lambda_{j}\right) \Phi_{j}-J_{\mathrm{mix}} \Phi_{j},
$$

in which $J_{\text {mix }}$ is given by (4.9) and in which

$$
D F\left(\tilde{U}_{\varepsilon}\right)=\left(\begin{array}{ll}
D F_{o}\left(\tilde{U}_{o ; \varepsilon}\right) & 0 \\
0 D F_{e}\left(\tilde{U}_{e ; \varepsilon}\right) &
\end{array}\right)
$$

Lemma 4.11. Assume that (HN1) is satisfied. Then the bounds

$$
\begin{aligned}
\operatorname{Re}\left\langle-J_{\text {mix }} \Phi, \Phi^{\prime}\right\rangle_{\mathbf{L}^{2}} & =0, \\
\operatorname{Re}\left\langle-J_{\text {mix }} \Phi, \Phi\right\rangle_{\mathbf{L}^{2}} & \geq 0
\end{aligned}
$$

hold for all $\Phi \in \mathbf{H}_{\mathbb{C}}^{1}$.

Proof. Pick $\Phi \in \mathbf{H}_{\mathbb{C}}^{1}$ and write $\Phi=\left(\Phi_{o}, \Phi_{e}\right)$. We can compute

$$
\begin{aligned}
\operatorname{Re}\left\langle-J_{\text {mix }} \Phi, \Phi^{\prime}\right\rangle_{\mathbf{L}^{2}}= & \operatorname{Re}\left\langle 2 J_{\mathcal{D}} \Phi_{o}, \Phi_{o}^{\prime}\right\rangle_{\mathbf{L}_{o}^{2}}-\operatorname{Re}\left\langle J_{\mathcal{D}} S_{1} \Phi_{e}, \Phi_{o}^{\prime}\right\rangle_{\mathbf{L}_{o}^{2}} \\
& \quad-\operatorname{Re}\left\langle J_{\mathcal{D}} S_{1} \Phi_{o}, \Phi_{e}^{\prime}\right\rangle_{\mathbf{L}_{e}^{2}}+2 \operatorname{Re}\left\langle J_{\mathcal{D}} \Phi_{e}, \Phi_{e}^{\prime}\right\rangle_{\mathbf{L}_{e}^{2}} \\
= & 0,
\end{aligned}
$$

since we have $\operatorname{Re}\left\langle J_{\mathcal{D}} S_{1} \Phi_{e}, \Phi_{o}^{\prime}\right\rangle_{\mathbf{L}_{o}^{2}}=-\operatorname{Re}\left\langle J_{\mathcal{D}} S_{1} \Phi_{o}, \Phi_{e}^{\prime}\right\rangle_{\mathbf{L}_{e}^{2}}$. Moreover, we can estimate $(4.84)$

$$
\begin{aligned}
\operatorname{Re}\left\langle-J_{\text {mix }} \Phi, \Phi\right\rangle_{\mathbf{L}^{2}}= & \operatorname{Re}\left\langle 2 J_{\mathcal{D}} \Phi_{o}, \Phi_{o}\right\rangle_{\mathbf{L}_{o}^{2}}-\operatorname{Re}\left\langle J_{\mathcal{D}} S_{1} \Phi_{e}, \Phi_{o}\right\rangle_{\mathbf{L}_{o}^{2}} \\
& \quad-\operatorname{Re}\left\langle J_{\mathcal{D}} S_{1} \Phi_{o}, \Phi_{e}\right\rangle_{\mathbf{L}_{e}^{2}}+2 \operatorname{Re}\left\langle J_{\mathcal{D}} \Phi_{e}, \Phi_{e}\right\rangle_{\mathbf{L}_{e}^{2}} \\
\geq & 2\left\|\sqrt{J_{\mathcal{D}}} \Phi_{o}\right\|_{\mathbf{L}_{o}^{2}}^{2}+2\left\|\sqrt{J_{\mathcal{D}}} \Phi_{e}\right\|_{\mathbf{L}_{e}^{2}}^{2}-4\left\|\sqrt{J_{\mathcal{D}}} \Phi_{o}\right\|_{\mathbf{L}_{o}^{2}}\left\|\sqrt{J_{\mathcal{D}}} \Phi_{e}\right\|_{\mathbf{L}_{e}^{2}} \\
\geq & 2\left\|\sqrt{J_{\mathcal{D}}} \Phi_{o}\right\|_{\mathbf{L}_{o}^{2}}^{2}+2\left\|\sqrt{J_{\mathcal{D}}} \Phi_{e}\right\|_{\mathbf{L}_{e}^{2}}^{2}-4\left(\frac{1}{2}\left\|\sqrt{J_{\mathcal{D}}} \Phi_{o}\right\|_{\mathbf{L}_{o}^{2}}^{2}+\frac{1}{2}\left\|\sqrt{J_{\mathcal{D}}} \Phi_{e}\right\|_{\mathbf{L}_{e}^{2}}^{2}\right) \\
= & 0 .
\end{aligned}
$$

Lemma 4.12. Consider the setting of Lemma 4.8 or 4.9. Then the bound $(4.85)$

$\left|\operatorname{Re}\left\langle\mathcal{M}_{\varepsilon_{j}^{2}}^{1,2}\left(-D F\left(\tilde{U}_{\varepsilon_{j}}\right)+\lambda_{j}\right) \Phi_{j}, \Phi_{j}^{\prime}\right\rangle_{\mathbf{L}^{2}}\right| \leq 2\left(\tilde{K}_{F}+\lambda_{\max }\right)\left\|\mathcal{M}_{\varepsilon_{j}}^{1,2} \Phi\right\|_{\mathbf{L}^{2}}\left\|\mathcal{M}_{\varepsilon_{j}}^{1,2} \Phi_{j}^{\prime}\right\|_{\mathbf{L}^{2}}$

holds for all $j \geq 1$.

Proof. We first note that (4.86)

$$
\begin{aligned}
\operatorname{Re}\left\langle\mathcal{M}_{\varepsilon_{j}^{2}}^{1,2}\left(-D F\left(\tilde{U}_{\varepsilon_{j}}\right)+\lambda_{j}\right) \Phi_{j}, \Phi_{j}^{\prime}\right\rangle_{\mathbf{L}^{2}}= & \operatorname{Re}\left\langle\varepsilon_{j}\left(-D F_{o}\left(\tilde{U}_{o ; \varepsilon_{j}}\right)+\lambda_{j}\right) \Phi_{o, j}, \varepsilon_{j} \Phi_{o, j}^{\prime}\right\rangle_{\mathbf{L}_{o}^{2}} \\
& +\operatorname{Re}\left\langle\left(-D F_{e}\left(\tilde{U}_{e ; \varepsilon_{j}}\right)+\lambda_{j}\right) \Phi_{e, j}, \Phi_{e, j}^{\prime}\right\rangle_{\mathbf{L}_{e}^{2}}
\end{aligned}
$$

Copyright $@$ ( ) by SIAM. Unauthorized reproduction of this article is prohibited. 
Using Cauchy-Schwarz we compute (4.87)

$$
\begin{aligned}
\left|\operatorname{Re}\left\langle\mathcal{M}_{\varepsilon_{j}^{2}}^{1,2}\left(-D F\left(\tilde{U}_{\varepsilon_{j}}\right)+\lambda_{j}\right) \Phi_{j}, \Phi_{j}^{\prime}\right\rangle_{\mathbf{L}^{2}}\right| \leq & \left(\tilde{K}_{F}+\lambda_{\max }\right)\left\|\varepsilon_{j} \Phi_{o, j}\right\|_{\mathbf{L}_{o}^{2}}\left\|\varepsilon_{j} \Phi_{o, j}^{\prime}\right\|_{\mathbf{L}_{o}^{2}} \\
& +\left(\tilde{K}_{F}+\lambda_{\max }\right)\left\|\Phi_{e, j}\right\|_{\mathbf{L}_{e}^{2}}\left\|\Phi_{e, j}^{\prime}\right\|_{\mathbf{L}_{e}^{2}} \\
\leq & 2\left(\tilde{K}_{F}+\lambda_{\max }\right)\left\|\mathcal{M}_{\varepsilon_{j}}^{1,2} \Phi_{j}\right\|_{\mathbf{L}^{2}}\left\|\mathcal{M}_{\varepsilon_{j}}^{1,2} \Phi_{j}^{\prime}\right\|_{\mathbf{L}^{2}}
\end{aligned}
$$

as desired.

Lemma 4.13. Consider the setting of Lemma 4.8 or 4.9 , possibly decreasing the size of $\lambda_{0}>0$. Then there exist strictly positive constants $(a, m, g)$ together with a constant $\beta \geq 0$ so that the bound

$$
\begin{gathered}
\operatorname{Re}\left\langle\mathcal{M}_{\varepsilon_{j}^{2}}^{1,2}\left(-D F\left(\tilde{U}_{\varepsilon_{j}}\right)+\lambda_{j}\right) \Phi_{j}, \Phi_{j}\right\rangle_{\mathbf{L}^{2}} \geq a\left\|\mathcal{M}_{\varepsilon_{j}}^{1,2} \Phi_{j}\right\|_{\mathbf{L}^{2}}^{2}-g \int_{|x| \leq m}\left|\mathcal{M}_{\varepsilon_{j}}^{1,2} \Phi_{j}\right|^{2} \\
-\beta\left\|\mathcal{M}_{\varepsilon_{j}}^{1,2} \Theta_{j}\right\|_{\mathbf{L}^{2}}^{2}
\end{gathered}
$$

holds for all $j \geq 1$.

Proof. We first note that

$$
\operatorname{Re}\left\langle\mathcal{M}_{\varepsilon_{j}^{2}}^{1,2}\left(-D F\left(\tilde{U}_{\varepsilon_{j}}\right)+\lambda_{j}\right) \Phi_{j}, \Phi_{j}\right\rangle_{\mathbf{L}^{2}}=\varepsilon^{2} \mathcal{N}_{o ; j}+\mathcal{N}_{e ; j}
$$

in which we have defined

$$
\mathcal{N}_{\#, j}=\operatorname{Re}\left\langle\left(-D F_{\#}\left(\tilde{U}_{\# ; \varepsilon_{j}}\right)+\lambda_{j}\right) \Phi_{\#, j}, \Phi_{\#, j}\right\rangle_{\mathbf{L}_{\#}^{2}}
$$

for $\# \in\{o, e\}$.

Let us first suppose that $F_{\#}$ satisfies $(\mathrm{h} \beta)$ and let $\Gamma_{\#}$ be the proportionality constant from that assumption. We start by studying the cross-term

$$
\begin{aligned}
\mathcal{C}_{\#, j}=- & \operatorname{Re}\left\langle D_{2} f_{\#}\left(\tilde{U}_{\# ; \varepsilon_{j}}\right) \psi_{\#, j}, \phi_{\#, j}\right\rangle_{L^{2}\left(\mathbb{R} ; \mathbb{R}^{n}\right)} \\
& -\operatorname{Re}\left\langle D_{1} g_{\#}\left(\tilde{U}_{\# ; \varepsilon_{j}}\right) \phi_{\#, j}, \psi_{\#, j}\right\rangle_{L^{2}\left(\mathbb{R} ; \mathbb{R}^{k}\right.} .
\end{aligned}
$$

Recalling that

$$
\chi_{\#, j}=\tilde{c}_{\varepsilon_{j}} \psi_{\#, j}^{\prime}-D g_{\# ; 1}\left(\tilde{U}_{\# ; \varepsilon_{j}}\right) \phi_{\#, j}-D g_{\# ; 2}\left(\tilde{U}_{\# ; \varepsilon_{j}}\right) \psi_{\#, j}+\lambda_{j} \psi_{\#, j},
$$

we obtain the identity

$$
\begin{aligned}
\mathcal{C}_{\#, j}= & \left(\Gamma_{\#}-1\right) \operatorname{Re}\left\langle D_{1} g_{\#}\left(\tilde{U}_{\# ; \varepsilon_{j}}\right) \phi_{\#, j}, \psi_{\#, j}\right\rangle_{L^{2}\left(\mathbb{R} ; \mathbb{R}^{k}\right)} \\
= & \left(\Gamma_{\#}-1\right) \operatorname{Re}\left\langle\tilde{c}_{\varepsilon_{j}} \psi_{\#, j}^{\prime}-D_{2} g_{\#}\left(\tilde{U}_{\# ; \varepsilon_{j}}\right) \psi_{\#, j}+\lambda_{j} \psi_{\#, j}-\chi_{\#, j}, \psi_{\#, j}\right\rangle_{L^{2}\left(\mathbb{R} ; \mathbb{R}^{k}\right)} \\
= & \tilde{c}_{\varepsilon_{j}}\left(\Gamma_{\#}-1\right) \operatorname{Re}\left\langle\psi_{\#, j}^{\prime}, \psi_{\#, j}\right\rangle_{L^{2}\left(\mathbb{R} ; \mathbb{R}^{k}\right)} \\
& \quad+\left(\Gamma_{\#}-1\right) \operatorname{Re}\left\langle-D_{2} g_{\#}\left(\tilde{U}_{\# ; \varepsilon_{j}}\right) \psi_{\#, j}+\lambda_{j} \psi_{\#, j}-\chi_{\#, j}, \psi_{\#, j}\right\rangle_{L^{2}\left(\mathbb{R} ; \mathbb{R}^{k}\right)} \\
= & \left(1-\Gamma_{\#}\right) \operatorname{Re}\left\langle D_{2} g_{\#}\left(\tilde{U}_{\# ; \varepsilon_{j}}\right) \psi_{\#, j}, \psi_{\#, j}\right\rangle_{L^{2}\left(\mathbb{R} ; \mathbb{R}^{k}\right)} \\
& \quad+\left(\Gamma_{\#}-1\right)\left[\operatorname{Re} \lambda\left\|\psi_{\#, j}\right\|_{\mathbf{L}_{\#}}^{2}-\left\langle\chi_{\#, j}, \psi_{\#, j}\right\rangle_{\left.L^{2}\left(\mathbb{R} ; \mathbb{R}^{k}\right)\right] .}\right.
\end{aligned}
$$

In particular, we see that

$$
\begin{aligned}
\mathcal{N}_{\#, j}=\Gamma_{\#} & \operatorname{Re} \lambda\left\langle\psi_{\#, j}, \psi_{\#, j}\right\rangle_{L^{2}\left(\mathbb{R} ; \mathbb{R}^{k}\right)}-\Gamma_{\#} \operatorname{Re}\left\langle D_{2} g_{\#}\left(\tilde{U}_{\# ; \varepsilon_{j}}\right) \psi_{\#, j}, \psi_{\#, j}\right\rangle_{L^{2}\left(\mathbb{R} ; \mathbb{R}^{k}\right)} \\
& +\operatorname{Re} \lambda\left\langle\phi_{\#, j}, \phi_{\#, j}\right\rangle_{L^{2}\left(\mathbb{R} ; \mathbb{R}^{n}\right)}-\operatorname{Re}\left\langle D_{1} f_{\#}\left(\tilde{U}_{\# ; \varepsilon_{j}}\right) \phi_{\#, j}, \phi_{\#, j}\right\rangle_{L^{2}\left(\mathbb{R} ; \mathbb{R}^{n}\right)} \\
& -\left(\Gamma_{\#}-1\right)\left\langle\chi_{\#, j}, \psi_{\#, j}\right\rangle_{L^{2}\left(\mathbb{R} ; \mathbb{R}^{k}\right)}
\end{aligned}
$$

Copyright $@$ by SIAM. Unauthorized reproduction of this article is prohibited. 
Recall that $\tilde{U}_{\varepsilon} \rightarrow \bar{U}_{0}$ in $L^{\infty}, \tilde{U}_{o ; \varepsilon_{j}}(\xi) \rightarrow U_{o}^{ \pm}$, and $\tilde{U}_{e ; \varepsilon_{j}}(\xi) \rightarrow U_{e}^{ \pm}$for $\xi \rightarrow \pm \infty$. Using Lemma 3.3 and decreasing $\lambda_{0}$ if necessary, we see that there exist $a>\left(\Gamma_{\#}+\right.$ 1) $\lambda_{0}>0$ and $m \gg 1$ so that

$$
\begin{aligned}
3 a\left|\Phi_{\#, j}(\xi)\right|^{2} \leq- & \operatorname{Re}\left\langle D_{1} f_{\#}\left(\tilde{U}_{\# ; \varepsilon_{j}}(\xi)\right) \phi_{\#, j}(\xi), \phi_{\#, j}(\xi)\right\rangle_{\mathbb{R}^{n}} \\
& -\Gamma_{\#} \operatorname{Re}\left\langle D_{2} g_{\#}\left(\tilde{U}_{\# ; \varepsilon_{j}}(\xi)\right) \psi_{\#, j}(\xi), \psi_{\#, j}(\xi)\right\rangle_{\mathbb{R}^{k}}
\end{aligned}
$$

for all $|\xi| \geq m$. We hence obtain

$$
\begin{gathered}
\mathcal{N}_{\#, j} \geq 2 a \int_{|\xi| \geq m}\left|\Phi_{\#, j}(\xi)\right|^{2} d \xi-\left(\Gamma_{\#}+1\right)\left(\tilde{K}_{F}+\lambda_{\max }\right) \int_{|\xi| \leq m}\left|\Phi_{\#, j}(\xi)\right|^{2} d \xi \\
-\left(\Gamma_{\#}+1\right)\left\|\chi_{\#, j}\right\|_{L^{2}\left(\mathbb{R} ; \mathbb{R}^{k}\right)}\left\|\psi_{\#, j}\right\|_{L^{2}\left(\mathbb{R} ; \mathbb{R}^{k}\right)} \\
\geq 2 a\left\|\Phi_{\#, j}\right\|_{\mathbf{L}_{\#}^{2}}^{2}-\left(\Gamma_{\#}+1\right)\left(2 a+\tilde{K}_{F}+\lambda_{\max }\right) \int_{|\xi| \leq m}\left|\Phi_{\#, j}(\xi)\right|^{2} d \xi \\
\quad-\left(\Gamma_{\#}+1\right)\left\|\chi_{\#, j}\right\|_{L^{2}\left(\mathbb{R} ; \mathbb{R}^{k}\right)}\left\|\psi_{\#, j}\right\|_{L^{2}\left(\mathbb{R} ; \mathbb{R}^{k}\right)} .
\end{gathered}
$$

Using the standard identity $x y \leq \frac{1}{4 z} x^{2}+z y^{2}$ for $x, y \in \mathbb{R}$ and $z>0$, we now find

$$
\begin{gathered}
\mathcal{N}_{\#, j} \geq a\left\|\Phi_{\#, j}\right\|_{\mathbf{L}_{\#}^{2}}^{2}-\left(\Gamma_{\#}+1\right)\left(2 a+\tilde{K}_{F}+\lambda_{\max }\right) \int_{|\xi| \leq m}\left|\Phi_{\#, j}(\xi)\right|^{2} d \xi \\
-\frac{1}{4 a}\left(\Gamma_{\#}+1\right)^{2}\left\|\chi_{\#, j}\right\|_{L^{2}\left(\mathbb{R} ; \mathbb{R}^{k}\right)}^{2},
\end{gathered}
$$

which has the desired form.

In the case where $F_{\#}$ satisfies $(\mathrm{h} \alpha)$, a similar bound can be obtained in an analogous, but far easier fashion.

Lemma 4.14. Consider the setting of Lemma 4.8 or 4.9. Then there exists a constant $\kappa>0$ so that the bound

$$
\kappa\left\|\mathcal{M}_{\varepsilon_{j}}^{1,2} \Phi_{j}\right\|_{\mathbf{L}^{2}}^{2} \geq\left\|\mathcal{M}_{\varepsilon_{j}}^{1,2} \Phi_{j}^{\prime}\right\|_{\mathbf{L}^{2}}^{2}-2 \tilde{K}_{\text {fam }}^{2}\left\|\mathcal{M}_{\varepsilon_{j}}^{1,2} \Theta_{j}\right\|_{\mathbf{L}^{2}}^{2}
$$

holds for all $j \geq 1$.

Proof. For convenience, we assume that $\tilde{c}_{\varepsilon_{j}}>0$ for all $j \geq 1$. Recalling the decomposition (4.80), we can use Lemmas 4.11 and 4.12 to compute (4.99)

$$
\begin{aligned}
\operatorname{Re}\left\langle\mathcal{M}_{\varepsilon_{j}}^{1,2} \Theta_{j}, \mathcal{M}_{\varepsilon_{j}}^{1,2} \Phi_{j}^{\prime}\right\rangle_{\mathbf{L}^{2}}= & \tilde{c}_{\varepsilon_{j}} \operatorname{Re}\left\langle\mathcal{M}_{\varepsilon_{j}}^{1,2} \Phi_{j}^{\prime}, \mathcal{M}_{\varepsilon_{j}}^{1,2} \Phi_{j}^{\prime}\right\rangle_{\mathbf{L}^{2}}+\operatorname{Re}\left\langle-J_{\text {mix }} \Phi_{j}, \Phi_{j}^{\prime}\right\rangle_{\mathbf{L}^{2}} \\
& +\operatorname{Re}\left\langle\mathcal{M}_{\varepsilon_{j}^{2}}^{1,2}\left(-D F\left(\tilde{U}_{\varepsilon_{j}}\right)+\lambda_{j}\right) \Phi_{j}, \Phi_{j}^{\prime}\right\rangle_{\mathbf{L}^{2}} \\
\geq & -2\left(\tilde{K}_{F}+\lambda_{\max }\right)\left\|\mathcal{M}_{\varepsilon_{j}}^{1,2} \Phi_{j}\right\|_{\mathbf{L}^{2}}\left\|\mathcal{M}_{\varepsilon_{j}}^{1,2} \Phi_{j}^{\prime}\right\|_{\mathbf{L}^{2}}+\tilde{c}_{\varepsilon_{j}}\left\|\mathcal{M}_{\varepsilon_{j}}^{1,2} \Phi_{j}^{\prime}\right\|_{\mathbf{L}^{2}}^{2} .
\end{aligned}
$$

We hence see that

$$
\tilde{c}_{\varepsilon_{j}}\left\|\mathcal{M}_{\varepsilon_{j}}^{1,2} \Phi_{j}^{\prime}\right\|_{\mathbf{L}^{2}}^{2} \leq 2\left(\tilde{K}_{F}+\lambda_{\max }\right)\left\|\mathcal{M}_{\varepsilon_{j}}^{1,2} \Phi_{j}\right\|_{\mathbf{L}^{2}}\left\|\mathcal{M}_{\varepsilon_{j}}^{1,2} \Phi_{j}^{\prime}\right\|_{\mathbf{L}^{2}}+\left\|\mathcal{M}_{\varepsilon_{j}}^{1,2} \Theta_{j}\right\|_{\mathbf{L}^{2}}\left\|\mathcal{M}_{\varepsilon_{j}}^{1,2} \Phi_{j}^{\prime}\right\|_{\mathbf{L}^{2}} .
$$

Dividing by $\left\|\mathcal{M}_{\varepsilon_{j}}^{1,2} \Phi_{j}^{\prime}\right\|_{\mathbf{L}^{2}}$ and squaring, we find

$$
\tilde{c}_{\varepsilon_{j}}^{2}\left\|\mathcal{M}_{\varepsilon_{j}}^{1,2} \Phi_{j}^{\prime}\right\|_{\mathbf{L}^{2}}^{2} \leq 8\left(\tilde{K}_{F}+\lambda_{\max }\right)^{2}\left\|\mathcal{M}_{\varepsilon_{j}}^{1,2} \Phi_{j}\right\|_{\mathbf{L}^{2}}^{2}+2\left\|\mathcal{M}_{\varepsilon_{j}}^{1,2} \Theta_{j}\right\|_{\mathbf{L}^{2}}^{2},
$$

as desired.

Recall the constants $(g, m, a, \beta)$ introduced in Lemma 4.13. Throughout the remainder of this section, we set out to obtain a lower bound for the integral

$$
\mathcal{I}_{j}=g \int_{|\xi| \leq m}\left|\mathcal{M}_{\varepsilon_{j}}^{1,2} \Phi_{j}(\xi)\right|^{2} d \xi .
$$

Copyright $@$ by SIAM. Unauthorized reproduction of this article is prohibited. 
Lemma 4.15. Consider the setting of Lemma 4.8 or 4.9 . Then the bound

$$
\mathcal{I}_{j} \geq \frac{a}{2}\left\|\mathcal{M}_{\varepsilon_{j}}^{1,2} \Phi_{j}\right\|_{\mathbf{L}^{2}}^{2}-\left(\frac{1}{2 a}+\beta\right)\left\|\mathcal{M}_{\varepsilon_{j}}^{1,2} \Theta_{j}\right\|_{\mathbf{L}^{2}}^{2}
$$

holds for all $j \geq 1$.

Proof. Recall the decomposition (4.80). Combining the estimates in Lemmas 4.11 and 4.13 and remembering that $\operatorname{Re}\left\langle\mathcal{M}_{\varepsilon_{j}}^{1,2} \Phi_{j}^{\prime}, \mathcal{M}_{\varepsilon_{j}}^{1,2} \Phi_{j}\right\rangle_{\mathbf{L}^{2}}=0$, we find

$$
\begin{aligned}
\mathcal{I}_{j} & \geq a\left\|\mathcal{M}_{\varepsilon_{j}}^{1,2} \Phi_{j}\right\|_{\mathbf{L}^{2}}^{2}-\operatorname{Re}\left\langle\mathcal{M}_{\varepsilon_{j}}^{1,2} \Theta_{j}, \mathcal{M}_{\varepsilon_{j}}^{1,2} \Phi_{j}\right\rangle_{\mathbf{L}^{2}}-\beta\left\|\mathcal{M}_{\varepsilon_{j}}^{1,2} \Theta_{j}\right\|_{\mathbf{L}^{2}}^{2} \\
& \geq a\left\|\mathcal{M}_{\varepsilon_{j}}^{1,2} \Phi_{j}\right\|_{\mathbf{L}^{2}}^{2}-\left\|\mathcal{M}_{\varepsilon_{j}}^{1,2} \Theta_{j}\right\|_{\mathbf{L}^{2}}\left\|\mathcal{M}_{\varepsilon_{j}}^{1,2} \Phi_{j}\right\|_{\mathbf{L}^{2}}-\beta\left\|\mathcal{M}_{\varepsilon_{j}}^{1,2} \Theta_{j}\right\|_{\mathbf{L}^{2}}^{2} .
\end{aligned}
$$

Using the standard identity $x y \leq \frac{z}{2} x^{2}+\frac{1}{2 z} y^{2}$ for $x, y \in \mathbb{R}$ and $z>0$ we can estimate

$$
\mathcal{I}_{j} \geq \frac{a}{2}\left\|\mathcal{M}_{\varepsilon_{j}}^{1,2} \Phi_{j}\right\|_{\mathbf{L}^{2}}^{2}-\left(\frac{1}{2 a}+\beta\right)\left\|\mathcal{M}_{\varepsilon_{j}}^{1,2} \Theta_{j}\right\|_{\mathbf{L}^{2}}^{2}
$$

as desired.

Proof of Proposition 4.7. Introducing the constant $\gamma=\frac{a}{2(\kappa+1)}$, we add $\gamma$ times (4.98) to (4.103) and find

$$
\begin{aligned}
\mathcal{I}_{j}+\frac{a \kappa}{2(\kappa+1)}\left\|\mathcal{M}_{\varepsilon_{j}}^{1,2} \Phi_{j}\right\|_{\mathbf{L}^{2}}^{2} \geq & \frac{a}{2}\left\|\mathcal{M}_{\varepsilon_{j}}^{1,2} \Phi_{j}\right\|_{\mathbf{L}^{2}}^{2}-\left(\frac{1}{2 a}+\beta\right)\left\|\mathcal{M}_{\varepsilon_{j}}^{1,2} \Theta_{j}\right\|_{\mathbf{L}^{2}}^{2} \\
& +\frac{a}{2(\kappa+1)}\left\|\mathcal{M}_{\varepsilon_{j}}^{1,2} \Phi_{j}^{\prime}\right\|_{\mathbf{L}^{2}}-\frac{a \tilde{K}_{\text {fam }}^{2}}{2(\kappa+1)}\left\|\mathcal{M}_{\varepsilon_{j}}^{1,2} \Theta_{j}\right\|_{\mathbf{L}^{2}}^{2} .
\end{aligned}
$$

We hence obtain

$$
\begin{aligned}
\mathcal{I}_{j} & \geq \frac{a}{2(\kappa+1)}\left\|\mathcal{M}_{\varepsilon_{j}}^{1,2} \Phi_{j}\right\|_{\mathbf{H}^{1}}-\left(\frac{1}{2 a}+\beta+\frac{a \tilde{K}_{\text {fam }}^{2}}{2(\kappa+1)}\right)\left\|\mathcal{M}_{\varepsilon_{j}}^{1,2} \Theta_{j}\right\|_{\mathbf{L}^{2}}^{2} \\
& :=C_{1}-C_{2}\left\|\mathcal{M}_{\varepsilon_{j}}^{1,2} \Theta_{j}\right\|_{\mathbf{L}^{2}}^{2} .
\end{aligned}
$$

Letting $j \rightarrow \infty$ in the setting of Lemma 4.8 yields

$$
C_{1}-C_{2} \Lambda(\delta) \leq g \int_{|\xi| \leq m}|\Phi(\xi)|^{2} d \xi \leq g C_{\diamond} \Lambda(\delta)
$$

As such, we can conclude that

$$
\Lambda(\delta) \geq \frac{2}{C_{0}}
$$

for some $C_{0}>0$, as required. An analogous computation can be used for the setting of Lemma 4.9 .

5. Existence of traveling waves. In this section we follow the spirit of $[1$, Thm. 1] and develop a fixed point argument to show that (2.1) admits traveling wave solutions of the form (2.12). The main complication is that we need $\varepsilon$-uniform bounds on the supremum norm of the waveprofiles in order to control the nonlinear terms. This can be achieved by bounding the $\mathbf{H}^{1}$-norm of the perturbation, but the estimates in Proposition 4.1 feature a problematic scaling factor on the odd component. Fortunately Corollary 4.5 does provide uniform $\mathbf{H}^{1}$-bounds, but it requires us to take a derivative of the traveling wave system.

Throughout this section we will apply the results from section 4 to the constant family

$$
\left(\tilde{U}_{\varepsilon}, \tilde{c}_{\varepsilon}\right)=\left(\bar{U}_{0}, c_{0}\right)
$$

Copyright $@$ by SIAM. Unauthorized reproduction of this article is prohibited. 
which clearly satisfies (hFam). In particular, we fix a small constant $\delta>0$ and write $\mathcal{L}_{\varepsilon, \delta}$ for the operators given by (4.7) in this setting. We set out to construct a branch of wavespeeds $c_{\varepsilon}$ and small functions

$$
\Phi_{\varepsilon}=\left(\Phi_{o ; \varepsilon}, \Phi_{e ; \varepsilon}\right) \in \mathbf{H}^{2}
$$

in such a way that $\bar{U}_{0}+\Phi_{\varepsilon}$ is a solution to (2.14). A short computation shows that this is equivalent to the system

$$
\mathcal{L}_{\varepsilon, \delta}\left(\Phi_{\varepsilon}\right)=\mathcal{F}_{\delta}\left(c_{\varepsilon}, \Phi_{\varepsilon}\right),
$$

which we split up by introducing the expressions

$$
\begin{aligned}
\mathcal{R}(c, \Phi) & =\left(c_{0}-c\right) \partial_{\xi}\left(\bar{U}_{0}+\Phi\right), \\
\mathcal{E}_{0} & =\left(-J c_{0} \bar{U}_{o ; 0}^{\prime}+J F_{o}\left(\bar{U}_{o ; 0}\right), 0\right), \\
\mathcal{N}_{\#}\left(\Phi_{\#}\right) & =F_{\#}\left(\bar{U}_{\# ; 0}+\Phi_{\#}\right)-D F_{\#}\left(\bar{U}_{\# ; 0}\right) \Phi_{\#}-F_{\#}\left(\bar{U}_{\# ; 0}\right)
\end{aligned}
$$

for $\# \in\{o, e\}$ and writing

$$
\mathcal{F}_{\delta}\left(c_{\varepsilon}, \Phi_{\varepsilon}\right)=\mathcal{R}\left(c_{\varepsilon}, \Phi_{\varepsilon}\right)+\mathcal{E}_{0}+\left(\mathcal{N}_{o}\left(\Phi_{o ; \varepsilon}\right), \mathcal{N}_{e}\left(\Phi_{e ; \varepsilon}\right)\right)+\delta \Phi .
$$

Notice that $\mathcal{R}$ contains a derivative of $\Phi$. It is hence crucial that $\mathcal{L}_{\varepsilon, \delta}^{-1}$ gains an order of regularity, which we obtained by the framework developed in section 4 .

For any $\varepsilon>0$ and $\Phi \in \mathbf{H}^{2}$ we introduce the norm

$$
\|\Phi\|_{\mathbf{X}_{\varepsilon}}^{2}=\left\|\mathcal{M}_{\varepsilon}^{1,2} \partial_{\xi}^{2} \Phi\right\|_{\mathbf{L}^{2}}^{2}+\|\Phi\|_{\mathbf{H}^{1}}^{2},
$$

which is equivalent to the standard norm on $\mathbf{H}^{2}$. For any $\eta>0$, this allows us to introduce the set

$$
\mathbf{X}_{\eta ; \varepsilon}=\left\{\Phi \in \mathbf{H}^{2}:\|\Phi\|_{\mathbf{X}_{\varepsilon}} \leq \eta\right\}
$$

For convenience, we introduce the constant $\eta_{*}=\left[2\left\|\Phi_{e ; 0}^{\text {adj }}\right\|_{\mathbf{L}_{e}^{2}}\right]^{-1}$, together with the formal expression

$$
c_{\delta}\left(\Phi_{e}\right)=c_{0}+\left[1+\left\langle\partial_{\xi} \Phi_{e}, \bar{\Phi}_{e ; 0}^{\mathrm{adj}}\right\rangle_{\mathbf{L}_{e}^{2}}\right]^{-1}\left[\delta\left\langle\Phi_{e}, \bar{\Phi}_{e ; 0}^{\mathrm{adj}}\right\rangle_{\mathbf{L}_{e}^{2}}+\left\langle\mathcal{N}_{e}\left(\Phi_{e}\right), \bar{\Phi}_{e ; 0}^{\mathrm{adj}}\right\rangle_{\mathbf{L}_{e}^{2}}\right] .
$$

Lemma 5.1. Assume that (HN1), (HN2), (HW1), (HW2), and (HS1) are satisfied and pick a constant $0<\eta \leq \eta_{*}$. Then the expression (5.8) is well-defined for any $\varepsilon>0$ and any $\Phi=\left(\Phi_{o}, \Phi_{e}\right) \in \mathbf{X}_{\eta ; \varepsilon}$. In addition, the equation

$$
\left\langle\mathcal{F}_{\delta}(c, \Phi),\left(0, \bar{\Phi}_{e ; 0}^{\mathrm{adj}}\right)\right\rangle_{\mathbf{L}^{2}}=0
$$

has the unique solution $c=c_{\delta}\left(\Phi_{e}\right)$.

Proof. We first note that

$$
\left\langle\partial_{\xi} \Phi_{e}, \bar{\Phi}_{e ; 0}^{\mathrm{adj}}\right\rangle_{\mathbf{L}_{e}^{2}} \geq-\left\|\partial_{\xi} \Phi_{e}\right\|_{\mathbf{L}_{e}^{2}}\left\|\bar{\Phi}_{e ; 0}^{\mathrm{adj}}\right\|_{\mathbf{L}_{e}^{2}} \geq-\frac{1}{2}
$$

Copyright $@$ by SIAM. Unauthorized reproduction of this article is prohibited. 
which implies that (5.8) is well-defined. The result now follows by noting that $\left\langle\mathcal{E}_{0},\left(0, \bar{\Phi}_{e ; 0}^{\mathrm{adj}}\right)\right\rangle_{\mathbf{L}^{2}}=0$ and that

$$
\begin{aligned}
\left\langle\mathcal{R}(c, \Phi),\left(0, \bar{\Phi}_{e ; 0}^{\mathrm{adj}}\right)\right\rangle_{\mathbf{L}^{2}} & =\left(c_{0}-c\right)\left(\left\langle\bar{U}_{0 ; e}^{\prime}, \bar{\Phi}_{e ; 0}^{\mathrm{adj}}\right\rangle_{\mathbf{L}_{e}^{2}}+\left\langle\partial_{\xi} \Phi_{e}, \bar{\Phi}_{e ; 0}^{\mathrm{adj}}\right\rangle_{\mathbf{L}_{e}^{2}}\right) \\
& =\left(c_{0}-c\right)\left(1+\left\langle\partial_{\xi} \Phi_{e}, \bar{\Phi}_{e ; 0}^{\mathrm{adj}}\right\rangle_{\mathbf{L}_{e}^{2}}\right)
\end{aligned}
$$

which implies that

$\left\langle\mathcal{F}_{\delta}(c, \Phi),\left(0, \bar{\Phi}_{e ; 0}^{\mathrm{adj}}\right)\right\rangle_{\mathbf{L}^{2}}=\left(c_{0}-c\right)\left(1+\left\langle\partial_{\xi} \Phi_{e}, \bar{\Phi}_{e ; 0}^{\mathrm{adj}}\right\rangle_{\mathbf{L}_{e}^{2}}\right)+\delta\left\langle\Phi_{e}, \bar{\Phi}_{e ; 0}^{\mathrm{adj}}\right\rangle_{\mathbf{L}_{e}^{2}}+\left\langle\mathcal{N}_{e}\left(\Phi_{e}\right), \bar{\Phi}_{e ; 0}^{\mathrm{adj}}\right\rangle_{\mathbf{L}_{e}^{2}}$.

Consider the setting of Corollary 4.5 and pick $0<\delta<\delta_{0}$ and $0<\varepsilon<\varepsilon_{0}(\delta)$. Our goal here is to find solutions to (5.3) by showing that the map $T_{\varepsilon, \delta}: \mathbf{X}_{\eta ; \varepsilon} \rightarrow \mathbf{H}^{2}$ that acts as

$$
T_{\varepsilon, \delta}(\Phi)=\left(\mathcal{L}_{\varepsilon, \delta}\right)^{-1} \mathcal{F}_{\delta}\left(c_{\delta}\left(\Phi_{e}\right), \Phi\right)
$$

admits a fixed point. For any triplet $\left(\Phi, \Phi^{A}, \Phi^{B}\right) \in \mathbf{X}_{\eta ; \varepsilon}^{3}$, the bounds in Corollary 4.5 imply that

$$
\left\|T_{\varepsilon, \delta}(\Phi)\right\|_{\mathbf{X}_{\varepsilon}} \leq C_{0}\left[\left\|\mathcal{M}_{\varepsilon}^{1} \mathcal{F}_{\delta}\left(c_{\delta}\left(\Phi_{e}\right), \Phi\right)\right\|_{\mathbf{L}^{2}}+\left\|\mathcal{M}_{\varepsilon}^{1,2} \partial_{\xi} \mathcal{F}_{\delta}\left(c_{\delta}\left(\Phi_{e}\right), \Phi\right)\right\|_{\mathbf{L}^{2}}\right]
$$

together with

$$
\begin{aligned}
\left\|T_{\varepsilon, \delta}\left(\Phi^{A}\right)-T_{\varepsilon, \delta}\left(\Phi^{B}\right)\right\|_{\mathbf{X}_{\varepsilon} \leq} & C_{0}\left\|\mathcal{M}_{\varepsilon}^{1}\left(\mathcal{F}_{\delta}\left(c_{\delta}\left(\Phi_{e}^{A}\right), \Phi^{A}\right)-\mathcal{F}_{\delta}\left(c_{\delta}\left(\Phi_{e}^{B}\right), \Phi^{B}\right)\right)\right\|_{\mathbf{L}^{2}} \\
& +C_{0}\left\|\mathcal{M}_{\varepsilon}^{1,2} \partial_{\xi}\left(\mathcal{F}_{\delta}\left(c_{\delta}\left(\Phi_{e}^{A}\right), \Phi^{A}\right)-\mathcal{F}_{\delta}\left(c_{\delta}\left(\Phi_{e}^{B}\right), \Phi^{B}\right)\right)\right\|_{\mathbf{L}^{2}} .
\end{aligned}
$$

In order to show that $T_{\varepsilon, \delta}$ is a contraction mapping, it hence suffices to obtain suitable bounds for the terms appearing on the right-hand side of these estimates.

We start by obtaining pointwise bounds on the nonlinear terms. To this end, we compute

$$
\begin{aligned}
\partial_{\xi} \mathcal{N}_{o}\left(\Phi_{o}\right)=( & \left.D F_{o}\left(\bar{U}_{o ; 0}+\Phi_{o}\right)-D F_{o}\left(\bar{U}_{o ; 0}\right)-D^{2} F_{o}\left(\bar{U}_{o ; 0}\right) \Phi_{o}\right) \bar{U}_{o ; 0}^{\prime} \\
& +\left(D F_{o}\left(\bar{U}_{o ; 0}+\Phi_{o}\right)-D F_{o}\left(\bar{U}_{o ; 0}\right)\right) \partial_{\xi} \Phi_{o}
\end{aligned}
$$

and note that a similar identity holds for $\partial_{\xi} \mathcal{N}_{e}\left(\Phi_{e}\right)$. In addition, we remark that there is a constant $K_{F}>0$ for which the bounds

$$
\left\|D F_{\#}\left(\bar{U}_{\# ; 0}+\Phi_{\#}\right)\right\|_{\infty}+\left\|D^{2} F_{\#}\left(\bar{U}_{\# ; 0}+\Phi_{\#}\right)\right\|_{\infty}+\left\|D^{3} F_{\#}\left(\bar{U}_{\# ; 0}+\Phi_{\#}\right)\right\|_{\infty}<K_{F}
$$

hold for $\# \in\{o, e\}$ and all $\Phi=\left(\Phi_{o}, \Phi_{e}\right)$ that have $\|\Phi\|_{\mathbf{H}^{1}} \leq \eta_{*}$.

Lemma 5.2. Assume that (HN1), (HN2), (HW1), and (HW2) are satisfied. There exists a constant $K_{p}>0$ so that for each $\Phi=\left(\Phi_{o}, \Phi_{e}\right) \in \mathbf{H}^{1}$ with $\|\Phi\|_{\mathbf{H}^{1}} \leq \eta_{*}$, we have the pointwise estimates

$$
\begin{aligned}
& \left|\mathcal{N}_{o}\left(\Phi_{o}\right)\right| \leq K_{p}\left|\Phi_{o}\right|^{2}, \\
& \left|\mathcal{N}_{e}\left(\Phi_{e}\right)\right| \leq K_{p}\left|\Phi_{e}\right|^{2} .
\end{aligned}
$$

Copyright $@$ by SIAM. Unauthorized reproduction of this article is prohibited. 
Proof. Using [17, Thm. 2.8.3] we obtain

$$
\left|\mathcal{N}_{o}\left(\Phi_{o}\right)\right| \leq \frac{1}{2} K_{F}\left|\Phi_{o}\right|^{2} .
$$

The estimate for $\mathcal{N}_{e}$ follows similarly.

Lemma 5.3. Assume that (HN1), (HN2), (HW1), and (HW2) are satisfied. There exists a constant $K_{p}>0$ so that for each $\Phi=\left(\Phi_{o}, \Phi_{e}\right) \in \mathbf{H}^{1}$ with $\|\Phi\|_{\mathbf{H}^{1}} \leq \eta_{*}$, we have the pointwise estimates

$$
\begin{aligned}
& \left|\partial_{\xi} \mathcal{N}_{o}\left(\Phi_{o}\right)\right| \leq K_{p}\left(\left|\partial_{\xi} \Phi_{o}\right|\left|\Phi_{o}\right|+\left|\Phi_{o}\right|^{2}\right), \\
& \left|\partial_{\xi} \mathcal{N}_{e}\left(\Phi_{e}\right)\right| \leq K_{p}\left(\left|\partial_{\xi} \Phi_{e}\right|\left|\Phi_{e}\right|+\left|\Phi_{e}\right|^{2}\right) .
\end{aligned}
$$

Proof. We rewrite (5.16) to obtain

$$
\begin{aligned}
\partial_{\xi} \mathcal{N}_{o}\left(\Phi_{o}\right)=D F_{o}\left(\bar{U}_{o ; 0}+\Phi_{o}\right) \partial_{\xi}\left(\bar{U}_{o ; 0}+\Phi_{o}\right)-D F_{o}\left(\bar{U}_{o ; 0}\right) \partial_{\xi}\left(\bar{U}_{o ; 0}+\Phi_{o}\right) \\
-D^{2} F_{o}\left(\bar{U}_{o ; 0}\right)\left[\Phi_{o}, \partial_{\xi}\left(\bar{U}_{o ; 0}+\Phi_{o}\right)\right]+D^{2} F_{o}\left(\bar{U}_{o ; 0}\right)\left[\Phi_{o}, \partial_{\xi} \Phi_{o}\right] .
\end{aligned}
$$

This allows us to use [17, Thm. 2.8.3] and obtain the pointwise estimate

$$
\begin{aligned}
\left|\partial_{\xi} \mathcal{N}_{o}\left(\Phi_{o}\right)\right| & \leq \frac{1}{2} K_{F}\left|\Phi_{o}\right|^{2}\left(\left|\bar{U}_{o ; 0}^{\prime}\right|+\left|\partial_{\xi} \Phi_{o}\right|\right)+K_{F}\left|\Phi_{o}\right|\left|\partial_{\xi} \Phi_{o}\right| \\
& \leq K_{p}\left(\left|\partial_{\xi} \Phi_{o}\right|\left|\Phi_{o}\right|+\left|\Phi_{o}\right|^{2}\right) .
\end{aligned}
$$

The estimate for $\mathcal{N}_{e}$ follows similarly.

Lemma 5.4. Assume that (HN1), (HN2), (HW1), and (HW2) are satisfied. There exists a constant $K_{p}>0$ so that for each pair

$$
\Phi^{A}=\left(\Phi_{o}^{A}, \Phi_{e}^{A}\right) \in \mathbf{H}^{1}, \quad \Phi^{B}=\left(\Phi_{o}^{B}, \Phi_{e}^{B}\right) \in \mathbf{H}^{1}
$$

that satisfies $\left\|\Phi^{A}\right\|_{\mathbf{H}^{1}} \leq \eta_{*}$ and $\left\|\Phi^{B}\right\|_{\mathbf{H}^{1}} \leq \eta_{*}$, we have the pointwise estimates

$$
\begin{aligned}
& \left|\mathcal{N}_{o}\left(\Phi_{o}^{A}\right)-\mathcal{N}_{o}\left(\Phi_{o}^{B}\right)\right| \leq K_{p}\left[\left|\Phi_{o}^{A}\right|+\left|\Phi_{o}^{B}\right|\right]\left|\Phi_{o}^{A}-\Phi_{o}^{B}\right|, \\
& \left|\mathcal{N}_{e}\left(\Phi_{e}^{A}\right)-\mathcal{N}_{e}\left(\Phi_{e}^{B}\right)\right| \leq K_{p}\left[\left|\Phi_{e}^{A}\right|+\left|\Phi_{e}^{B}\right|\right]\left|\Phi_{e}^{A}-\Phi_{e}^{B}\right| .
\end{aligned}
$$

Proof. We first compute

$$
\begin{aligned}
\mathcal{N}_{o}\left(\Phi_{o}^{A}\right)-\mathcal{N}_{o}\left(\Phi_{o}^{B}\right)=F_{o} & \left.\bar{U}_{o ; 0}+\Phi_{o}^{B}+\left(\Phi_{o}^{A}-\Phi_{o}^{B}\right)\right)-F_{o}\left(\bar{U}_{o ; 0}+\Phi_{o}^{B}\right) \\
& -D F_{o}\left(\bar{U}_{o ; 0}+\Phi_{o}^{B}\right)\left(\Phi_{o}^{A}-\Phi_{o}^{B}\right) \\
& +\left[D F_{o}\left(\bar{U}_{o ; 0}+\Phi_{o}^{B}\right)-D F_{o}\left(\bar{U}_{o ; 0}\right)\right]\left(\Phi_{o}^{A}-\Phi_{o}^{B}\right) .
\end{aligned}
$$

Applying [17, Thm. 2.8.3] twice yields the pointwise estimate

$$
\begin{aligned}
\left|\mathcal{N}_{o}\left(\Phi_{o}^{A}\right)-\mathcal{N}_{o}\left(\Phi_{o}^{B}\right)\right| & \leq K_{F}\left[\frac{1}{2}\left|\Phi_{o}^{A}-\Phi_{o}^{B}\right|^{2}+\left|\Phi_{o}^{B}\right|\left|\Phi_{o}^{A}-\Phi_{o}^{B}\right|\right] \\
& \leq 2 K_{F}\left[\left|\Phi_{o}^{A}\right|+\left|\Phi_{o}^{B}\right|\right]\left|\Phi_{o}^{A}-\Phi_{o}^{B}\right| .
\end{aligned}
$$

The estimate for $\mathcal{N}_{e}$ follows similarly.

Lemma 5.5. Assume that (HN1), (HN2), (HW1), and (HW2) are satisfied. There exists a constant $K_{p}>0$ so that for each pair

$$
\Phi^{A}=\left(\Phi_{o}^{A}, \Phi_{e}^{A}\right) \in \mathbf{H}^{1}, \quad \Phi^{B}=\left(\Phi_{o}^{B}, \Phi_{e}^{B}\right) \in \mathbf{H}^{1}
$$

Copyright $@$ by SIAM. Unauthorized reproduction of this article is prohibited. 
that satisfies $\left\|\Phi^{A}\right\|_{\mathbf{H}^{1}} \leq \eta_{*}$ and $\left\|\Phi^{B}\right\|_{\mathbf{H}^{1}} \leq \eta_{*}$ we have the pointwise estimates $(5.28)$

$$
\begin{aligned}
\left|\partial_{\xi} \mathcal{N}_{\#}\left(\Phi_{\#}^{A}\right)-\partial_{\xi} \mathcal{N}_{\#}\left(\Phi_{\#}^{B}\right)\right| \leq K_{p}\left[\left|\partial_{\xi} \Phi_{\#}^{A}\right|+\left|\Phi_{\#}^{A}\right|+\left|\partial_{\xi} \Phi_{\#}^{B}\right|+\left|\Phi_{\#}^{B}\right|\right]\left|\Phi_{\#}^{A}-\Phi_{\#}^{B}\right| \\
+K_{p}\left[\left|\Phi_{\#}^{A}\right|+\left|\Phi_{\#}^{B}\right|\right]\left|\partial_{\xi}\left(\Phi_{\#}^{A}-\Phi_{\#}^{B}\right)\right|
\end{aligned}
$$

for $\# \in\{o, e\}$.

Proof. Differentiating (5.25) line by line, we obtain

$$
\partial_{\xi} \mathcal{N}_{o}\left(\Phi_{o}^{A}\right)-\partial_{\xi} \mathcal{N}_{o}\left(\Phi_{o}^{B}\right)=d_{1}+d_{2}+d_{3}
$$

with

$$
\begin{aligned}
d_{1}= & D F_{o}\left(\bar{U}_{o ; 0}+\Phi_{o}^{B}+\left(\Phi_{o}^{A}-\Phi_{o}^{B}\right)\right)\left(\bar{U}_{o ; 0}^{\prime}+\partial_{\xi} \Phi_{o}^{B}+\partial_{\xi}\left(\Phi_{o}^{A}-\Phi_{o}^{B}\right)\right) \\
& \quad-D F_{o}\left(\bar{U}_{o ; 0}+\Phi_{o}^{B}\right) \partial_{\xi}\left(\bar{U}_{o ; 0}+\Phi_{o}^{B}\right), \\
d_{2}=- & D^{2} F_{o}\left(\bar{U}_{o ; 0}+\Phi_{o}^{B}\right)\left[\Phi_{o}^{A}-\Phi_{o}^{B}, \partial_{\xi}\left(\bar{U}_{o ; 0}+\Phi_{o}^{B}\right)\right]-D F_{o}\left(\bar{U}_{o ; 0}+\Phi_{o}^{B}\right) \partial_{\xi}\left(\Phi_{o}^{A}-\Phi_{o}^{B}\right), \\
d_{3}=[ & \left.D F_{o}\left(\bar{U}_{o ; 0}+\Phi_{o}^{B}\right)-D F_{o}\left(\bar{U}_{o ; 0}\right)\right] \partial_{\xi}\left(\Phi_{o}^{A}-\Phi_{o}^{B}\right) \\
& \quad+D^{2} F_{o}\left(\bar{U}_{o ; 0}+\Phi_{o}^{B}\right)\left[\partial_{\xi}\left(\bar{U}_{o ; 0}+\Phi_{o}^{B}\right), \Phi_{o}^{A}-\Phi_{o}^{B}\right]-D^{2} F_{o}\left(\bar{U}_{o ; 0}\right)\left[\bar{U}_{o ; 0}^{\prime}, \Phi_{o}^{A}-\Phi_{o}^{B}\right] .
\end{aligned}
$$

Upon introducing the expressions

$$
\begin{aligned}
d_{I}= & D F_{o}\left(\bar{U}_{o ; 0}+\Phi_{o}^{B}+\left(\Phi_{o}^{A}-\Phi_{o}^{B}\right)\right) \partial_{\xi}\left(\bar{U}_{o ; 0}+\Phi_{o}^{B}\right)-D F_{o}\left(\bar{U}_{o ; 0}+\Phi_{o}^{B}\right) \partial_{\xi}\left(\bar{U}_{o ; 0}+\Phi_{o}^{B}\right) \\
& -D^{2} F_{o}\left(\bar{U}_{o ; 0}+\Phi_{o}^{B}\right)\left[\Phi_{o}^{A}-\Phi_{o}^{B}, \partial_{\xi}\left(\bar{U}_{o ; 0}+\Phi_{o}^{B}\right)\right], \\
d_{I I}= & {\left[D F_{o}\left(\bar{U}_{o ; 0}+\Phi_{o}^{B}+\left(\Phi_{o}^{A}-\Phi_{o}^{B}\right)\right)-D F_{o}\left(\bar{U}_{o ; 0}+\Phi_{o}^{B}\right)\right] \partial_{\xi}\left(\Phi_{o}^{A}-\Phi_{o}^{B}\right), }
\end{aligned}
$$

we see that

$$
d_{1}+d_{2}=d_{I}+d_{I I} .
$$

Applying [17, Thm. 2.8.3] we obtain the bounds

$$
\begin{aligned}
\left|d_{I}\right| & \leq \frac{1}{2} K_{F}\left|\Phi_{o}^{A}-\Phi_{o}^{B}\right|^{2}\left[\left|\bar{U}_{o ; 0}^{\prime}\right|+\left|\partial_{\xi} \Phi_{o}^{B}\right|\right], \\
\left|d_{I I}\right| & \leq K_{F}\left|\Phi_{o}^{A}-\Phi_{o}^{B} \| \partial_{\xi}\left(\Phi_{o}^{A}-\Phi_{o}^{B}\right)\right| .
\end{aligned}
$$

In addition, the expressions

$$
\begin{aligned}
d_{I I I} & =\left[D F_{o}\left(\bar{U}_{o ; 0}+\Phi_{o}^{B}\right)-D F_{o}\left(\bar{U}_{o ; 0}\right)\right] \partial_{\xi}\left(\Phi_{o}^{A}-\Phi_{o}^{B}\right), \\
d_{I V} & =D^{2} F_{o}\left(\bar{U}_{o ; 0}+\Phi_{o}^{B}\right)\left[\bar{U}_{o ; 0}^{\prime}, \Phi_{o}^{A}-\Phi_{o}^{B}\right]-D^{2} F_{o}\left(\bar{U}_{o ; 0}\right)\left[\bar{U}_{o ; 0}^{\prime}, \Phi_{o}^{A}-\Phi_{o}^{B}\right], \\
d_{V} & =D^{2} F_{o}\left(\bar{U}_{o ; 0}+\Phi_{o}^{B}\right)\left[\partial_{\xi} \Phi_{o}^{B}, \Phi_{o}^{A}-\Phi_{o}^{B}\right]
\end{aligned}
$$

allow us to write

$$
d_{3}=d_{I I I}+d_{I V}+d_{V} .
$$

Applying [17, Thm. 2.8.3] we may estimate

$$
\begin{aligned}
\left|d_{I I I}\right| & \leq K_{F}\left|\Phi_{o}^{B} \| \partial_{\xi}\left(\Phi_{o}^{A}-\Phi_{o}^{B}\right)\right|, \\
\left|d_{I V}\right| & \leq K_{F}\left|\Phi_{o}^{B} \| \Phi_{o}^{A}-\Phi_{o}^{B}\right|, \\
\left|d_{V}\right| & \leq K_{F}\left|\partial_{\xi} \Phi_{o}^{B}\right|\left|\Phi_{o}^{A}-\Phi_{o}^{B}\right| .
\end{aligned}
$$

These bounds can all be absorbed into (5.28). The estimate for $\mathcal{N}_{e}$ follows similarly.

Copyright (๑) by SIAM. Unauthorized reproduction of this article is prohibited. 
With the above pointwise bounds in hand, we are ready to estimate the nonlinearities in the appropriate scaled function spaces. To this end, we introduce the notation

$$
\mathcal{N}(\Phi)=\left(\mathcal{N}_{o}\left(\Phi_{o}\right), \mathcal{N}_{e}\left(\Phi_{e}\right)\right)
$$

for any $\Phi=\left(\Phi_{o}, \Phi_{e}\right) \in \mathbf{H}^{1}$.

LEmma 5.6. Assume that (HN1), (HN2), (HW1), and (HW2) are satisfied. There exists a constant $K_{\mathcal{N}}>0$ so that for each $0<\eta \leq \eta_{*}$, each $\varepsilon>0$, and each triplet $\left(\Phi, \Phi^{A}, \Phi^{B}\right) \in \mathbf{X}_{\eta ; \varepsilon}^{3}$ we have the bounds

$$
\begin{aligned}
\left\|\mathcal{M}_{\varepsilon}^{1} \mathcal{N}(\Phi)\right\|_{\mathbf{L}^{2}} & \leq K_{\mathcal{N}} \eta^{2} \\
\left\|\mathcal{M}_{\varepsilon}^{1,2} \partial_{\xi} \mathcal{N}(\Phi)\right\|_{\mathbf{L}^{2}} & \leq K_{\mathcal{N}} \eta^{2} \\
\left\|\mathcal{M}_{\varepsilon}^{1}\left(\mathcal{N}\left(\Phi^{A}\right)-\mathcal{N}\left(\Phi^{B}\right)\right)\right\|_{\mathbf{L}^{2}} & \leq K_{\mathcal{N}} \eta\left\|\Phi^{A}-\Phi^{B}\right\|_{\mathbf{L}^{2}} \\
\left\|\mathcal{M}_{\varepsilon}^{1,2} \partial_{\xi}\left(\mathcal{N}\left(\Phi^{A}\right)-\mathcal{N}\left(\Phi^{B}\right)\right)\right\|_{\mathbf{L}^{2}} & \leq K_{\mathcal{N}} \eta\left(\left\|\Phi^{A}-\Phi^{B}\right\|_{\mathbf{L}^{2}}+\left\|\partial_{\xi}\left(\Phi^{A}-\Phi^{B}\right)\right\|_{\mathbf{L}^{2}}\right) .
\end{aligned}
$$

Proof. All bounds follow immediately from Lemmas 5.2 and 5.5 upon using the Sobolev estimate $\|\phi\|_{\infty} \leq C_{1}^{\prime}\|\phi\|_{H^{1}}$ to write

$$
\begin{array}{rlrl}
\left\|\Phi_{o}\right\|_{\infty} & \leq C_{1}^{\prime} \eta, & \left\|\partial_{\xi} \Phi_{o}\right\|_{\infty} & \leq C_{1}^{\prime} \frac{\eta}{\varepsilon} \\
\left\|\Phi_{e}\right\|_{\infty} & \leq C_{1}^{\prime} \eta, & \left\|\partial_{\xi} \Phi_{e}\right\|_{\infty} \leq C_{1}^{\prime} \eta
\end{array}
$$

with identical bounds for $\Phi^{A}$ and $\Phi^{B}$.

Lemma 5.7. Assume that (HN1), (HN2), (HW1), and (HW2) are satisfied. Then there exists a constant $K_{\mathcal{E}}>0$ so that for each $\varepsilon>0$ we have the bound

$$
\left\|\mathcal{M}_{\varepsilon}^{1} \mathcal{E}_{0}\right\|_{\mathbf{L}^{2}}+\left\|\mathcal{M}_{\varepsilon}^{1,2} \partial_{\xi} \mathcal{E}_{0}\right\|_{\mathbf{L}^{2}} \leq \varepsilon K_{\mathcal{E}}
$$

Proof. The structure of the matrix $J$ allows us to bound

$$
\left\|\mathcal{M}_{\varepsilon}^{1} \mathcal{E}_{0}\right\|_{\mathbf{L}^{2}} \leq \varepsilon\left\|\mathcal{E}_{0}\right\|_{\mathbf{L}^{2}}, \quad\left\|\mathcal{M}_{\varepsilon}^{1,2} \partial_{\xi} \mathcal{E}_{0}\right\|_{\mathbf{L}^{2}} \leq \varepsilon\left\|\partial_{\xi} \mathcal{E}_{0}\right\|_{\mathbf{L}^{2}}
$$

The result hence follows from the inclusions

$$
\bar{U}_{o ; 0}^{\prime} \in \mathbf{H}_{o}^{1}, \quad F_{o}\left(\bar{U}_{o ; 0}\right) \in \mathbf{H}_{o}^{1} .
$$

The first of these can be obtained by differentiating (2.18) and (2.20). The second inclusion follows from the fact that $\bar{U}_{o ; 0}$ converges exponentially fast to its limiting values, which are zeroes of $F_{o}$.

LEMMA 5.8. Assume that (HN1), (HN2), (HW1), (HW2), and (HS1) are satisfied. Then there exists a constant $K_{c}>0$ in such a way that for each $0<\eta \leq \eta_{*}$, each $\varepsilon>0$, each $\delta>0$, and each triplet $\left(\Phi, \Phi^{A}, \Phi^{B}\right) \in \mathbf{X}_{\eta ; \varepsilon}^{3}$ we have the bounds

$$
\begin{aligned}
\left|c_{\delta}\left(\Phi_{e}\right)-c_{0}\right| & \leq K_{c}\left[\delta \eta+\eta^{2}\right], \\
\left|c_{\delta}\left(\Phi_{e}^{A}\right)-c_{\delta}\left(\Phi_{e}^{B}\right)\right| & \leq K_{c}(\delta+\eta)\left\|\Phi^{A}-\Phi^{B}\right\|_{\mathbf{L}^{2}} .
\end{aligned}
$$

Proof. Since we only need to use regular $L^{2}$-norms for these estimates, the proof of $[43$, Lem. 4.4] also applies here.

Copyright (C) by SIAM. Unauthorized reproduction of this article is prohibited. 
Lemma 5.9. Assume that (HN1), (HN2), (HW1), (HW2), and (HS1) are satisfied. Then there exists a constant $K_{\mathcal{R}}>0$ in such a way that for each $0<\eta \leq \eta_{*}$, each $0<\varepsilon<1$, each $\delta>0$, and each triplet $\left(\Phi, \Phi^{A}, \Phi^{B}\right) \in \mathbf{X}_{\eta ; \varepsilon}^{3}$ we have the bound

$$
\left\|\mathcal{M}_{\varepsilon}^{1} \mathcal{R}\left(c_{\delta}\left(\Phi_{e}\right), \Phi\right)\right\|_{\mathbf{L}^{2}}+\left\|\mathcal{M}_{\varepsilon}^{1,2} \partial_{\xi} \mathcal{R}\left(c_{\delta}\left(\Phi_{e}\right), \Phi\right)\right\|_{\mathbf{L}^{2}} \leq K_{\mathcal{R}}\left[\delta \eta+\eta^{2}\right] .
$$

Writing

$$
\Delta_{A B} \mathcal{R}:=\mathcal{R}\left(c_{\delta}\left(\Phi_{e}^{A}\right), \Phi^{A}\right)-\mathcal{R}\left(c_{\delta}\left(\Phi_{e}^{B}\right), \Phi^{B}\right),
$$

we also have the bound

$$
\begin{aligned}
\left\|\mathcal{M}_{\varepsilon}^{1} \Delta_{A B} \mathcal{R}\right\|_{\mathbf{L}^{2}}+\left\|\mathcal{M}_{\varepsilon}^{1,2} \partial_{\xi} \Delta_{A B} \mathcal{R}\right\|_{\mathbf{L}^{2}} \leq & K_{\mathcal{R}}(\delta+\eta)\left\|\Phi^{A}-\Phi^{B}\right\|_{\mathbf{L}^{2}} \\
& +\eta K_{\mathcal{R}}(\eta+\delta)\left\|\partial_{\xi}\left(\Phi^{A}-\Phi^{B}\right)\right\|_{\mathbf{L}^{2}} \\
& +\eta K_{\mathcal{R}}(\eta+\delta)\left\|\mathcal{M}_{\varepsilon}^{1,2} \partial_{\xi}^{2}\left(\Phi^{A}-\Phi^{B}\right)\right\|_{\mathbf{L}^{2}} .
\end{aligned}
$$

Proof. Using Lemma 5.8 we immediately obtain the bound

$$
\begin{aligned}
\left\|\mathcal{M}_{\varepsilon}^{1} \mathcal{R}\left(c_{\delta}\left(\Phi_{e}\right), \Phi\right)\right\|_{\mathbf{L}^{2}} & \leq K_{c}\left[\delta \eta+\eta^{2}\right]\left(\left\|\mathcal{M}_{\varepsilon}^{1} \partial_{\xi} \Phi\right\|_{\mathbf{L}^{2}}+\left\|\mathcal{M}_{\varepsilon}^{1} \bar{U}_{0}^{\prime}\right\|_{\mathbf{L}^{2}}\right) \\
& \leq K_{c}\left[\delta \eta+\eta^{2}\right]\left(\eta+\left\|\bar{U}_{0}^{\prime}\right\|_{\mathbf{L}^{2}}\right)
\end{aligned}
$$

together with

$$
\begin{aligned}
\left\|\mathcal{M}_{\varepsilon}^{1,2} \partial_{\xi} \mathcal{R}\left(c_{\delta}\left(\Phi_{e}\right), \Phi\right)\right\|_{\mathbf{L}^{2}} & \leq K_{c}\left[\delta \eta+\eta^{2}\right]\left(\left\|\mathcal{M}_{\varepsilon}^{1,2} \partial_{\xi}^{2} \Phi\right\|_{\mathbf{L}^{2}}+\left\|\mathcal{M}_{\varepsilon}^{1,2} \bar{U}_{0}^{\prime \prime}\right\|_{\mathbf{L}^{2}}\right) \\
& \leq K_{c}\left[\delta \eta+\eta^{2}\right]\left(\eta+\left\|\bar{U}_{0}^{\prime \prime}\right\|_{\mathbf{L}^{2}}\right) .
\end{aligned}
$$

In addition, we may compute

$$
\begin{array}{r}
\Delta_{A B} \mathcal{R}=\left(c_{\delta}\left(\Phi_{e}^{B}\right)-c_{\delta}\left(\Phi_{e}^{A}\right)\right) \partial_{\xi}\left(\bar{U}_{0}+\Phi^{A}\right) \\
+\left(c_{0}-c_{\delta}\left(\Phi_{e}^{B}\right)\right) \partial_{\xi}\left(\Phi^{A}-\Phi^{B}\right)
\end{array}
$$

which allows us to estimate

$$
\begin{gathered}
\left\|\mathcal{M}_{\varepsilon}^{1} \Delta_{A B} \mathcal{R}\right\|_{\mathbf{L}^{2}} \leq K_{c}(\delta+\eta)\left\|\Phi^{A}-\Phi^{B}\right\|_{\mathbf{L}^{2}}\left(\left\|\mathcal{M}_{\varepsilon}^{1} \bar{U}_{0}^{\prime}\right\|_{\mathbf{L}^{2}}+\left\|\mathcal{M}_{\varepsilon}^{1} \partial_{\xi} \Phi^{A}\right\|_{\mathbf{L}^{2}}\right) \\
+K_{c}\left[\delta \eta+\eta^{2}\right]\left\|\mathcal{M}_{\varepsilon}^{1} \partial_{\xi}\left(\Phi^{A}-\Phi^{B}\right)\right\|_{\mathbf{L}^{2}} \\
\leq K_{c}(\delta+\eta)\left\|\Phi^{A}-\Phi^{B}\right\|_{\mathbf{L}^{2}}\left(\left\|\bar{U}_{0}^{\prime}\right\|_{\mathbf{L}^{2}}+\eta\right) \\
+K_{c}\left[\delta \eta+\eta^{2}\right]\left\|\partial_{\xi}\left(\Phi^{A}-\Phi^{B}\right)\right\|_{\mathbf{L}^{2}}
\end{gathered}
$$

together with

$$
\begin{gathered}
\left\|\mathcal{M}_{\varepsilon}^{1,2} \partial_{\xi} \Delta_{A B} \mathcal{R}\right\|_{\mathbf{L}^{2}} \leq K_{c}(\delta+\eta)\left\|\Phi^{A}-\Phi^{B}\right\|_{\mathbf{L}^{2}}\left(\left\|\mathcal{M}_{\varepsilon}^{1,2} \bar{U}_{0}^{\prime \prime}\right\|_{\mathbf{L}^{2}}+\left\|\mathcal{M}_{\varepsilon}^{1,2} \partial_{\xi} \Phi^{A}\right\|_{\mathbf{L}^{2}}\right) \\
+K_{c}\left[\delta \eta+\eta^{2}\right]\left\|\mathcal{M}_{\varepsilon}^{1,2} \partial_{\xi}^{2}\left(\Phi^{A}-\Phi^{B}\right)\right\|_{\mathbf{L}^{2}} \\
\leq K_{c}(\delta+\eta)\left\|\Phi^{A}-\Phi^{B}\right\|_{\mathbf{L}^{2}}\left(\left\|\bar{U}_{0}^{\prime \prime}\right\|_{\mathbf{L}^{2}}+\eta\right) \\
+K_{c}\left[\delta \eta+\eta^{2}\right]\left\|\mathcal{M}_{\varepsilon}^{1,2} \partial_{\xi}\left(\Phi^{A}-\Phi^{B}\right)\right\|_{\mathbf{L}^{2}}
\end{gathered}
$$

These terms can all be absorbed into (5.46).

Copyright (C) by SIAM. Unauthorized reproduction of this article is prohibited. 
Proof of Theorem 2.1. Using Lemmas 5.6, 5.7, and 5.9, together with the decomposition (5.5) and the estimates (5.14)-(5.15), we find that there exists a constant $K_{T}>0$ for which the bounds

$$
\begin{aligned}
\left\|T_{\varepsilon, \delta}(\Phi)\right\|_{\mathbf{X}_{\varepsilon}} & \leq K_{T}\left[\delta \eta+\eta^{2}+\varepsilon\right] \\
\left\|T_{\varepsilon, \delta}\left(\Phi^{A}\right)-T_{\varepsilon, \delta}\left(\Phi^{B}\right)\right\|_{\mathbf{X}_{\varepsilon}} & \leq K_{T}[\delta+\eta]\left\|\Phi^{A}-\Phi^{B}\right\|_{\mathbf{X}_{\varepsilon}}
\end{aligned}
$$

hold for any $\eta \leq \eta_{*}$, any $0<\varepsilon<\varepsilon_{0}(\delta)$, and any triplet $\left(\Phi, \Phi^{A}, \Phi^{B}\right) \in \mathbf{X}_{\eta ; \varepsilon}^{3}$. As such, we fix

$$
\delta=\frac{1}{3 K_{T}}, \quad \eta=\min \left\{\eta_{*}, \frac{1}{3 K_{T}}\right\} .
$$

Finally, we select a small positive $\varepsilon_{*}$ such that $\varepsilon_{*} \leq \varepsilon_{0}(\delta)$ and $\varepsilon_{*} \leq \frac{1}{3 K_{T}} \eta$. We conclude that for each $0<\varepsilon \leq \varepsilon_{*}, T_{\varepsilon, \delta}$ maps $\mathbf{X}_{\eta ; \varepsilon}$ into itself and is a contraction. This completes the proof.

6. Stability of traveling waves. Introducing the family

$$
\left(\tilde{U}_{\varepsilon}, \tilde{c}_{\varepsilon}\right)=\left(\bar{U}_{\varepsilon}, c_{\varepsilon}\right)
$$

which satisfies (hFam) on account of Theorem 2.1, we see that the theory developed in section 4 applies to the operators

$$
\overline{\mathcal{L}}_{\varepsilon, \lambda}: \mathbf{H}^{1} \rightarrow \mathbf{L}^{2}
$$

that act as

$$
\overline{\mathcal{L}}_{\varepsilon, \lambda}=c_{\varepsilon} \frac{d}{d \xi}-\mathcal{M}_{1 / \varepsilon^{2}}^{1} J_{\text {mix }}-D F\left(\bar{U}_{\varepsilon}\right)+\lambda .
$$

We emphasize that these operators are associated to the linearization of the traveling wave system $(2.14)$ around the wave solutions $\left(\bar{U}_{\varepsilon}, c_{\varepsilon}\right)$. For convenience, we also introduce the shorthand

$$
\overline{\mathcal{L}}_{\varepsilon}=\overline{\mathcal{L}}_{\varepsilon, 0}=c_{\varepsilon} \frac{d}{d \xi}-\mathcal{M}_{1 / \varepsilon^{2}}^{1} J_{\text {mix }}-D F\left(\bar{U}_{\varepsilon}\right) .
$$

We remark that the spectrum of $\overline{\mathcal{L}}_{\varepsilon}$ is $2 \pi i c_{\varepsilon}$-periodic on account of the identity

$$
\left(\overline{\mathcal{L}}_{\varepsilon}+\lambda\right) e^{2 \pi i \cdot}=e^{2 \pi i \cdot}\left(\overline{\mathcal{L}}_{\varepsilon}+\lambda+2 \pi i c_{\varepsilon}\right) .
$$

As a final preparation, we note that there exists a constant $\bar{K}_{F}>0$ for which the bound

$$
\left\|D F_{o}\left(\bar{U}_{o ; \varepsilon}\right)\right\|_{\infty}+\left\|D^{2} F_{o}\left(\bar{U}_{o ; \varepsilon}\right)\right\|_{\infty}+\left\|D F_{e}\left(\bar{U}_{e ; \varepsilon}\right)\right\|_{\infty}+\left\|D^{2} F_{e}\left(\bar{U}_{e ; \varepsilon}\right)\right\|_{\infty} \leq \bar{K}_{F}
$$

holds for all $0<\varepsilon<\varepsilon_{*}$.

Our main task here is to reverse the parameter dependency used in section 4 . In particular, for a fixed small value of $\varepsilon>0$ we study the behavior of the map $\lambda \mapsto \overline{\mathcal{L}}_{\varepsilon, \lambda}$. This allows us to obtain the main result of this section, which lifts the spectral stability assumptions (HS1) and (HS2) to the full system (2.14).

Proposition 6.1. Assume that (HN1), (HN2), (HW1), (HW2), (HS1), and (HS2) are satisfied. Then there exists a constant $\varepsilon_{* *}>0$ so that the following properties hold for all $0<\varepsilon<\varepsilon_{* *}$. 
(i) We have

$$
\operatorname{Ker}\left(\overline{\mathcal{L}}_{\varepsilon}\right)=\operatorname{span}\left(\bar{U}_{\varepsilon}^{\prime}\right)
$$

together with $\bar{U}_{\varepsilon}^{\prime} \notin$ Range $\left(\overline{\mathcal{L}}_{\varepsilon, 0}\right)$.

(ii) For each $\lambda \in \mathbb{C} \backslash 2 \pi i c_{\varepsilon} \mathbb{Z}$ with $\operatorname{Re} \lambda \geq-\lambda_{*}$, the operator $\overline{\mathcal{L}}_{\varepsilon, \lambda}$ is invertible.

These spectral stability properties can be turned into a nonlinear stability result by applying the theory developed in [30]. The main idea is to consider a temporal Green's function for the LDE (2.1) and spatial Green's functions for the traveling wave equation (2.14). These Green's functions can be related to each other using an integral representation. Our detailed knowledge of the spectrum of the operator $\overline{\mathcal{L}}_{\varepsilon}$ allows us to shift the integration path and split the temporal Green's function for the linearization of (2.1) around the wave $\bar{U}_{\varepsilon}$ into two components. The first corresponds to the neutral part of the flow along the eigenfunction $\bar{U}_{\varepsilon}^{\prime}$, while the second encodes the exponentially decaying stable part of the flow. The full orbital neighborhood of the traveling wave $\bar{U}_{\varepsilon}$ can now be spanned by the family of stable manifolds for the shifted waves $\bar{U}_{\varepsilon}(\cdot+\vartheta)$, which all have codimension one. In particular, every initial condition in this neighborhood converges exponentially to a shifted version of $\bar{U}_{\varepsilon}$.

Proof of Theorem 2.2. For $j \in \mathbb{Z}$ we introduce the new variables

$$
\left(u_{j ; o}, w_{j ; o}, u_{j ; e}, w_{j ; e}\right)=\left(u_{2 j+1}, w_{2 j+1}, u_{2 j}, w_{2 j}\right),
$$

which allows us to reformulate the 2-periodic system (2.1) as the equivalent $2(n+k)$ component system

$$
\begin{aligned}
& \dot{u}_{j ; o}(t)=\frac{1}{\varepsilon^{2}} \mathcal{D}\left[u_{j+1 ; e}(t)+u_{j ; e}(t)-2 u_{j ; o}(t)\right]+f_{o}\left(u_{j ; o}(t), w_{j ; o}(t)\right), \\
& \dot{u}_{j ; o}(t)=g_{o}\left(u_{j ; o}(t), w_{j ; o}(t)\right), \\
& \dot{u}_{j ; e}(t)=\mathcal{D}\left[u_{j ; o}(t)+u_{j-1 ; o}(t)-2 u_{j ; e}(t)\right]+f_{e}\left(u_{j ; e}(t), w_{j ; e}(t)\right), \\
& \dot{w}_{j ; e}(t)=g_{e}\left(u_{j ; e}(t), w_{j ; e}(t)\right),
\end{aligned}
$$

which is spatially homogeneous.

On account of Theorem 2.1 and Proposition 6.1, it is clear that (6.9) satisfies the conditions (HV), (HS1)-(HS3) from [30]. An application of [30, Prop. 2.1] immediately yields the desired result.

6.1. The operator $\overline{\mathcal{L}}_{\varepsilon}$. Observe first that $\overline{\mathcal{L}}_{\varepsilon}$ is a Fredholm operator with index zero on account of Lemma 4.6. Our goal in this subsection is to establish the characterization of the kernel and range of this operator given in item (i) of Proposition 6.1. We note that this statement implies that the zero eigenvalue of $\overline{\mathcal{L}}_{\mathcal{E}}$ is simple.

At times, our discussion closely follows the lines of [44, sects. 4-5]. The novel ingredient here, however, is that we do not need to modify the spectral convergence argument from section 4 to ensure that it also applies to the adjoint operator. Indeed, we show that all the essential properties can be obtained from the following quasiinverse for $\overline{\mathcal{L}}_{\varepsilon}$, which can be constructed by mimicking the approach of [32, Prop. $3.2]$.

Lemma 6.2. Assume that (HN1), (HN2), (HW1), (HW2), (HS1), and (HS2) are satisfied and pick a sufficiently small constant $\varepsilon_{* *}>0$. Then for every $0<\varepsilon<\varepsilon_{* *}$ there exist linear maps

$$
\begin{gathered}
\bar{\gamma}_{\varepsilon}: \mathbf{L}^{2} \rightarrow \mathbb{R}, \\
\overline{\mathcal{L}}_{\varepsilon}^{\text {qinv }}: \mathbf{L}^{2} \rightarrow \mathbf{H}^{1},
\end{gathered}
$$


so that for all $\Theta \in \mathbf{L}^{2}$ the pair

$$
(\gamma, \Psi)=\left(\bar{\gamma}_{\varepsilon} \Theta, \overline{\mathcal{L}}_{\varepsilon}^{\text {qinv }} \Theta\right)
$$

is the unique solution to the problem

$$
\overline{\mathcal{L}}_{\varepsilon} \Psi=\Theta+\gamma \bar{U}_{0}^{\prime}
$$

that satisfies the normalization condition

$$
\left\langle\left(0, \Phi_{e ; 0}^{\mathrm{adj}}\right), \Psi\right\rangle_{\mathbf{L}^{2}}=0 .
$$

In addition, there exists $C>0$ such that for all $0<\varepsilon<\varepsilon_{* *}$ and all $\Theta \in \mathbf{L}^{2}$ we have the bound

$$
\left|\bar{\gamma}_{\varepsilon} \Theta\right|+\left\|\mathcal{M}_{\varepsilon}^{1}\left(\overline{\mathcal{L}}_{\varepsilon}^{\text {qinv }} \Theta\right)^{\prime}\right\|_{\mathbf{L}^{2}}+\left\|\overline{\mathcal{L}}_{\varepsilon}^{\text {qinv }} \Theta\right\|_{\mathbf{L}^{2}} \leq C\left\|\mathcal{M}_{\varepsilon}^{1} \Theta\right\|_{\mathbf{L}^{2}}
$$

Proof. The proof of [44, Lem. 4.9] remains valid in this setting.

We can now concentrate on the kernel of $\overline{\mathcal{L}}_{\varepsilon}$. The quasi-inverse constructed above allows us to develop a Liapunov-Schmidt argument to exclude kernel elements other than $\bar{U}_{\varepsilon}^{\prime}$.

Lemma 6.3. Assume that (HN1), (HN2), (HW1), (HW2), (HS1), and (HS2) are satisfied. Then for all sufficiently small $\varepsilon>0$ we have

$$
\operatorname{ker}\left(\overline{\mathcal{L}}_{\varepsilon}\right)=\operatorname{span}\left\{\bar{U}_{\varepsilon}^{\prime}\right\} .
$$

Proof. This result can be obtained by following the procedure used in the proof of [44, Lems. 4.10-4.11].

We now set out to show that the eigenfunction $\bar{U}_{\varepsilon}^{\prime}$ is in fact simple. As a technical preparation, we obtain a lower bound on $\bar{\gamma}_{\varepsilon}\left(\bar{U}_{\varepsilon}^{\prime}\right)$, which will help us to exploit the quasi-inverse constructed in Lemma 6.2.

Lemma 6.4. Assume that (HN1), (HN2), (HW1), (HW2), (HS1), and (HS2) are satisfied. Then there exists a constant $\gamma_{*}>0$ so that the inequality

$$
\left|\bar{\gamma}_{\varepsilon} \bar{U}_{\varepsilon}^{\prime}\right| \geq \gamma_{*}
$$

holds for all sufficiently small $\varepsilon>0$.

Proof. We note first that the limit $\bar{U}_{\varepsilon}^{\prime} \rightarrow \bar{U}_{0}^{\prime}$ in $\mathbf{L}^{2}$ and the inequality $\left\langle\bar{U}_{e ; 0}^{\prime}, \Phi_{e ; 0}^{\text {adj }}\right\rangle_{\mathbf{L}_{e}^{2}}$ $\neq 0$ imply that there exists a constant $\nu_{*}>0$ so that

$$
\left|\left\langle\bar{U}_{\varepsilon}^{\prime},\left(0, \Phi_{e ; 0}^{\mathrm{adj}}\right)\right\rangle_{\mathbf{L}^{2}}\right| \geq \nu_{*}
$$

for all small $\varepsilon>0$.

We now introduce the function

$$
\Psi_{\varepsilon}=\overline{\mathcal{L}}_{\varepsilon}^{\mathrm{qinv}} \bar{U}_{\varepsilon}^{\prime} .
$$

The uniform bound (6.14) shows that we may assume an a priori bound of the form

$$
\left\|\Psi_{\varepsilon}\right\|_{\mathbf{L}^{2}} \leq C_{1}^{\prime}
$$

for some $C_{1}^{\prime}>0$.

Copyright (c) by SIAM. Unauthorized reproduction of this article is prohibited. 
For any sufficiently small $\delta>0$ and $0<\varepsilon<\varepsilon_{0}(\delta)$, the explicit form of $\bar{\gamma}_{\varepsilon}$ given in $[44$, eq. (4.47)] implies that

$$
\begin{aligned}
\bar{\gamma}_{\varepsilon} \bar{U}_{\varepsilon}^{\prime} & =\frac{\left\langle\left(0, \Phi_{e j 0}^{\mathrm{adj}}\right),\left(\overline{\mathcal{L}}_{\varepsilon}+\delta\right)^{-1}\left(\bar{U}_{\varepsilon}^{\prime}+\delta \Psi_{\varepsilon}\right)\right\rangle_{\mathbf{L}^{2}}}{\left\langle\left(0, \Phi_{e j 0}^{\mathrm{adj}}\right),\left(\overline{\mathcal{L}}_{\varepsilon}+\delta\right)^{-1} \bar{U}_{0}^{\prime}\right\rangle_{\mathbf{L}^{2}}} \\
& =\frac{\left\langle\left(0, \Phi_{e ; 0}^{\mathrm{adj}}\right), \delta^{-1} \bar{U}_{\varepsilon}^{\prime}+\left(\overline{\mathcal{L}}_{\varepsilon}+\delta\right)^{-1} \delta \Psi_{\varepsilon}\right\rangle_{\mathbf{L}^{2}}}{\left\langle\left(0, \Phi_{e ; 0}^{\mathrm{adj}}\right),\left(\overline{\mathcal{L}}_{\varepsilon}+\delta\right)^{-1} \bar{U}_{0}^{\prime}\right\rangle_{\mathbf{L}^{2}}} .
\end{aligned}
$$

Since $\left(\overline{\mathcal{L}}_{\varepsilon}+\delta\right)^{-1} \delta \Psi_{\varepsilon}$ is uniformly bounded in $\mathbf{L}^{2}$ for all sufficiently small $\delta>0$ and $0<\varepsilon<\varepsilon_{0}(\delta)$ on account of Corollary 4.4 and (6.19), we can use the lower bound (6.17) to assume that $\delta>0$ is small enough to have

$$
\left|\left\langle\left(0, \Phi_{e ; 0}^{\mathrm{adj}}\right), \delta^{-1} \bar{U}_{\varepsilon}^{\prime}+\left(\overline{\mathcal{L}}_{\varepsilon}+\delta\right)^{-1} \delta \Psi_{\varepsilon}\right\rangle_{\mathbf{L}^{2}}\right| \geq C_{2}^{\prime} \delta^{-1}
$$

for all such $(\varepsilon, \delta)$. Moreover, the uniform bound in Corollary 4.4 also yields the upper bound

$$
\left|\left\langle\left(0, \Phi_{e ; 0}^{\mathrm{adj}}\right),\left(\overline{\mathcal{L}}_{\varepsilon}+\delta\right)^{-1} \bar{U}_{0}^{\prime}\right\rangle_{\mathbf{L}^{2}}\right| \leq C_{3}^{\prime}\left(1+\delta^{-1}\right)
$$

for all such $(\varepsilon, \delta)$. This gives us the lower bound

$$
\left|\bar{\gamma}_{\varepsilon} \bar{U}_{\varepsilon}^{\prime}\right| \geq \frac{C_{2}^{\prime}}{C_{3}^{\prime}} \frac{\delta^{-1}}{1+\delta^{-1}} \geq \gamma_{*}
$$

for some $\gamma_{*}>0$ that can be chosen independently of $\delta>0$.

Lemma 6.5. Assume that (HN1), (HN2), (HW1), (HW2), (HS1), and (HS2) are satisfied. Then for all sufficiently small $\varepsilon>0$ we have $\bar{U}_{\varepsilon}^{\prime} \notin \operatorname{Range}\left(\overline{\mathcal{L}}_{\varepsilon}\right)$.

Proof. Arguing by contradiction, let us suppose that there exists $\Psi_{\varepsilon} \in \mathbf{H}^{1}$ for which the identity

$$
\overline{\mathcal{L}}_{\varepsilon} \Psi_{\varepsilon}=\bar{U}_{\varepsilon}^{\prime}
$$

holds. The observation above allows us to add an appropriate multiple of $\bar{U}_{\varepsilon}^{\prime}$ to $\Psi_{\varepsilon}$ to ensure that $\left\langle\Psi_{\varepsilon},\left(0, \Phi_{e ; 0}^{\mathrm{adj}}\right)\right\rangle_{\mathbf{L}^{2}}=0$. In particular, Lemma 6.2 implies that

$$
\bar{\gamma}_{\varepsilon} \bar{U}_{\varepsilon}^{\prime}=0, \quad \overline{\mathcal{L}}_{\varepsilon}^{\text {qinv }} \bar{U}_{\varepsilon}^{\prime}=\Psi_{\varepsilon},
$$

which immediately contradicts Lemma 6.4.

6.2. Spectral stability. Here we set out to establish the statements in Proposition 6.1 for $\lambda \notin 2 \pi i c_{\varepsilon} \mathbb{Z}$. In contrast to the setting in [44], the period $2 \pi i c_{\varepsilon}$ can be uniformly bounded for $\varepsilon \downarrow 0$. In particular, we will only consider values of $\varepsilon>0$ that are sufficiently small to ensure that

$$
\frac{3}{4} c_{0}<c_{\varepsilon}<\frac{3}{2} c_{0}
$$

holds. Recalling the constant $\lambda_{0}$ introduced in Proposition 4.2, this allows us to restrict our spectral analysis to the set

$$
\mathcal{R}:=\left\{\lambda \in \mathbb{C}: \operatorname{Re} \lambda \geq-\lambda_{0},|\operatorname{Im} \lambda| \leq \frac{3}{2} \pi c_{0}\right\} \backslash\{0\} .
$$

Copyright $@$ ㅇ by SIAM. Unauthorized reproduction of this article is prohibited. 


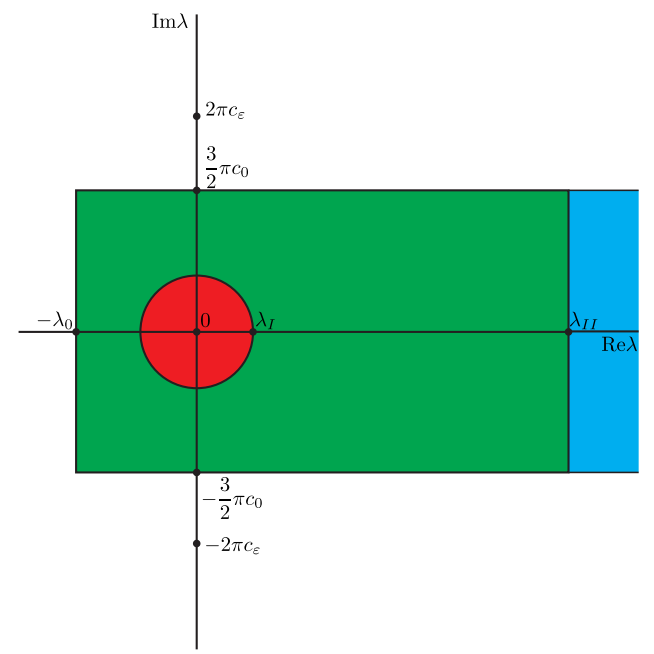

FIG. 6.1. Illustration of the decomposition of the spectrum into $\varepsilon$-independent regions.

On account of Lemma 4.6, the operators $\overline{\mathcal{L}}_{\varepsilon, \lambda}$ are all Fredholm with index 0 on this set. We hence only need to establish their injectivity.

It turns out to be convenient to partition this strip into three $\varepsilon$-independent parts, which we illustrate in Figure 6.1. The first part (red) contains values of $\lambda$ that are close to 0 , which can be analyzed using the theory developed in subsection 6.1 . The second part (blue) contains all values of $\lambda$ for which $\operatorname{Re} \lambda$ is sufficiently large. Such values can be excluded from the spectrum by straightforward norm estimates. The remaining part (green) is compact, which allows us to appeal to Proposition 4.2.

Lemma 6.6. Assume that (HN1), (HN2), (HW1), (HW2), (HS1), and (HS2) are satisfied. There exists constants $\lambda_{I}>0$ and $\varepsilon_{I}>0$ so that the operator $\overline{\mathcal{L}}_{\varepsilon, \lambda}: \mathbf{H}^{1} \rightarrow$ $\mathbf{L}^{2}$ is injective for all $\lambda \in \mathbb{C}$ with $0<|\lambda|<\lambda_{I}$ and $0<\varepsilon<\varepsilon_{I}$.

Proof. We argue by contradiction. Pick a small $\lambda_{I}>0$ and $0<\varepsilon<\varepsilon_{* *}$ and assume that there exists $\Psi \in \mathbf{H}^{1}$ and $0<|\lambda|<\lambda_{I}$ with $\Psi \neq 0$ and

$$
\overline{\mathcal{L}}_{\varepsilon} \Psi=\lambda \Psi .
$$

Aiming to exploit the quasi-inverse in Lemma 6.2, we use (6.17) to decompose $\Psi$ as

$$
\Psi=\kappa \bar{U}_{\varepsilon}^{\prime}+\Psi^{\perp}
$$

for some $\kappa \in \mathbb{R}$ and $\Psi^{\perp} \in \mathbf{H}^{1}$ that satisfies the normalization condition

$$
\left\langle\left(0, \Phi_{e ; 0}^{\mathrm{adj}}\right), \Psi^{\perp}\right\rangle_{\mathbf{L}^{2}}=0 .
$$

In view of Lemma 6.2, the identity (6.28) implies that

$$
\bar{\gamma}_{\varepsilon}\left[\kappa \lambda \bar{U}_{\varepsilon}^{\prime}+\lambda \Psi^{\perp}\right]=0, \quad \overline{\mathcal{L}}_{\varepsilon}^{\text {qinv }}\left[\kappa \lambda \bar{U}_{\varepsilon}^{\prime}+\lambda \Psi^{\perp}\right]=\Psi^{\perp} .
$$

On account of the uniform bound (6.14), we can assume that $\lambda_{I}$ is small enough to have

$$
\lambda_{I}\left\|\overline{\mathcal{L}}_{\varepsilon}^{\text {qinv }}\right\|_{\mathcal{B}\left(\mathbf{L}^{2} ; \mathbf{L}^{2}\right)}<\frac{1}{2} .
$$


Since $|\lambda|<\lambda_{I}$, this means that we can rewrite (6.31) to obtain

$$
\Psi^{\perp}=\left[I-\lambda \overline{\mathcal{L}}_{\varepsilon}^{\text {qinv }}\right]^{-1} \overline{\mathcal{L}}_{\varepsilon}^{\text {qinv }}\left[\kappa \lambda \bar{U}_{\varepsilon}^{\prime}\right] .
$$

In particular, the first identity in (6.31) allows us to write

$$
\begin{aligned}
0 & =\bar{\gamma}_{\varepsilon}\left[\kappa \lambda \bar{U}_{\varepsilon}^{\prime}+\lambda\left[I-\lambda \overline{\mathcal{L}}_{\varepsilon}^{\mathrm{qinv}}\right]^{-1} \overline{\mathcal{L}}_{\varepsilon}^{\mathrm{qinv}}\left[\kappa \lambda \bar{U}_{\varepsilon}^{\prime}\right]\right] \\
& =\kappa \lambda \bar{\gamma}_{\varepsilon}\left[\bar{U}_{\varepsilon}^{\prime}+\lambda\left[I-\lambda \overline{\mathcal{L}}_{\varepsilon}^{\mathrm{qinv}}\right]^{-1} \overline{\mathcal{L}}_{\varepsilon}^{\mathrm{qinv}}\left[\bar{U}_{\varepsilon}^{\prime}\right]\right] .
\end{aligned}
$$

We note that the restriction (6.32) ensures that the second identity in (6.31) has no nonzero solutions $\Psi^{\perp}$ for $\kappa=0$. In particular, (6.34) implies that we must have

$$
\bar{\gamma}_{\varepsilon} \bar{U}_{\varepsilon}^{\prime}=-\lambda \bar{\gamma}_{\varepsilon}\left[\left[I-\lambda \overline{\mathcal{L}}_{\varepsilon}^{\text {qinv }}\right]^{-1} \overline{\mathcal{L}}_{\varepsilon}^{\text {qinv }}\left[\bar{U}_{\varepsilon}^{\prime}\right]\right] .
$$

On account of (6.14) we hence obtain the estimate

$$
\left|\bar{\gamma}_{\varepsilon} \bar{U}_{\varepsilon}^{\prime}\right| \leq C_{1}^{\prime}|\lambda| \leq C_{1}^{\prime} \lambda_{I}
$$

for some $C_{1}^{\prime}>0$. However, Lemma 6.4 shows that the left-hand side remains bounded away from zero, which yields the desired contradiction after restricting the size of $\lambda_{I}$.

Lemma 6.7. Assume that (HN1), (HN2), (HW1), (HW2), (HS1), and (HS2) are satisfied. There exist constants $\lambda_{I I}>0$ and $\varepsilon_{I I}>0$ so that the operator $\overline{\mathcal{L}}_{\varepsilon, \lambda}: \mathbf{H}^{1} \rightarrow$ $\mathbf{L}^{2}$ is injective for all $\lambda \in \mathcal{R}$ with $\operatorname{Re} \lambda \geq \lambda_{I I}$ and $0<\varepsilon<\varepsilon_{I I}$.

Proof. The identity $\overline{\mathcal{L}}_{\varepsilon, \lambda} \Phi=0$ implies that

$$
c_{\varepsilon} \Phi^{\prime}=\mathcal{M}_{1 / \varepsilon^{2}}^{1} J_{\mathrm{mix}} \Phi+D F\left(\bar{U}_{\varepsilon}\right) \Phi-\lambda \Phi .
$$

Taking the inner product with $\mathcal{M}_{\varepsilon^{2}}^{1,2} \Phi$, we may use Lemma 4.11 to obtain

$$
\begin{aligned}
0 & \leq-\operatorname{Re}\left\langle J_{\text {mix }} \Phi, \Phi\right\rangle_{\mathbf{L}^{2}} \\
& =\operatorname{Re}\left\langle D F\left(\bar{U}_{\varepsilon}\right) \Phi, \mathcal{M}_{\varepsilon^{2}}^{1,2} \Phi\right\rangle_{\mathbf{L}^{2}}-\operatorname{Re} \lambda\left\|\mathcal{M}_{\varepsilon}^{1,2} \Phi\right\|_{\mathbf{L}^{2}} \\
& \leq\left(K_{F}-\operatorname{Re} \lambda\right)\left\|\mathcal{M}_{\varepsilon}^{1,2} \Phi\right\|_{\mathbf{L}^{2}} .
\end{aligned}
$$

For $\operatorname{Re} \lambda \geq K_{F}$ this hence implies $\Phi=0$, as desired.

Proof of Proposition 6.1. On account of Lemmas 6.3, and 6.5-6.7, it remains to consider the set

$$
M=\left\{\lambda \in \mathcal{R}:|\lambda| \geq \lambda_{I}, \operatorname{Re} \lambda \leq \lambda_{I I}\right\} .
$$

Since this set satisfies $\left(\mathrm{hM}_{\lambda_{0}}\right)$, we can apply Proposition 4.2 to show that for each sufficiently small $\varepsilon>0$, the operators $\overline{\mathcal{L}}_{\varepsilon, \lambda}$ are invertible for all $\lambda \in M$.

\section{REFERENCES}

[1] P. W. Bates, X. Chen, And A. ChmaJ, Traveling waves of bistable dynamics on a lattice, SIAM J. Math. Anal., 35 (2003), pp. 520-546.

[2] K. Bhattacharya, Microstructure of Martensite: Why it Forms and How it Gives Rise to the Shape-Memory Effect, Vol. 2, Oxford University Press, Oxford, 2003. 
[3] P. C. Bressloff, Spatiotemporal dynamics of continuum neural fields, J. Phys. A, 45 (2011).

[4] P. C. Bressloff, Waves in Neural Media: From Single Neurons to Neural Fields, Lect. Notes Math. Model. Life Sci., Springer, New York, 2014.

[5] M. Brucal-Hallare and E. S. VAN Vleck, Traveling wavefronts in an antidiffusion lattice Nagumo model, SIAM J. Appl. Dyn. Syst., 10 (2011), pp. 921-959.

[6] J. W. CAHN AND A. NoviCK-CoHEN, Evolution equations for phase separation and ordering in binary alloys, J. Stat. Phys., 76 (1994), pp. 877-909.

[7] J. W. CAHN AND E. S. VAN VleCK, On the co-existence and stability of trijunctions and quadrijunctions in a simple model, Acta Mater., 47 (1999), pp. 4627-4639.

[8] G. Carpenter, A geometric approach to singular perturbation problems with applications to nerve impulse equations, J. Differential Equations, 23 (1977), pp. 335-367.

[9] P. Carter, B. DE RiJk, AND B. SAndstede, Stability of traveling pulses with oscillatory tails in the FitzHugh-Nagumo system, J. Nonlinear Sci., 26 (2016), pp. 1369-1444.

[10] P. Carter and B. Sandstede, Fast pulses with oscillatory tails in the FitzHugh-Nagumo system, SIAM J. Math. Anal., 47 (2015), pp. 3393-3441.

[11] C. Chen And Y. ChoI, Traveling pulse solutions to FitzHugh-Nagumo equations, Calc. Var. Partial Differential Equations, 54 (2015), pp. 1-45.

[12] X. Chen, J. S. Guo, And C. C. Wu, Traveling waves in discrete periodic media for bistable dynamics, Arch. Ration. Mech. Anal., 189 (2008), pp. 189-236.

[13] P. Connwell, Opening the Maslov Box for Traveling Waves in Skew-Gradient Systems, preprint, arXiv:1709.01908, 2017.

[14] P. Cornwell And C. K. R. T. Jones, On the existence and stability of fast traveling waves in a doubly diffusive FitzHugh-Nagumo system, SIAM J. Appl. Dyn. Syst., 17 (2018), pp. $754-787$.

[15] E. D'Este, D. Kamin, F. Göttfert, A. El-Hady, and S. E. Hell, STED nanoscopy reveals the ubiquity of subcortical cytoskeleton periodicity in living neurons, Cell Rep., 10 (2015), pp. $1246-1251$.

[16] E. D’Este, D. Kamin, C. Velte, F. Göttfert, M. Simons, and S. E. Hell, Subcortical cytoskeleton periodicity throughout the nervous system, Sci. Rep., 6 (2016), 22741.

[17] J. J. Duistermant And J. A. Kolk, Multidimensional Real Analysis I: Differentiation, Cambridge Stud. Adv. Math. 86, Cambridge University Press, Cambridge, 2004.

[18] T. E. FAVER, Nanopteron-sTegoton Traveling Waves in Spring Dimer Fermi-Pasta-UlamTsingou Lattices, preprint, arXiv:1710.07376, 2017.

[19] T. E. Faver And J. D. Wright, Exact Diatomic Fermi-Pasta-Ulam-Tsingou Solitary Waves with Optical Band Ripples at Infinity, SIAM J. Math. Anal., 50 (2018), pp. 182-250.

[20] G. FAye AND A. SCheEl, Existence of pulses in excitable media with nonlocal coupling, Adv. Math., 270 (2015), pp. 400-456.

[21] R. FitzHugh, Impulses and physiological states in theoretical models of nerve membrane, Biophys. J., 1 (1966), pp. 445-466.

[22] R. FitzHugh, Mathematical models of excitation and propagation in nerve, in Biological Engineering, McGraw Hill, New York, 1969.

[23] R. FitzHugh, Motion picture of nerve impulse propagation using computer animation, J. Appl. Physiology, 25 (1968), pp. 628-630.

[24] J. S. GuO AND C.-C. Wu, Uniqueness and stability of traveling waves for periodic monostable lattice dynamical system, J. Differential Equations, 246 (2009), pp. 489-525.

[25] S. Hastings, On travelling wave solutions of the Hodgkin-Huxley equations, Arch. Ration. Mech. Anal., 60 (1976), pp. 229-257.

[26] A. L. Hodgkin And A. F. Huxley, A quantitative description of membrane current and its application to conduction and excitation in nerve, J. Physiology, 117 (1952).

[27] A. Hoffman AND J. D. WRIGHT, Nanopteron solutions of diatomic Fermi-Pasta-UlamTsingou lattices with small mass-ratio, Phys. D, 358 (2017), pp. 33-59.

[28] H. J. Hupkes, L. Morelli, And P. Stehlik, Bichromatic Travelling Waves for Lattice Nagumo Equations, preprint, arXiv:1805.10977, 2018.

[29] H. J. Hupkes And B. SAndstede, Travelling pulse solutions for the discrete FitzHugh-Nagumo system, SIAM J. Appl. Dyn. Syst., 9 (2010), pp. 827-882.

[30] H. J. Hupkes And B. SAndstede, Stability of pulse solutions for the discrete FitzHugh-Nagumo system, Trans. Amer. Math. Soc., 365 (2013), pp. 251-301.

[31] H. J. Hupkes and E. S. Van Vleck, Travelling Waves for Adaptive Grid Discretizations of Reaction-Diffusion Systems, preprint.

[32] H. J. Hupkes And E. S. VAN VleCK, Travelling waves for complete discretizations of reaction diffusion systems, J. Dynam. Differential Equations, 28 (2016), pp. 955-1006.

[33] C. K. R. T. Jones, Stability of the travelling wave solutions of the FitzHugh-Nagumo system, Trans. Amer. Math. Soc, 286 (1984), pp. 431-469.

Copyright (c) by SIAM. Unauthorized reproduction of this article is prohibited. 
[34] C. K. R. T. Jones, N. Kopell, And R. Langer, Construction of the FitzHugh-Nagumo pulse using differential forms, in Patterns and Dynamics in Reactive Media, H. Swinney, G. Aris, and D. G. Aronson, eds., IMA Vol. Math. Appl. 37, Springer, New York, 1991, pp. 101-116.

[35] J. Keener And J. Sneed, Mathematical Physiology, Springer, New York, 1998.

[36] M. Krupa, B. Sandstede, and P. Szmolyan, Fast and slow waves in the FitzHugh-Nagumo equation, J. Differential Equations, 133 (1997), pp. 49-97.

[37] R. S. LiLliE, Factors affecting transmission and recovery in the passive iron nerve model, J. General Physiology, 7 (1925), pp. 473-507.

[38] J. Mallet-Paret, Spatial patterns, spatial chaos and traveling waves in lattice differential equations, in Stochastic and Spatial Structures of Dynamical Systems, Royal Netherlands Academy of Sciences, Amsterdam, 1996, pp. 105-129.

[39] J. Mallet-PAREt, The Fredholm alternative for functional differential equations of mixed type, J. Dynam. Differential Equations, 11 (1999), pp. 1-48.

[40] D. J. Pinto and G. B. Ermentrout, Spatially structured activity in synaptically coupled neuronal networks: 1. Traveling fronts and pulses, SIAM J. Appl. Math., 62 (2001), pp. 206225.

[41] L. A. Ranvier, Lećons sur l'Histologie du Système Nerveux, par M. L. Ranvier, recueillies par M. Ed. Weber, F. Savy, Paris, 1878.

[42] W. M. Schouten-Straatman and H. J. Hupkes, Travelling Pulse Solutions for Completely Discrete FitzHugh-Nagumo Type Equations with Infinite-Range Interactions, in preparation.

[43] W. M. Schouten-Straatman and H. J. Hupkes, Nonlinear Stability of Pulse Solutions for the Discrete Fitzhugh-Nagumo Equation with Infinite-Range Interactions, http://pub.math. leidenuniv.nl/ hupkeshj/fhninfr.pdf, 2017.

[44] W. M. Schouten-Straatman and H. J. Hupkes, Nonlinear stability of pulse solutions for the discrete Fitzhugh-Nagumo equation with infinite-range interactions, Discrete Contin. Dyn. Syst. Ser. A, 39 (2019), pp. 5017-5083.

[45] J. Sneyd, Tutorials in Mathematical Biosciences II, Mathematical Modeling of Calcium Dynamics and Signal Transduction, Lecture Notes in Math. 187, Springer, New York, 2005.

[46] A. K. Tagantsev, L. E. Cross, and J. Fousek, Domains in Ferroic Crystals and Thin Films, Springer, New York, 2010.

[47] A. Vainchtein And E. S. VAn Vleck, Nucleation and propagation of phase mixtures in a bistable chain, Phys. Rev. B, 79 (2009), 144123.

[48] A. Vainchtein, E. S. VAn Vleck, And A. Zhang, Propagation of periodic patterns in a discrete system with competing interactions, SIAM J. Appl. Dyn. Syst., 14 (2015), pp. 523-555.

[49] K. Xu, G. Zhong, And X. Zhuang, Actin, spectrin, and associated proteins form a periodic cytoskeletal structure in axons, Science, 339 (2013), pp. 452-456.

Copyright (c) by SIAM. Unauthorized reproduction of this article is prohibited. 